OPEN ACCESS

Edited by:

Simone Rossi,

University of Siena, Italy

Reviewed by:

Victor Manuel Pulgar,

Wake Forest School of Medicine,

United States

Yun Qian,

Shanghai Jiao Tong University, China

Maria Egle De Stefano,

Sapienza University of Rome, Italy

Stefan Reuss,

Johannes Gutenberg University

Mainz, Germany

*Correspondence:

Yusuf Ozgur Cakmak

yusuf.cakmak@otago.ac.nz

Specialty section:

This article was submitted to

Neural Technology,

a section of the journal

Frontiers in Neuroscience

Received: 24 September 2019

Accepted: 09 March 2020

Published: 02 April 2020

Citation:

Lumsden SC, Clarkson AN and

Cakmak YO (2020) Neuromodulation

of the Pineal Gland via Electrical

Stimulation of Its Sympathetic

Innervation Pathway.

Front. Neurosci. 14:264.

doi: 10.3389/fnins.2020.00264

\section{Neuromodulation of the Pineal Gland via Electrical Stimulation of Its Sympathetic Innervation Pathway}

\author{
Susannah C. Lumsden ${ }^{1,2}$, Andrew N. Clarkson ${ }^{1,3,4}$ and Yusuf Ozgur Cakmak $k^{1,2,4,5 *}$ \\ 1 Department of Anatomy, University of Otago, Dunedin, New Zealand, ${ }^{2}$ Brain Health Research Centre, Dunedin, New \\ Zealand, ${ }^{3}$ Brain Research New Zealand, Dunedin, New Zealand, ${ }^{4}$ Medical Technologies Centre of Research Excellence, \\ Auckland, New Zealand, ${ }^{5}$ Centre for Health Systems and Technology, Dunedin, New Zealand
}

Stimulation of the pineal gland via its sympathetic innervation pathway results in the production of $\mathrm{N}$-acetylserotonin and melatonin. Melatonin has many therapeutic roles and is heavily implicated in the regulation of the sleep-wake cycle. In addition, $\mathrm{N}$-acetylserotonin has recently been reported to promote neurogenesis in the brain. Upregulation of these indoleamines is possible via neuromodulation of the pineal gland. This is achieved by electrical stimulation of structures or fibres in the pineal gland sympathetic innervation pathway. Many studies have performed such pineal neuromodulation using both invasive and non-invasive methods. However, the effects of various experimental variables and stimulation paradigms has not yet been reviewed and evaluated. This review summarises these studies and presents the optimal experimental protocols and stimulation parameters necessary for maximal upregulation of melatonin metabolic output.

Keywords: pineal, stimulation, neuromodulation, SCG, NAS, melatonin, AANAT, sympathetic

\section{INTRODUCTION}

The pineal gland is an azygous, endocrine gland located in the midline of the brain. In humans, it is one solid structure situated deep within the brain between the habenular and posterior commissures, directly posterior to the third ventricle. In rodents, the gland is comprised of superficial, deep and stalk components. The superficial gland is found on the surface of the brain - anterior to the cerebellum and directly beneath the confluens sinuum. The pineal gland is comprised of a variety of cell types: pinealocytes, microglia, astrocytes, vascular and leptomeningeal cells, and endothelial cells. It is possible to distinguish between pinealocytes and other cell types using electrophysiological characteristics specific to each type. For example, astrocytes do not exhibit a biphasic positive-negative waveform composed of an initial segment-soma dendritic inflexion, whereas pinealocytes do. Pinealocytes are distributed uniformly throughout the gland and are predominantly responsible for the synthesis and secretion of melatonin in response to environmental lighting changes (Mays et al., 2018). Melatonin is considered the chemical expression of darkness and in the absence of light is secreted in response to signals from the suprachiasmatic nucleus (SCN). Melatonin is a systemically ubiquitous molecule and is also secreted from extrapineal sites including the Harderian gland, retina, and GI tract (for review see: Huether, 1993). 
For melatonin synthesis (Figure 1), tryptophan is uptaken into the pinealocyte from the blood and converted into 5hydroxytryptophan (5-HTP) via tryptophan-5-hydroxylase. 5HTP is then converted into serotonin by 5-HTP decarboxylase, before serotonin is converted to $\mathrm{N}$-acetylserotonin (NAS) by the enzyme aralkylamine $\mathrm{N}$-acetyltransferase (AANAT). The enzyme, hydroxyindole-O-methyltransferase (HIOMT) then converts NAS into melatonin, which is secreted directly into the bloodstream or cerebrospinal fluid (CSF) (Tan et al., 2018). Circulating norepinephrine (NE) is unable to contribute to pineal innervation. This is because the postganglionic sympathetic nerves actively take up circulating catecholamines to prevent persistent activation of the pineal, and also maintain the gland's circadian rhythmicity (Wetterberg, 1979; Reiter, 1990).

The anatomical pathway providing sympathetic input to the pineal gland is the in-road for pineal modulation. The polysynaptic innervation pathway of the gland (Figure 2) is as follows: an absence of light is detected by the retina and this information is transmitted via the retinohypothalamic tract (RHT) to the master pacemaker of the brain - the suprachiasmatic nucleus (SCN) (Hendrickson et al., 1972; Moore and Lenn, 1972; Moore, 1973). Information from the SCN is then transmitted to the paraventricular nucleus (PVN) (Vrang et al., 1995; Kalsbeek et al., 2000; Munch et al., 2002) whose fibres descend to connect with the intermediolateral column of the thoracic spinal cord (IML). Projections from the IML then ascend to the superior cervical ganglia (SCG) and then SCG postganglionic sympathetic fibres ascend through the
Tryptophan

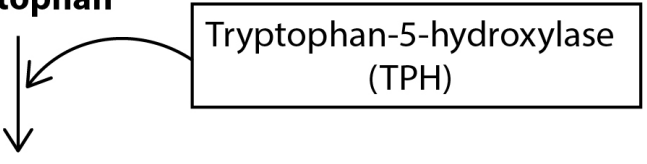

\section{5-hydroxytryptophan}

(5-HTP)

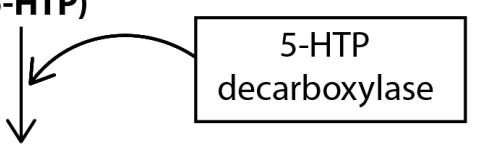

Serotonin

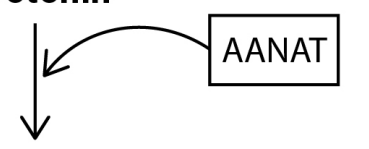

NAS

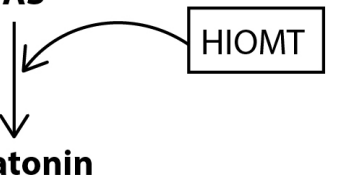

FIGURE 1 | Biosynthesis of melatonin. Tryptophan is converted to 5-hydroxytryptophan (5-HTP) by the enzyme tryptophan-5-hydroxylase (TPH). 5-HTP is then converted to serotonin via the enzyme 5-HTP decarboxylase. Serotonin is subsequently converted to NAS and then melatonin via the enzymes AANAT and HIOMT, respectively. internal carotid canal (Bowers et al., 1984b), accompanied by the internal carotid artery and innervate pinealocytes (Bargmann, 1943; Kappers, 1960a). Postganglionic sympathetic fibres initially contact the pineal from the dorso-posterior aspect (Kappers, 1960b; Bowers et al., 1984b). These sympathetic fibres are generally arranged into two distinct bundles known as "nervi conarii," however, sometimes they become fused and reach the gland as one bundle (le Gros Clark, 1940; Kappers, 1960b). The nervi conarii form a plexus over the entirety of the pineal gland, with nerve fascicles originating from both left and right SCG intermingling on its surface (Bowers et al., 1984b). Each innervating nervi conarii provides equal innervation to both the ipsilateral and contralateral side of the gland (Lingappa and Zigmond, 1987). These fibres' terminals often end in perivascular spaces (Huang and Lin, 1984) where they release NE onto pinealocytes during the night to stimulate melatonin synthesis.

Inhibitory and excitatory responses have been recorded from pinealocytes. There may be specific groups of pinealocytes that become excited or inhibited depending upon the source of innervation i.e., whether innervated by fibres from the left, right, or both SCG (Reuss et al., 1985b). Regulation of pineal electrical responses might be also mediated by pinealocytes themselves, with the excitation of one pinealocyte causing the inhibition of another (Reuss, 1986). Yet, whilst it is generally accepted that hyperpolarisation of pinealocytes leads to melatonin synthesis (Sakai and Marks, 1972; Parfitt et al., 1975; Freschi and Parfitt, 1986), exactly how different types of electrical response regulate this process is unknown. One possible mechanism could be pinealocytes engaging in selfregulation through glutamate release following hyperpolarisation of the cell membrane. Glutamate may then act in an autocrine and/or paracrine manner via mGluR3 receptors which have been demonstrated on the pinealocyte cell membrane (Yamada et al., 1996a,b; Yatsushiro et al., 2000). The mGluR3 receptors are negatively coupled to adenylate cyclase (AC) through an inhibitory G-protein. This decreases AANAT activity which converts NAS into melatonin, thus decreasing melatonin synthesis (Yamada et al., 1998). Alternatively, pinealocytes may be involved in the formation of a tripartite synapse via the inclusion of astrocytes in the communication between the postganglionic sympathetic terminals and the pinealocyte membrane (Villela et al., 2013). Glutamate release may trigger an increase in astrocyte intracellular calcium levels $\left(\left[\mathrm{Ca}^{2+}\right]_{\mathrm{i}}\right)$ and activation of nuclear factor kappa-light-chain-enhancer of activated $\beta$-cells (Villela et al., 2013). Astrocytes may then release particular gliotransmitters (e.g., tumour necrosis factor alpha $\mathrm{TNF} \alpha$ ) that act on receptors on the pinealocyte membrane, either alone or in conjunction with glutamate (Villela et al., 2013). These gliotransmitters may provide negative feedback to the presynaptic sympathetic terminal, preventing further release of NE (Parpura et al., 1994; Villela et al., 2013), or elicit inhibitory or excitatory responses in the post-synaptic pinealocyte membrane (Hassinger et al., 1995; Villela et al., 2013). Moreover, the number of AMPA receptors in the pinealocyte membrane may be upregulated through TNF $\alpha$ (Villela et al., 2013) - which may be the receptors through which $\mathrm{TNF} \alpha$ and glutamate exert their effects. TNF $\alpha$ has also been shown to decrease NAS and serotonin 


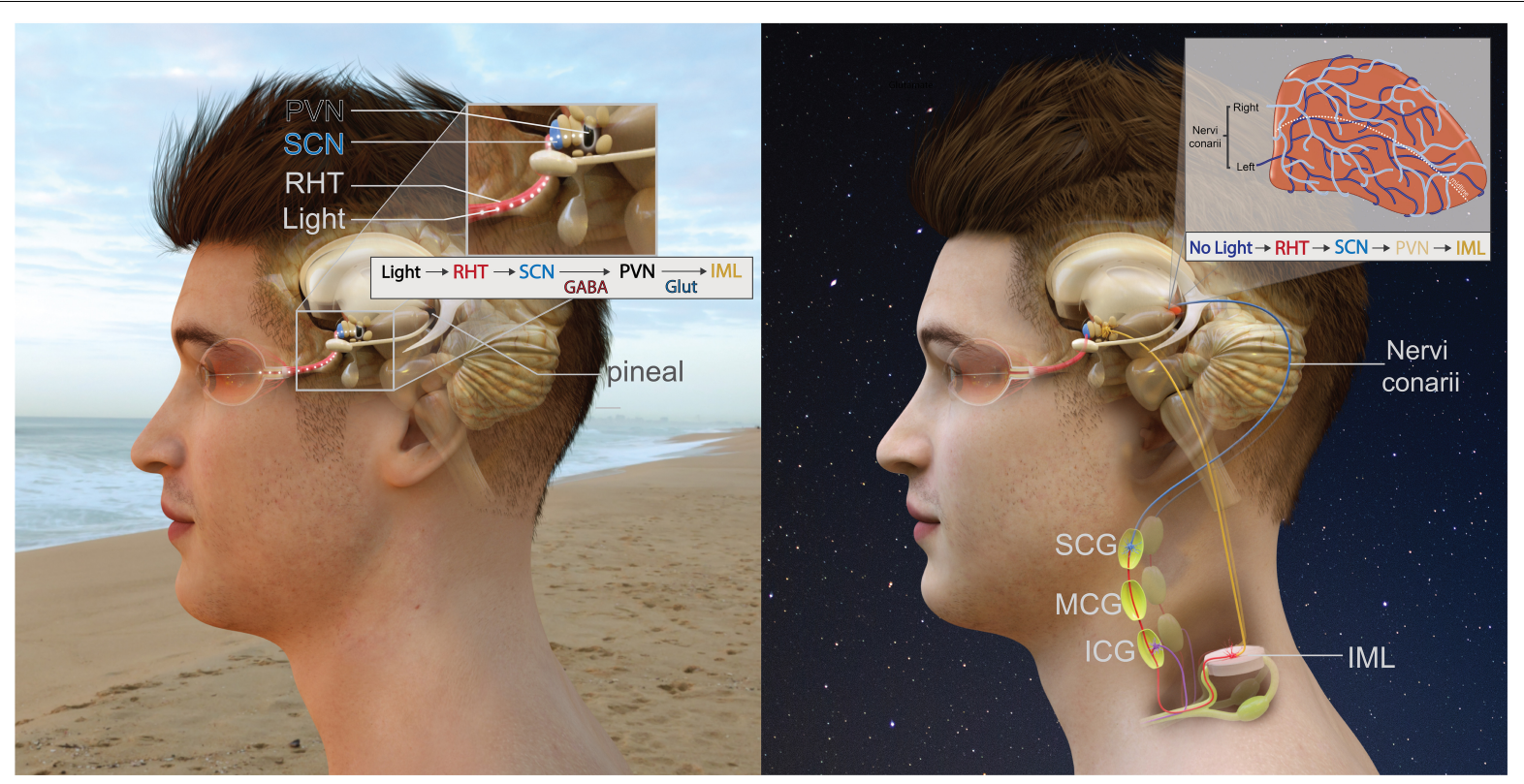

FIGURE 2 | Innervation of the pineal gland. The RHT which projects to the SCN followed by the PVN. The pineal pathway then descends down the spinal cord to the IML. Preganglionic sympathetic fibres ascend, pass through the inferior and middle cervical ganglia before terminating on the SCG. Postganglionic sympathetic fibres then ascend and innervate the pineal gland. (A-left) During the day, this pathway is inhibited due to light. (B-right) During the night, an absence of light activates this pathway and the pineal gland receives sympathetic input.

levels as well as AANAT mRNA expression (Tsai et al., 2001; Fernandes et al., 2006). Melatonin levels may be regulated via one, or a combination of the above mechanisms (see Figure 3).

The response of pinealocytes to adrenergic stimulation is complex, with an initial transient rapid hyperpolarisation followed by a sustained depolarisation (Zemkova et al., 2011). This hyperpolarisation is due in part to $\mathrm{K}^{+}$efflux from largeconductance $\mathrm{Ca}^{2+-}$ activated $\mathrm{K}^{+}$channels $\left(\mathrm{BK}_{\mathrm{Ca} 2+}\right.$ ) (Cena et al., 1991). An increase in $\left[\mathrm{Ca}^{2+}\right]_{\mathrm{i}}$ and cyclic adenosine monophosphate (cAMP) is necessary for the opening of these channels (Cena et al., 1991). This triggers a myriad of intracellular molecular cascades that eventually results in melatonin synthesis (see Figure 3 and Supplementary Material for further details). However, it seems that the spontaneous electrical activity of all pinealocytes is not identical. Some researchers have grouped pinealocytes accordingly into 'clusters' of regularly firing cells (REG) and rhythmically firing cells (RHY), the latter forming the minority (Schenda and Vollrath, 1997; Figure 4). Each cluster is composed of 3-5 of one type and surrounded by 'silent' cells which exhibit no spontaneous firing (Schenda and Vollrath, 1997). The RHY clusters appear to interact with one another synaptically, with the firing of one cluster being linked to the firing of another (Schenda and Vollrath, 1999). This intrapineal network linkage may contribute to the regulation of extracellular substances known to stimulate or inhibit melatonin synthesis such as NE and acetylcholine, respectively (Yamada et al., 1996a,b; Schenda and Vollrath, 1999). Others have also suggested classifying pinealocytes into different categories due to morphological heterogeneity (Calvo and Boya, 1984; Al-Hussain, 2006), or differences in the quantitative presence of the HIOMT enzyme (Rath et al., 2016). However, at present there exists no definitive clarification for how such heterogeneity relates to melatonin metabolism.

The sympathetic innervation pathway of the pineal involving the PVN, SCN, and SCG is generally accepted to provide the only functional input to the gland. The SCG together with the middle and inferior cervical ganglia comprise the cervical sympathetic trunks (CSTs). Stimulation of the distal portion of the CST closest to the SCG invokes a significant increase in the rate of glucose metabolism in both the ipsilateral SCG and the pineal, but not elsewhere in the central nervous system (CNS) (Ito et al., 1988) reflecting the likely exclusivity of this pathway. However, other structures may also innervate the gland such as: the trigeminal ganglion (Yamamoto et al., 1983; Shiotani et al., 1986; Moller et al., 1993; Matsushima et al., 1994; Matsuura et al., 1994; Reuss, 1999), the lateral geniculate nucleus (Korf and Wagner, 1980; Mikkelsen and Moller, 1990), lateral hypothalamus (Fink-Jensen and Møller, 1990), the dorsal raphe nucleus (Leander et al., 1998; Moller and Hay-Schmidt, 1998), and fibres such as the habenular and posterior commissures (Moller, 1978; Moller and Korf, 1983; Reuss and Moller, 1986; Larsen et al., 1991), and the greater petrosal nerve (Kenny, 1967; Romijn, 1975). Wurtman et al. (1963) proposed that such pathways are crucial for providing information regarding chronic changes in lighting conditions i.e., seasonal changes. By contrast, Schapiro and Salas (1971) argue they provide an alternative sympathetic pathway, separate from the SCG, supplying the pineal with information regarding acute changes to photic stimuli. With regards to the commissural inputs, the linkage between the commissures and pineal gland are not surprising as they are topographically linked during 


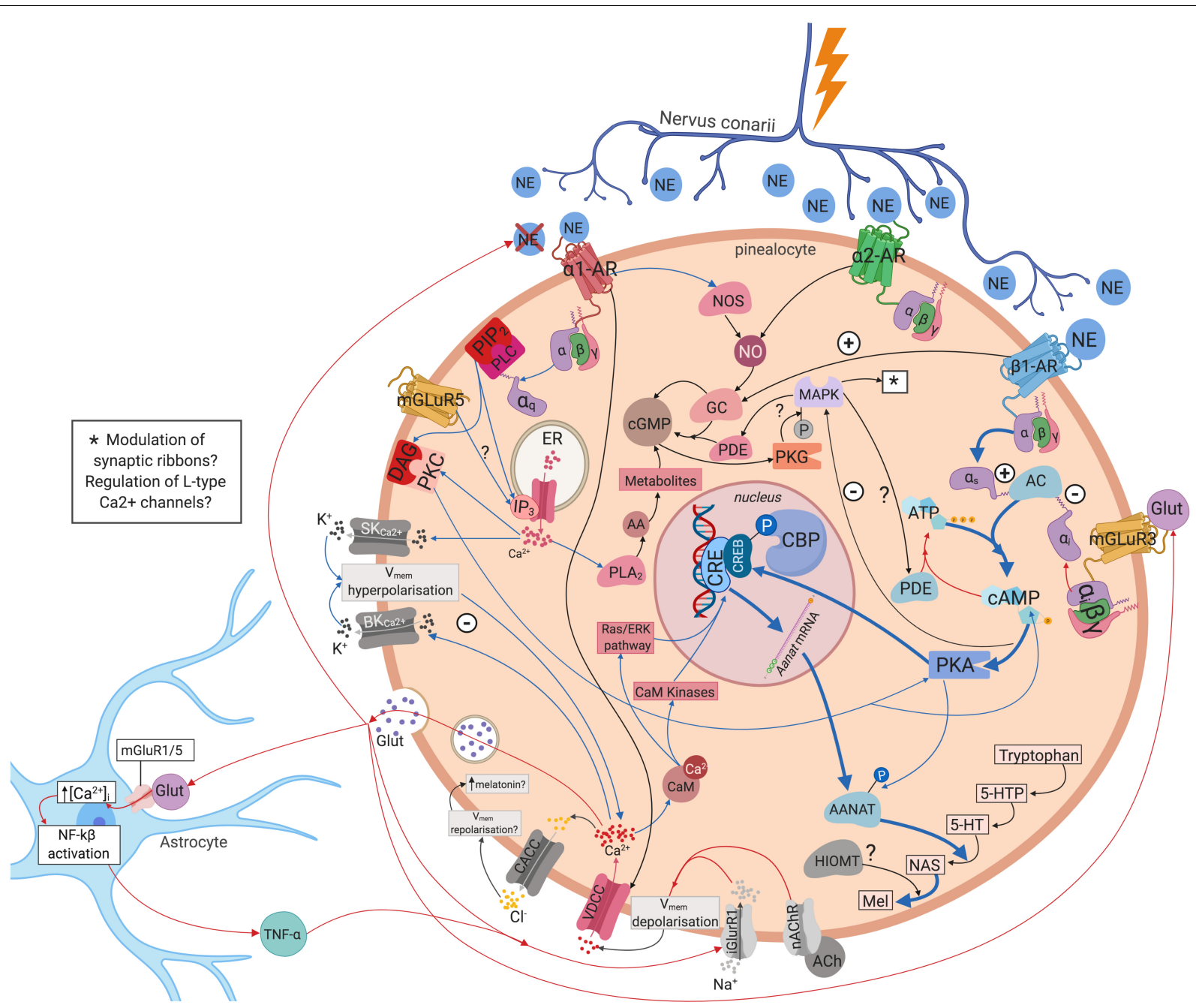

FIGURE 3 | Intra- and intercellular modulation of melatonin synthesis. NE binds to the $\alpha_{1}, \alpha_{2}$, and $\beta_{1}$ adrenergic receptors on pinealocytes triggering a complex myriad of intracellular molecular cascades that eventually regulate melatonin synthesis. Pinealocytes may engage in autocrine and/or paracrine regulation via glutamate release via mGluR3. Pinealocytes may also form a tripartite synapse with astrocytes and postganglionic sympathetic terminals. Blue arrows, upregulating melatonin synthesis; red arrows, downregulating melatonin synthesis; black arrows, mixed or unclear effects on melatonin synthesis. 5-HT, 5-hydroxytryptamine; 5-HTP, 5-hydroxytryptophan; AA, arachidonic acid; AANAT, aralkylamine N-acetyltransferase; AC, adenylate cyclase; AMPA,

$\alpha$-amino-3-hydroxy-5-methyl-4-isoxazolepropionic acid receptor; ATP, adenosine triphosphate; $\alpha_{1}$-AR, $a_{1}$-adrenoreceptor; $a_{2}$-AR, $a_{2}$-adrenoreceptor; BK $\mathrm{Ca}_{2}+$, large-conductance $\mathrm{Ca}^{2+-}$ activated $\mathrm{K}^{+}$channel; $\mathrm{CACC}, \mathrm{Ca}^{2+}$ activated $\mathrm{Cl}^{-}$channels; $\mathrm{CaM}$, calcium binding protein calmodulin; cAMP, cyclic guanosine monophosphate; CBP, CREB-binding protein; CGMP, cyclic guanosine monophosphate; CRE, CAMP response element; CREB, cAMP response element binding protein; DAG, diacylglycerol; ER, endoplasmic reticulum; GC, guanylate cyclase; Glut, glutamate; iGluR1, ionotropic glutamate receptor 1; $I_{3}$, inositol trisphosphate; MAPK, mitogen-activated protein kinase MAPK; Mel, melatonin; mGluR3, metabotropic glutamate receptor 3; mGluR5, metabotropic glutamate receptor 5; nAChR, nicotinic acetylcholine receptor; NAS, N-acetylserotonin; NE, norepinephrine; NF-k $\beta$, nuclear factor kappa-light-chain-enhancer of activated $\beta$ cells; NO, nitric oxide; NOS, nitric oxide synthetase; PDE, phosphodiesterase; PKA, protein kinase A; PKC, protein kinase C; PKG, protein kinase G; PLA2, phospholipase $A_{2}$; SKCa ${ }^{2+}$, small-conductance $\mathrm{Ca}^{2+-}$ activated $\mathrm{K}^{+}$channel; TNF- $\alpha$, tumour necrosis factor $-\alpha$; VDCC, voltage-dependent $\mathrm{Ca}^{2+}$ channels; $\beta 1$-AR, $\beta 1$-adrenoreceptor.

ontological development. However, it may be that any projections from these structures to the pineal are aberrant and nonfunctional (le Gros Clark, 1940; Kappers, 1960b, 1965; Romijn, 1975). These projections remain a matter of great debate amongst researchers and further research is required to elucidate their exact relationship with the pineal gland.

Melatonin's therapeutic potential extends to a number of different mechanisms including: increasing neurogenesis in the hippocampus (Kim et al., 2004; Ramirez-Rodriguez et al., 2009;
Rennie et al., 2009; Sotthibundhu et al., 2010; Crupi et al., 2011) and the induction, regulation, and prolonging of sleep (for review see: Dawson and Encel, 1993). Reduced melatonin levels are linked with chronic sleep disturbances (Haimov et al., 1994; Garfinkel et al., 1995) and advanced age in humans (Waldhauser et al., 1988). These observations have led to the development of the "melatonin replacement" hypothesis, which posits "(1) the age-related decline in melatonin, in some way, contributes to insomnia; and (2) replacement with 


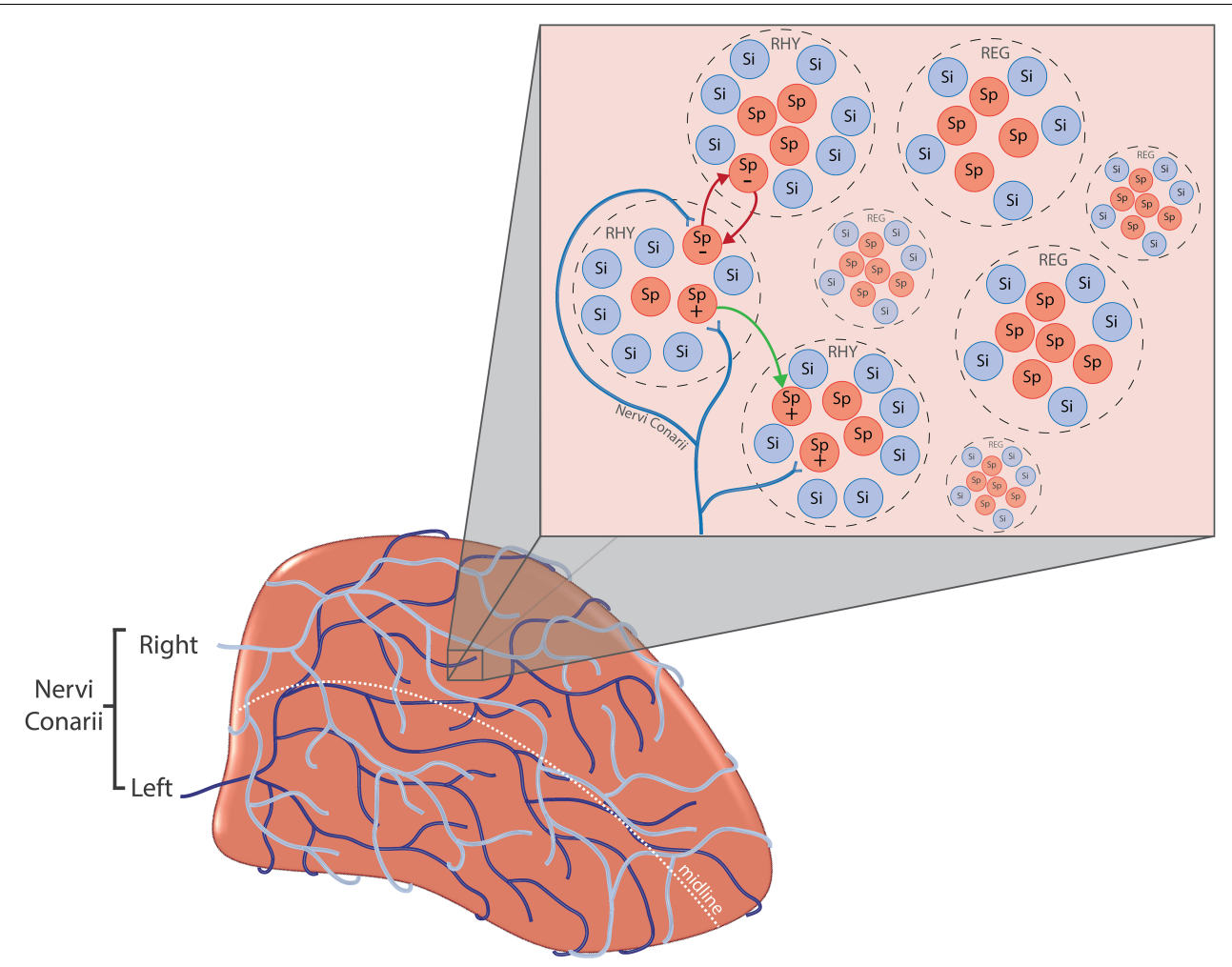

FIGURE 4 | (A-bottom left) Nervi conarii. The nervi conarii form an intermingling plexus of fibres over the pineal gland and release norepinephrine onto pinealocytes during the night to stimulate melatonin synthesis. (B-top right) Pinealocyte clusters. Spontaneously firing pinealocytes (Sp) are arranged into "clusters" of regularly firing cells (REG) and rhythmically firing cells (RHY). Clusters are surrounded by "silent" cells (Si) which exhibit no spontaneous firing. The firing of RHY cluster is synaptically linked to the firing of another (red arrows). The electrical response of pinealocytes is complex. Inhibitory and excitatory responses have been recorded from pinealocytes (+ and -). Specific groups of pinealocyte may become excited or inhibited depending upon the source of innervation i.e., left, right or both SCG. Regulation of pineal electrical responses might be also mediated by pinealocytes themselves, with the excitation of one pinealocyte causing the inhibition of another (green arrow).

high physiological doses of melatonin, will improve sleep" (Hughes et al., 1998). Consequently, oral administration of exogenous melatonin has been frequently utilised in clinical trials to upregulate melatonin levels and improve sleep. Oral administration of melatonin is temporally limited due to its quick absorption and relatively short plasma halflife (Waldhauser et al., 1984; Aldhous et al., 1985). Large doses of melatonin might maintain levels akin to endogenous levels, however, such large doses may place unnecessary strain on the liver, in addition to encouraging receptor desensitisation. Delivery of melatonin at regular intervals throughout the night to sustain endogenous levels has been suggested, however, this would require successive periods of waking, proving counterproductive in improving sleep. Further, modified release variants of melatonin have been investigated (Lemoine and Zisapel, 2012), but their efficacy is limited to elderly populations (Wade et al., 2010). Therefore, there exists demand for rapid-onset, long-lasting, non-pharmacological interventions addressing the problem of sleep disturbance via melatonin upregulation.

Neuromodulation is the "alteration of nerve activity through targeted delivery of a stimulus such as electrical stimulation..." (International Neuromodulation Society, 2018). Neuromodulation may be invasive or non-invasive. Invasive neuromodulation involves surgical intervention such as implantation of an electrical stimulation device directly into the body, which incurs risk and necessitates patient recovery time. Non-invasive techniques offer a safer alternative and include: stimulation of the scalp via transcranial electrical stimulation, transcutaneous electrical nerve stimulation (TENS) via stimulation of the skin that is not located on the scalp, and percutaneous electrical nerve stimulation (PENS) which may be considered a minimally invasive form of neuromodulation that involves stimulation delivered just under the skin e.g., electroacupuncture. Non-invasive techniques are often used to combat chronic neuropathic pain (Lefaucheur et al., 2001; Fregni et al., 2006a,b; Rossini et al., 2015), however, research indicates promising treatment avenues for other disorders such as tinnitus (Vanneste et al., 2010, 2013; Vanneste and De Ridder, 2011; De Ridder and Vanneste, 2012; Faber et al., 2012; Frank et al., 2012; Joos et al., 2014) and improving outcomes post-stroke (Fregni et al., 2005; Hummel et al., 2005, 2006; Khedr et al., 2005; Mansur et al., 2005; Takeuchi et al., 2005; Kim et al., 2006; Celnik et al., 2007, 2009). 
Electrical stimulation paradigms targeting the pineal sympathetic pathway have been trialled in animal studies and show pineal neuromodulation is possible. This review will attempt to summarise the pertinent findings of both invasive and non-invasive stimulatory studies in addition to the various experimental procedures used (summarised in Tables 1, 2). It is speculated that there exist differences of innervation mechanisms between mammals and birds. In birds, the melatonin synthesis pathway is not mediated via the sympathetic nervous system nor regulated by activation of $\beta$-adrenergic receptors. Therefore, this review will focus only on pineal stimulation studies involving mammals.

\section{STIMULATION OF STRUCTURES IN THE PINEAL GLAND SYMPATHETIC INNERVATION PATHWAY}

\section{Invasive Stimulation \\ Evoked Cell Potentials}

Experiments using electrical stimulation of the pineal sympathetic pathway have revealed significant heterogeneity pinealocyte response. Stimulation of the CSTs at $30 \mathrm{~Hz}$ (3 trains of $30 \mathrm{~Hz}$ for $1.25 \mathrm{~s}$ for $48 \mathrm{~s}$ per train) in rats was capable of evoking action potentials in the pineal gland (Brooks et al., 1975). In addition, use of $1 \mathrm{~Hz}$ frequency bilateral stimulation $(0.2 \mathrm{~ms}$ pulse, $0.1-0.6 \mathrm{~mA}$ ) of SCG postganglionic nerve fibres increased the firing rate of rat pineal cells (Reyes-Vazquez et al., 1986). Bowers and Zigmond (1982) bilaterally stimulated the CSTs in the rat at night-time and found a frequency dependent effect with temporal facilitation being observed in the postsynaptic potentials of the postganglionic fibres, with $10 \mathrm{~Hz}$ eliciting a greater effect compared with $1 \mathrm{~Hz}$ stimulation. The latter authors suggest that using higher frequencies allows for recruitment of a greater number of postganglionic fibres evoking action potentials that would otherwise remain "silent" if a continuous stimulus was used. These studies highlight that various frequencies are capable of influencing the pineal gland's sympathetic pathway.

An interaction between electrical pulse duration and current was discovered when a single electrical pulse of varying durations was delivered to the CSTs in order to examine the current required to produce an action potential in the internal carotid nerve (Bowers and Zigmond, 1982). It seems lower pulse durations $(<3 \mathrm{~ms})$ require a use of a greater current in order to elicit an action potential. Pulse durations of 3, 5, and $20 \mathrm{~m}$ appeared to require similarly low currents $(\sim 5 \mu \mathrm{A})$ in order to elicit an action potential. This indicates pulse duration may be manipulated in experiments requiring use of low currents in order to prevent hyperpolarisation of postganglionic cell membranes. However, replication of these results is necessary in order to confirm this strength-duration relationship.

Brooks et al. (1975) first demonstrated that bilateral stimulation of the CSTs evoked discharges in pinealocytes. The response prevalence of pinealocytes following invasive stimulation of the SCG either bilaterally or unilaterally has been investigated in a handful of studies (see Table 1). One such study found approximately half (44.7-55.8\%) of investigated cells elicited a significant electrical response following unilateral SCG stimulation during the daytime (Reuss et al., 1985b). This means that approximately half of cells investigated were unresponsive to innervation from just one ganglion. The authors note that the cells exhibited a preferential response for input from one ganglion. Whilst this could be attributed to factors such as submaximal stimulation, or investigated pinealocytes being too far away from innervating sympathetic fibres, this response rate is consistent with that previously reported (47\%) (Pazo and Gonzalez, 1991). However, lower (28\%) (Stehle et al., 1987), and much higher (62\%) (Patel and Demaine, 1990) response rates are also reported. Interestingly, the latter authors report no difference in response prevalence between unilateral and bilateral SCG stimulation. Another study encountered a similar response prevalence in $55 \%$ of pinealocytes following bilateral stimulation of the postganglionic fibres of the SCG (Reyes-Vazquez et al., 1986). However, following bilateral SCG stimulation, Reuss et al. (1985b) found a higher response rate (73.1\%). Interestingly, only $17.3 \%$ of these pinealocytes were responsive to input from both SCG, indicating only a small proportion of responding cells are influenced by contribution from both ganglia. This supports the notion that each ganglion provides innervation to the ipsilateral portion of the pineal gland, with only a small number of innervating fibres crossing the midline to innervate the contralateral portion of the gland simultaneously (RodriguezPerez, 1962). This view is now largely contested as recent research indicates equal innervation from both SCG and fibres intermingling to form a plexus over the gland (Bowers et al., 1984b; Lingappa and Zigmond, 1987).

The impact of unilateral vs. bilateral stimulation of the SCG remains poorly understood. Only two studies directly compare the response prevalence of pinealocytes following both unilateral and bilateral stimulation in rats and hamsters, respectively (Reuss et al., 1985b; Patel and Demaine, 1990). The contrasting response rates between unilateral and bilateral stimulation observed in these two studies may simply be due to interspecies differences between the hamster and the rat. In the rat, bilateral stimulation of the SCG evokes a greater response rate from pinealocytes (Reuss et al., 1985b) compared to unilateral stimulation, and the results from Pazo and Gonzalez (1991) seem to support this. The reason why Reyes-Vazquez et al. (1986) report a high response prevalence following unilateral stimulation similar to that reported for bilateral stimulation could be due to a number of reasons. Firstly, they stimulated the post-ganglionic fibres of the SCG whereas the other studies utilised stimulation of the SCG directly. This could have exaggerated the effect of stimulation delivered to the pineal. This could be because a purely excitatory response is being generated in the post-ganglionic fibres and this is translating to a greater response occurring in the pinealocytes. In contrast, when the SCG is stimulated directly, inhibitory signals being directed to the SCG could dampen the response of the SCG itself, which could in turn diminish any excitatory response being delivered to the post-ganglionic fibres, and therefore, the pinealocytes. Secondly, they utilised a stimulation frequency of $1 \mathrm{~Hz}$ whereas the other studies generally report use of higher frequencies. It has been observed that high and low 
Percentage of responsive cells that were... Percentage of total investigated cells that were...

\begin{tabular}{|c|c|c|c|c|c|c|c|c|c|c|c|c|c|c|c|c|c|}
\hline 要 & 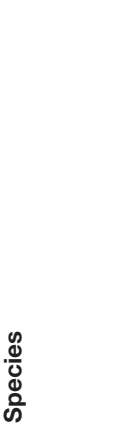 & 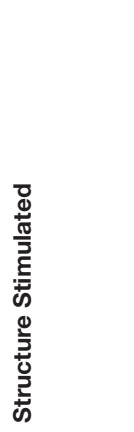 & 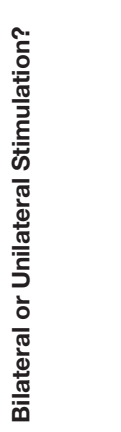 & 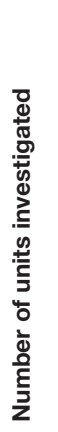 & 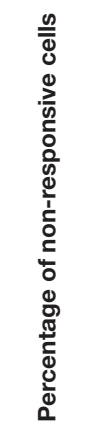 & 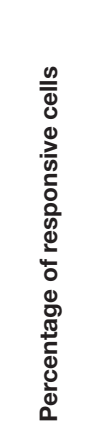 & 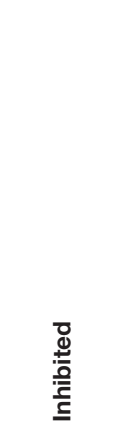 & 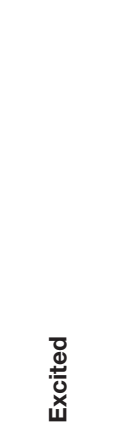 & $\begin{array}{l}\frac{0}{0} \\
\frac{\pi}{2} \\
\frac{0}{0} \\
\frac{10}{0}\end{array}$ & 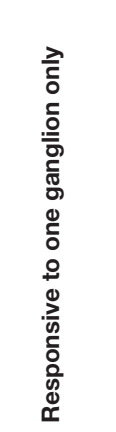 & 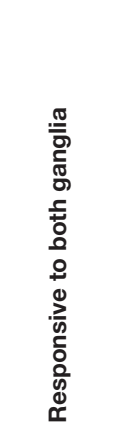 & 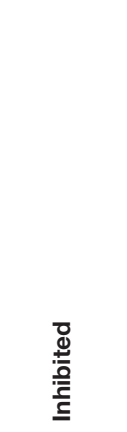 & 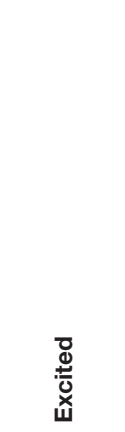 & $\begin{array}{l}\frac{0}{0} \\
\frac{0}{0} \\
\frac{0}{0} \\
\frac{0}{n}\end{array}$ & 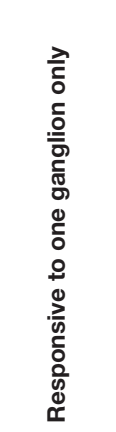 & 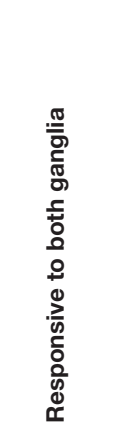 & 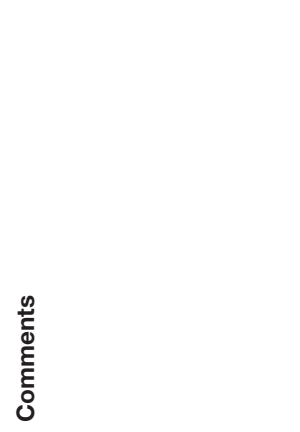 \\
\hline $\begin{array}{l}\text { Reuss } \quad F \\
\text { et al., } \\
1984\end{array}$ & Rat & $\begin{array}{l}\text { Habenular } \\
\text { nuclei }\end{array}$ & Bilateral & 42 & $\begin{array}{l}57.2 \% \\
(n=24)\end{array}$ & $\begin{array}{l}42.8 \% \\
(n=18)\end{array}$ & $\begin{array}{l}44.4 \% \\
(n=8)\end{array}$ & $\begin{array}{l}55.6 \% \\
(n=10)\end{array}$ & $\begin{array}{c}\text { Not } \\
\text { described }\end{array}$ & $\begin{array}{c}\text { Not } \\
\text { described }\end{array}$ & $\begin{array}{c}\text { Not } \\
\text { described }\end{array}$ & $\begin{array}{l}19 \% \\
(n=8)\end{array}$ & $\begin{array}{l}23.8 \% \\
(n=10)\end{array}$ & $\begin{array}{c}\text { Not } \\
\text { described }\end{array}$ & $\begin{array}{c}\text { Not } \\
\text { described }\end{array}$ & $\begin{array}{c}\text { Not } \\
\text { described }\end{array}$ & $\begin{array}{l}7 \text { silent units were found. } \\
\text { Confluens sinuum impaled } \\
\text { with electrode. }\end{array}$ \\
\hline \multirow[t]{2}{*}{$\begin{array}{l}\text { Reuss } \\
\text { et al., } \\
1985 b\end{array}$} & Rat & SCG & Unilateral & 94 & $\begin{array}{l}55.3 \% \\
(n=52)\end{array}$ & $\begin{array}{l}44.7 \% \\
(n=42)\end{array}$ & $\begin{array}{l}57.1 \% \\
(n=24)\end{array}$ & $\begin{array}{l}42.9 \% \\
(n=18)\end{array}$ & $\begin{array}{c}\text { Not } \\
\text { described }\end{array}$ & $\begin{array}{c}\text { Not } \\
\text { described }\end{array}$ & $\begin{array}{c}\text { Not } \\
\text { described }\end{array}$ & $\begin{array}{l}25.6 \% \\
(n=24)\end{array}$ & $\begin{array}{l}19.1 \% \\
(n=18)\end{array}$ & $\begin{array}{c}\text { Not } \\
\text { described }\end{array}$ & $\mathrm{N} / \mathrm{A}$ & N/A & $\begin{array}{l}13 \text { silent units were found. } \\
\text { Confluens sinuum impaled } \\
\text { with electrode. }\end{array}$ \\
\hline & & & Bilateral & 52 & $\begin{array}{l}26.9 \% \\
(n=14)\end{array}$ & $\begin{array}{l}73.1 \% \\
(n=38)\end{array}$ & $\begin{array}{c}\text { Not } \\
\text { described }\end{array}$ & $\begin{array}{c}\text { Not } \\
\text { described }\end{array}$ & $\begin{array}{c}\text { Not } \\
\text { described }\end{array}$ & $\begin{array}{l}76.3 \% \\
(n=29)\end{array}$ & $\begin{array}{l}23.7 \% \\
(n=9)\end{array}$ & $\begin{array}{c}\text { Not } \\
\text { described }\end{array}$ & $\begin{array}{c}\text { Not } \\
\text { described }\end{array}$ & $\begin{array}{c}\text { Not } \\
\text { described }\end{array}$ & $\begin{array}{l}55.8 \% \\
(n=29)\end{array}$ & $\begin{array}{l}17.3 \% \\
(n=9)^{\star}\end{array}$ & $\begin{array}{l}\text { Confluens sinuum impaled } \\
\text { with electrode. }\end{array}$ \\
\hline $\begin{array}{l}\text { Reyes- F } \\
\text { Vazquez } \\
\text { et al., } \\
1986\end{array}$ & Rat & SCG & Bilateral & 76 & $\begin{array}{l}44.7 \% \\
(n=34)\end{array}$ & $\begin{array}{l}55.3 \% \\
(n=42)\end{array}$ & $\begin{array}{c}\text { Not } \\
\text { described }\end{array}$ & $\begin{array}{l}55.3 \% \\
(n=42)\end{array}$ & $\begin{array}{c}\text { Not } \\
\text { described }\end{array}$ & $\begin{array}{c}\text { Not } \\
\text { described }\end{array}$ & $\begin{array}{c}\text { Not } \\
\text { described }\end{array}$ & $\begin{array}{c}\text { Not } \\
\text { described }\end{array}$ & $\begin{array}{l}55.3 \% \\
(n=42)\end{array}$ & $\begin{array}{c}\text { Not } \\
\text { described }\end{array}$ & $\begin{array}{c}\text { Not } \\
\text { described }\end{array}$ & $\begin{array}{c}\text { Not } \\
\text { described }\end{array}$ & $\begin{array}{l}6 \text { silent units were found. } \\
\text { Sagittal sinus ligated. }\end{array}$ \\
\hline \multirow{2}{*}{$\begin{array}{l}\text { Pazo } \\
\text { and } \\
\text { Gonzalez } \\
1991\end{array}$} & & SCG & Unilateral & 19 & $\begin{array}{c}53 \% \\
(n=10)\end{array}$ & $\begin{array}{l}47 \% \\
(n=9)\end{array}$ & $\begin{array}{l}56 \% \\
(n=5)\end{array}$ & $\begin{array}{c}33 \% \\
(n=3)\end{array}$ & $\begin{array}{l}11 \% \\
(n=1)\end{array}$ & $\mathrm{N} / \mathrm{A}$ & $\mathrm{N} / \mathrm{A}$ & $\begin{array}{l}26.3 \% \\
(n=5)\end{array}$ & $\begin{array}{l}26.3 \% \\
(n=5)\end{array}$ & $\begin{array}{l}5.3 \% \\
(n=1)\end{array}$ & $\mathrm{N} / \mathrm{A}$ & N/A & $\begin{array}{l}\text { In some animals, the } \\
\text { sagittal sinus was ligated }\end{array}$ \\
\hline & & $\mathrm{SCN}$ & Unilateral & 19 & $\begin{array}{c}47 \% \\
(n=9)\end{array}$ & $\begin{array}{c}53 \% \\
(n=10)\end{array}$ & $\begin{array}{l}50 \% \\
(n=5)\end{array}$ & $\begin{array}{l}30 \% \\
(n=3)\end{array}$ & $\begin{array}{l}20 \% \\
(n=2)\end{array}$ & $N / A$ & N/A & $\begin{array}{l}26.3 \% \\
(n=5)\end{array}$ & $\begin{array}{l}15.8 \% \\
(n=3)\end{array}$ & $\begin{array}{l}10.5 \% \\
(n=2)\end{array}$ & $N / A$ & N/A & \\
\hline $\begin{array}{l}\text { Stehle } \quad \vdash \\
\text { et al., } \\
1987\end{array}$ & Hamster & SCG & Unilateral & 48 & $\begin{array}{c}72 \% \\
(n=35)\end{array}$ & $\begin{array}{c}28 \% \\
(n=13)\end{array}$ & $\begin{array}{l}61.5 \% \\
(n=8)\end{array}$ & $\begin{array}{l}46.2 \% \\
(n=6)\end{array}$ & $\begin{array}{c}\text { Not } \\
\text { described }\end{array}$ & $\mathrm{N} / \mathrm{A}$ & N/A & $\begin{array}{l}16 \% \\
(n=8)\end{array}$ & $\begin{array}{l}12 \% \\
(n=6)\end{array}$ & $\begin{array}{c}\text { Not } \\
\text { described }\end{array}$ & $\mathrm{N} / \mathrm{A}$ & N/A & $\begin{array}{l}\text { Sagittal sinus ligated and } \\
\text { cut. See notes }^{\star \star}\end{array}$ \\
\hline $\begin{array}{l}\text { Patel } \vdash \\
\text { and } \\
\text { Demaine, } \\
1990\end{array}$ & Hamster & SCG & Both & 92 & $\begin{array}{c}38 \% \\
(n=35)\end{array}$ & $\begin{array}{c}62 \% \\
(n=57)\end{array}$ & $\begin{array}{l}36.8 \% \\
(n=21)\end{array}$ & $\begin{array}{l}36.8 \% \\
(n=21)\end{array}$ & $\begin{array}{l}15.8 \% \\
(n=9)^{\star \star \star}\end{array}$ & $\begin{array}{c}\text { Not } \\
\text { described }\end{array}$ & $\begin{array}{c}\text { Not } \\
\text { described }\end{array}$ & $\begin{array}{l}22.8 \% \\
(n=21)\end{array}$ & $\begin{array}{l}29.3 \% \\
(n=27)\end{array}$ & $\begin{array}{l}9.8 \% \\
(n=9)\end{array}$ & $\begin{array}{c}\text { Not } \\
\text { described }\end{array}$ & $\begin{array}{c}\text { Not } \\
\text { described }\end{array}$ & $\begin{array}{l}\text { No differences in form or } \\
\text { magnitude of response } \\
\text { found between unilateral vs } \\
\text { bilateral or right vs left SCG } \\
\text { stimulation. Confluens } \\
\text { sinuum impaled with } \\
\text { electrode. }\end{array}$ \\
\hline $\begin{array}{l}\text { Semm C } \\
\text { et al., } \\
1981\end{array}$ & Guinea-pig & $\begin{array}{l}\text { Lateral } \\
\text { habenular } \\
\text { nuclei }\end{array}$ & Bilateral & 128 & $\begin{array}{c}56 \% \\
(n=72)\end{array}$ & $\begin{array}{c}44 \% \\
(n=56)\end{array}$ & $\begin{array}{c}44 \% \\
(n=56)\end{array}$ & $\begin{array}{c}20 \% \\
(n=11)\end{array}$ & $\begin{array}{c}\text { Not } \\
\text { described }\end{array}$ & $\begin{array}{c}\text { Not } \\
\text { described }\end{array}$ & $\begin{array}{c}\text { Not } \\
\text { described }\end{array}$ & $\begin{array}{c}35 \% \\
(n=45)\end{array}$ & $\begin{array}{c}9 \% \\
(n=11)\end{array}$ & $\begin{array}{c}\text { Not } \\
\text { described }\end{array}$ & $\begin{array}{c}\text { Not } \\
\text { described }\end{array}$ & $\begin{array}{c}\text { Not } \\
\text { described }\end{array}$ & $\begin{array}{l}\text { No mention is made of the } \\
\text { confluens sinuum in the } \\
\text { methodology. }\end{array}$ \\
\hline
\end{tabular}

${ }^{*} 7.7 \%(n=4)$ of these cells showed different effects depending on whether left or right SCG was stimulated i.e., excitation or inhibition. Simultaneous stimulation showed no clear effects. **The number of units investigated in this study appears erroneous. This may possibly be due to a rounding error. ${ }^{* * * T h i s ~ c a t e g o r i s a t i o n ~ i n c l u d e s ~ a ~ p o p u l a t i o n ~ o f ~ c e l l s ~ w h i c h ~ e x h i b i t e d ~ i n c o n s i s t e n t ~ r e s p o n s e s ~ f o l l o w i n g ~ r e p e a t e d ~ s t i m u l a t i o n . ~}$ 
TABLE 2 | Summary of experimental procedures and stimulation parameters for reviewed studies concerning both invasive and non-invasive stimulation.

\begin{tabular}{|c|c|c|c|c|c|c|c|c|c|c|c|c|c|c|c|c|}
\hline & & Evoked Cel & otentials & & & & & & & & & & & & & \\
\hline 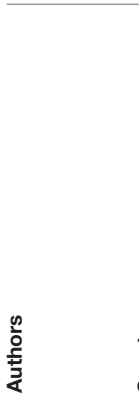 & $\begin{array}{l}\frac{\infty}{0} \\
\frac{\Phi}{0} \\
\dot{\infty}\end{array}$ & 竞 & 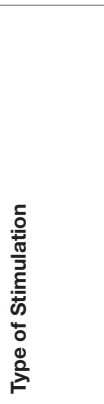 & 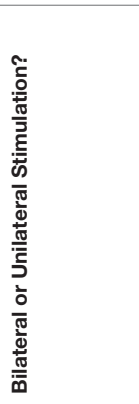 & 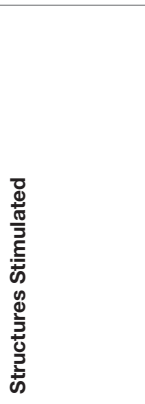 & 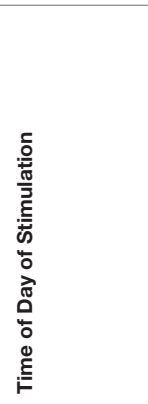 & 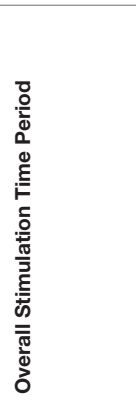 & 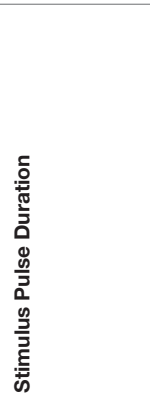 & 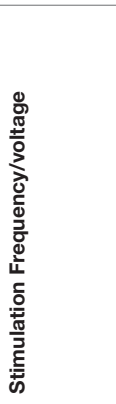 & 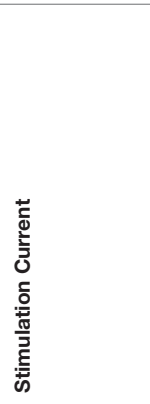 & 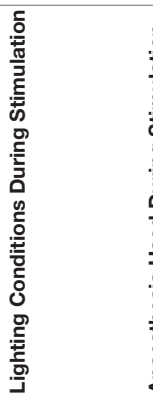 & 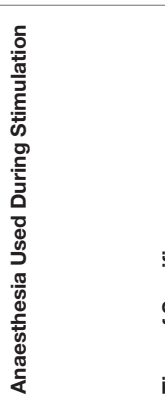 & 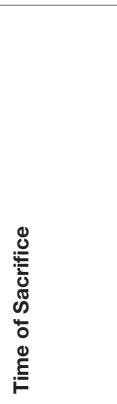 & 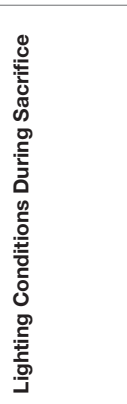 & 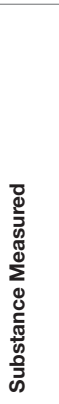 & 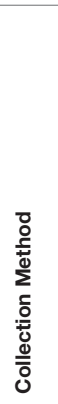 \\
\hline $\begin{array}{l}\text { Brooks et al., } \\
1975\end{array}$ & Rat & White & $\begin{array}{l}\text { Invasive } \\
\text { electrical }\end{array}$ & Bilateral & $\begin{array}{l}\text { Cervical } \\
\text { sympathetic } \\
\text { trunks }\end{array}$ & $\begin{array}{l}\text { Not } \\
\text { described }\end{array}$ & $48 \mathrm{~s}$ & $\begin{array}{l}1 \text { every } \\
1.25 \mathrm{~s}\end{array}$ & $30 \mathrm{~Hz}$ & $\begin{array}{l}\text { Not } \\
\text { described }\end{array}$ & $\begin{array}{l}\text { Not } \\
\text { described }\end{array}$ & Urethane & $\begin{array}{l}\text { Not } \\
\text { described }\end{array}$ & $\begin{array}{l}\text { Not } \\
\text { described }\end{array}$ & N/A & N/A \\
\hline $\begin{array}{l}\text { Ronnekleiv } \\
\text { et al., } 1980\end{array}$ & Rat & $\begin{array}{l}\text { Sprague } \\
\text { Dawley }\end{array}$ & $\begin{array}{l}\text { Invasive } \\
\text { Electrical }\end{array}$ & $\begin{array}{l}\text { Not } \\
\text { described }\end{array}$ & $\begin{array}{l}\text { Medial } \\
\text { habenular } \\
\text { nucleus }\end{array}$ & $\begin{array}{l}\text { Usually > } \\
\text { 18:00 }\end{array}$ & $\begin{array}{l}\text { Not } \\
\text { described }\end{array}$ & $\begin{array}{l}50- \\
200 \mu \mathrm{s}\end{array}$ & $\begin{array}{l}\text { Up to } \\
10 \mathrm{~Hz}\end{array}$ & $\begin{array}{l}0.001- \\
0.1 \mathrm{~mA}\end{array}$ & $\begin{array}{l}\text { Darkened } \\
\text { room }\end{array}$ & Urethane & $\begin{array}{l}\text { Following } \\
\text { stimulation }\end{array}$ & $\begin{array}{l}\text { Not } \\
\text { described }\end{array}$ & N/A & N/A \\
\hline Pazo, 1981 & Rat & Wistar & $\begin{array}{l}\text { Invasive } \\
\text { electrical }\end{array}$ & $\begin{array}{l}\text { Habenular } \\
\text { complex: } \\
\text { bilaterally, } \\
\text { sciatic nerve: } \\
\text { unilaterally, } \\
\text { septal area: } \\
\text { unclear, optic } \\
\text { tract: unclear }\end{array}$ & $\begin{array}{l}\text { Sciatic nerve, } \\
\text { habenular } \\
\text { complex and } \\
\text { adjacent stria } \\
\text { medullary, } \\
\text { septal area, } \\
\text { optic tract }\end{array}$ & $\begin{array}{l}10: 00- \\
18: 00\end{array}$ & $\begin{array}{l}\text { Not } \\
\text { described }\end{array}$ & $0.5 \mathrm{~ms}$ & $\begin{array}{l}\text { Not } \\
\text { described }\end{array}$ & $\begin{array}{l}\text { Variable } \\
\text { intensity }\end{array}$ & $\begin{array}{l}\text { Relatively dark } \\
\text { room with no } \\
\text { direct light on } \\
\text { the animals }\end{array}$ & Ether & $\begin{array}{l}\text { Not } \\
\text { described }\end{array}$ & $\begin{array}{l}\text { Not } \\
\text { described }\end{array}$ & N/A & N/A \\
\hline $\begin{array}{l}\text { Semm et al., } \\
1981\end{array}$ & $\begin{array}{l}\text { Guinea- } \\
\text { pig }\end{array}$ & N/A & $\begin{array}{l}\text { Invasive } \\
\text { electrical }\end{array}$ & Bilateral & $\begin{array}{l}\text { Lateral } \\
\text { habenular } \\
\text { nuclei }\end{array}$ & Daytime & $\begin{array}{l}50-100 \mathrm{~ms} \\
\text { (train) }\end{array}$ & $0.5 \mathrm{~ms}$ & $100 \mathrm{~Hz}$ & $0.5 \mathrm{~mA}$ & $\begin{array}{l}\text { Darkened } \\
\text { room }\end{array}$ & $\begin{array}{l}\text { Urethane, } \\
\text { pentobarbitone. } \\
\text { glucose, } \\
\text { gallamine } \\
\text { triethiodide } \\
\text { mixture }\end{array}$ & $\begin{array}{l}\text { Not } \\
\text { described }\end{array}$ & $\begin{array}{l}\text { Not } \\
\text { described }\end{array}$ & N/A & N/A \\
\hline $\begin{array}{l}\text { Bowers and } \\
\text { Zigmond, } \\
1982\end{array}$ & Rat & $\begin{array}{l}\text { Sprague } \\
\text { Dawley }\end{array}$ & $\begin{array}{l}\text { Invasive } \\
\text { Electrical }\end{array}$ & Bilateral & $\begin{array}{l}\text { Cervical } \\
\text { sympathetic } \\
\text { trunks }\end{array}$ & Night-time & $\begin{array}{l}\text { Not } \\
\text { described }\end{array}$ & $\begin{array}{l}0.5,1.0,3.0, \\
5.0, \text { and } 20 \mathrm{~ms}\end{array}$ & ${ }_{\mathrm{s}}^{10 \mathrm{~Hz}}$ & $\sim 5-60 \mu \mathrm{A}$ & $\begin{array}{l}\text { Not } \\
\text { described }\end{array}$ & $\begin{array}{l}\text { Chloral } \\
\text { Hydrate }\end{array}$ & $\begin{array}{l}\text { Not } \\
\text { described }\end{array}$ & $\begin{array}{l}\text { Not } \\
\text { described }\end{array}$ & N/A & N/A \\
\hline $\begin{array}{l}\text { Reuss et al., } \\
1984\end{array}$ & Rat & $\begin{array}{l}\text { Not } \\
\text { described }\end{array}$ & $\begin{array}{l}\text { Invasive } \\
\text { Electrical }\end{array}$ & Bilateral & $\begin{array}{l}\text { Lateral } \\
\text { habenular } \\
\text { nuclei }\end{array}$ & Daytime & $\begin{array}{l}\text { Not } \\
\text { described }\end{array}$ & $\begin{array}{l}0.1- \\
0.5 \mathrm{~ms}\end{array}$ & $1-10 \mathrm{~Hz}$ & $\begin{array}{l}0.1-0.5 \mathrm{~mA} \\
\text { (occasionally } \\
\text { up to } 5 \mathrm{~mA} \text { ) }\end{array}$ & $\begin{array}{l}\text { Darkened } \\
\text { room }\end{array}$ & $\begin{array}{l}\text { Urethane and } \\
\text { pentobarbital }\end{array}$ & $\begin{array}{l}\text { Not } \\
\text { described }\end{array}$ & $\begin{array}{l}\text { Not } \\
\text { described }\end{array}$ & N/A & N/A \\
\hline $\begin{array}{l}\text { Reuss et al., } \\
1985 \mathrm{~b}\end{array}$ & Rat & $\begin{array}{l}\text { Sprague } \\
\text { Dawley }\end{array}$ & $\begin{array}{l}\text { Invasive } \\
\text { electrical }\end{array}$ & Both & SCG & 09:00-18:00 & $\begin{array}{l}\text { Not } \\
\text { described }\end{array}$ & $0.2 \mathrm{~ms}$ & $10 \mathrm{~Hz}$ & $0.1-0.5 \mathrm{~mA}$ & $\begin{array}{l}\text { "Natural } \\
\text { lighting } \\
\text { conditions" }\end{array}$ & Urethane & $\begin{array}{l}\text { Not } \\
\text { described }\end{array}$ & $\begin{array}{l}\text { Not } \\
\text { described }\end{array}$ & N/A & N/A \\
\hline $\begin{array}{l}\text { Reyes- } \\
\text { Vazquez et al., } \\
1986\end{array}$ & Rat & $\begin{array}{l}\text { Sprague } \\
\text { Dawley }\end{array}$ & $\begin{array}{l}\text { Invasive } \\
\text { electrical }\end{array}$ & Bilateral & $\begin{array}{l}\text { Postganglionic } \\
\text { nerve fibres of } \\
\text { the SCG }\end{array}$ & $\begin{array}{l}\text { Not } \\
\text { described }\end{array}$ & $\begin{array}{l}\text { Not } \\
\text { described }\end{array}$ & $0.2 \mathrm{~ms}$ & $1 \mathrm{~Hz}$ & $\begin{array}{l}0.1- \\
0.6 \mathrm{~mA}\end{array}$ & $\begin{array}{l}\text { Ordinary room } \\
\text { illumination } \\
\text { during light } \\
\text { cycle }\end{array}$ & Urethane & $\begin{array}{l}\text { Following } \\
\text { stimulation }\end{array}$ & $\begin{array}{l}\text { Not } \\
\text { described }\end{array}$ & N/A & $\mathrm{N} / \mathrm{A}$ \\
\hline $\begin{array}{l}\text { Stehle et al., } \\
1987\end{array}$ & Hamster & r Golden & $\begin{array}{l}\text { Invasive } \\
\text { electrical }\end{array}$ & $\begin{array}{l}\text { SCG: } \\
\text { unilateral, } \\
\text { habenular } \\
\text { nuclei: } \\
\text { bilateral }\end{array}$ & $\begin{array}{l}\text { SCG and } \\
\text { lateral } \\
\text { habenular } \\
\text { nuclei }\end{array}$ & $\begin{array}{l}\text { During the } \\
\text { dark and light } \\
\text { periods }\end{array}$ & $<17 \mathrm{~h}$ & $0.2 \mathrm{~ms}$ & $2-20 \mathrm{~Hz}$ & $\begin{array}{l}0.2 \mathrm{~mA} \text { for } \\
\text { habenular and } \\
\text { optic but up to } \\
2 \mathrm{~mA} \text { for the } \\
\text { SCG }\end{array}$ & $\begin{array}{l}\text { Not } \\
\text { described }\end{array}$ & Urethane & $\begin{array}{l}\text { Not } \\
\text { described }\end{array}$ & $\begin{array}{l}\text { Not } \\
\text { described }\end{array}$ & N/A & $\mathrm{N} / \mathrm{A}$ \\
\hline
\end{tabular}


TABLE 2 | Continued

\begin{tabular}{|c|c|c|c|c|c|c|c|c|c|c|c|c|c|c|c|c|}
\hline & Evoked & Cell Poter & ntials & & & & & & & & & & & & & \\
\hline 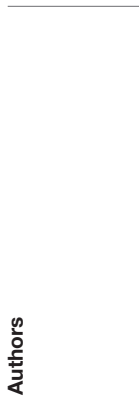 & $\begin{array}{l}\frac{\mathscr{\omega}}{0} \\
\frac{\Phi}{\infty} \\
\dot{\infty}\end{array}$ & 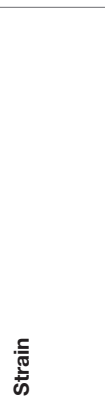 & 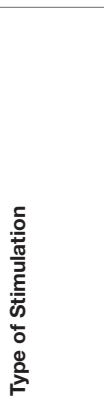 & 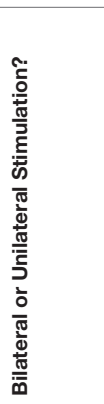 & 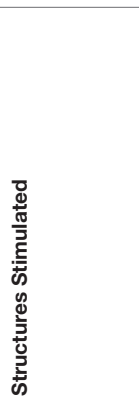 & 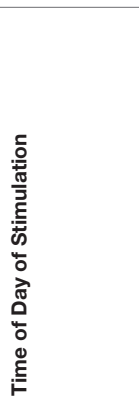 & 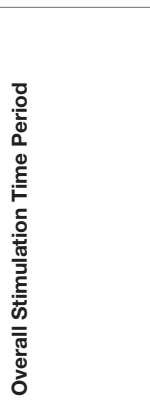 & 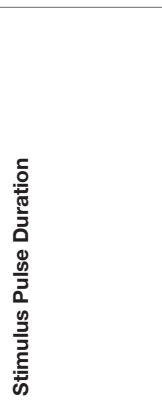 & 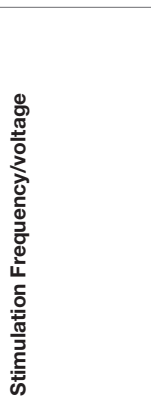 & 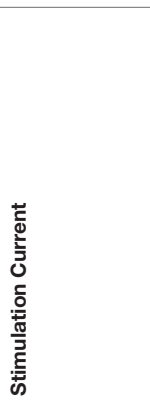 & 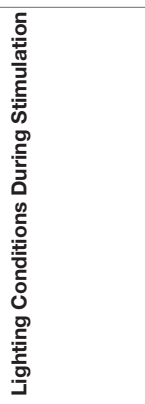 & 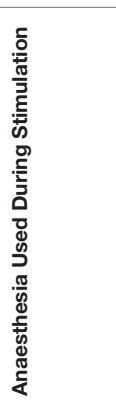 & 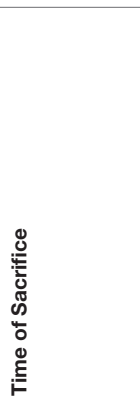 & 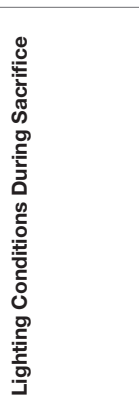 & 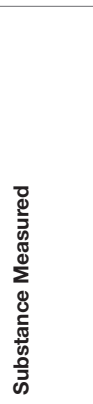 & 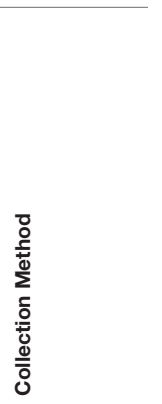 \\
\hline $\begin{array}{l}\text { Patel and } \\
\text { Demaine, } \\
1990\end{array}$ & Hamster & Golden & $\begin{array}{l}\text { Invasive } \\
\text { electrical }\end{array}$ & Both & SCG & $\begin{array}{l}\text { up to } \\
6 \mathrm{~h} \text { after } \\
15: 30\end{array}$ & Not described & $0.5-1 \mathrm{~ms}$ & $10-20 \mathrm{~Hz}$ & $0.5-1 \mathrm{~mA}$ & $\begin{array}{l}\text { Artificial lab } \\
\text { lighting }\end{array}$ & Urethane & $\begin{array}{l}\text { Not } \\
\text { described }\end{array}$ & $\begin{array}{l}\text { Not } \\
\text { described }\end{array}$ & N/A & N/A \\
\hline $\begin{array}{l}\text { Pazo and } \\
\text { Gonzalez, } \\
1991\end{array}$ & Rat & Wistar & $\begin{array}{l}\text { Invasive } \\
\text { electrical }\end{array}$ & Unilateral & $\begin{array}{l}\text { SCG and } \\
\text { sciatic nerve }\end{array}$ & 09:00-18:00 & Not described & $0.5 \mathrm{~ms}$ & Not described & Up to $0.5 \mathrm{~mA}$ & $\begin{array}{l}\text { "Relatively } \\
\text { dark room } \\
\text { with } \\
\text { no direct } \\
\text { light } \\
\text { on the } \\
\text { animal } \\
\text { during } \\
\text { daylight" }\end{array}$ & Urethane & $\begin{array}{l}\text { Not } \\
\text { described }\end{array}$ & $\begin{array}{l}\text { Not } \\
\text { described }\end{array}$ & N/A & N/A \\
\hline \multicolumn{17}{|c|}{ Indoleamine Output and Enzymatic Activity } \\
\hline $\begin{array}{l}\text { Brownstein } \\
\text { and Heller, } \\
1968\end{array}$ & Rat & Holtzman & $\begin{array}{l}\text { Invasive } \\
\text { electrical }\end{array}$ & Unilateral & $\begin{array}{l}\text { Preganglionic } \\
\text { cervical } \\
\text { sympathetic } \\
\text { fibres }\end{array}$ & $\begin{array}{l}\text { Not } \\
\text { described }\end{array}$ & $4 \mathrm{~h}$ & $9 \mathrm{~s}$ on; $51 \mathrm{~s}$ off & $10 \mathrm{~Hz}$ & $3-5 \mathrm{~mA}$ & $\begin{array}{l}\text { Not } \\
\text { described but } \\
\text { animals } \\
\text { blinded }\end{array}$ & Ether & 4-h post-op & $\begin{array}{l}\text { Not } \\
\text { described } \\
\text { but animals } \\
\text { blinded }\end{array}$ & HIOMT & $\begin{array}{l}\text { Pineal } \\
\text { homogenization }\end{array}$ \\
\hline $\begin{array}{l}\text { Volkman and } \\
\text { Heller, } 1971\end{array}$ & Rat & Holtzman & $\begin{array}{l}\text { Invasive } \\
\text { electrical }\end{array}$ & Unilateral & $\begin{array}{l}\text { Preganglionic } \\
\text { cervical } \\
\text { sympathetic } \\
\text { trunk }\end{array}$ & $\begin{array}{l}\text { Daytime/ } \\
\text { light phase }\end{array}$ & 1,2 , or $3 \mathrm{~h}$ & $\begin{array}{l}10 \mathrm{~ms} \text { for } 9 \mathrm{~s} \\
\text { every min }\end{array}$ & $10 \mathrm{~Hz}$ & $\begin{array}{l}2 x \text { that } \\
\text { required to } \\
\text { produce } \\
\text { maximal } \\
\text { exophthalmos } \\
\text { in the eye } \\
(2 \times 0.23 \mathrm{~mA})\end{array}$ & $\begin{array}{l}\text { Not } \\
\text { described }\end{array}$ & Ether & $\begin{array}{l}\text { Immediately } \\
\text { or } 1 \text {-h post- } \\
\text { stimulation } \\
\text { cessation }\end{array}$ & $\begin{array}{l}\text { Not } \\
\text { described }\end{array}$ & AANAT & $\begin{array}{l}\text { Pineal } \\
\text { homogenization }\end{array}$ \\
\hline $\begin{array}{l}\text { Bowers and } \\
\text { Zigmond, } \\
1980\end{array}$ & Rat & $\begin{array}{l}\text { Sprague } \\
\text { Dawley }\end{array}$ & $\begin{array}{l}\text { Invasive } \\
\text { electrical }\end{array}$ & Bilateral & $\begin{array}{l}\text { Cervical } \\
\text { sympathetic } \\
\text { trunks }\end{array}$ & $\begin{array}{l}>4 \mathrm{~h} \text { after } \\
\text { darkness } \\
\text { onset }\end{array}$ & $\begin{array}{l}\text { Animals were } \\
\text { not stimulated } \\
\text { past the time } \\
\text { were lights } \\
\text { would } \\
\text { normally turn } \\
\text { on }\end{array}$ & $0.5 \mathrm{~ms}$ & $5 \mathrm{~Hz}$ & $0.4-2.0 \mathrm{~mA}$ & $\begin{array}{l}\text { Exposed to } \\
\text { light for 15 } \\
\text { min prior to } \\
\text { anaesthetic } \\
\text { administration } \\
\text { for SCG } \\
\text { exposure to } \\
\text { reduce } \\
\text { night-time } \\
\text { AANAT levels } \\
\text { by more than } \\
\text { 95\% }\end{array}$ & $\begin{array}{l}\text { Chloral } \\
\text { hydrate }\end{array}$ & $\begin{array}{l}\text { Immediately } \\
\text { post- } \\
\text { stimulation - } \\
\text { before the } \\
\text { onset of the } \\
\text { light period }\end{array}$ & Dim red light & AANAT & $\begin{array}{l}\text { Pineal } \\
\text { homogenization }\end{array}$ \\
\hline $\begin{array}{l}\text { Heydorn } \\
\text { et al., } 1981\end{array}$ & $\begin{array}{l}\text { Rat } \\
\text { (ex vivo) }\end{array}$ & $\begin{array}{l}\text { Sprague } \\
\text { Dawley }\end{array}$ & $\begin{array}{l}\text { Invasive } \\
\text { electrical }\end{array}$ & Bilateral & SCG & $\begin{array}{l}\text { Not } \\
\text { described }\end{array}$ & $1 \mathrm{~min}$ & $10 \mathrm{~ms}$ & $10 \mathrm{~Hz}(10 \mathrm{~V})$ & $\begin{array}{l}\text { Not } \\
\text { described }\end{array}$ & $\begin{array}{l}\text { Not } \\
\text { described }\end{array}$ & $\begin{array}{l}\text { Not } \\
\text { described }\end{array}$ & $\begin{array}{l}\text { Not } \\
\text { described }\end{array}$ & $\begin{array}{l}\text { Not } \\
\text { described }\end{array}$ & CAMP & $\begin{array}{l}\text { Pineal } \\
\text { homogenization }\end{array}$ \\
\hline
\end{tabular}


TABLE 2 | Continued

\begin{tabular}{|c|c|c|c|c|c|c|c|c|c|c|c|c|c|c|c|c|}
\hline & & oked Cell & otentials & & & & & & & & & & & & & \\
\hline 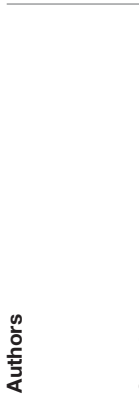 & $\begin{array}{l}\frac{\mathscr{0}}{0} \\
\frac{0}{0} \\
\text { के }\end{array}$ & $\begin{array}{l}\text { 琣 } \\
\text { के }\end{array}$ & 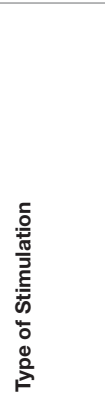 & 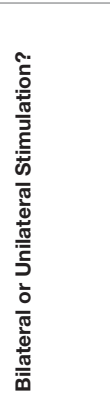 & 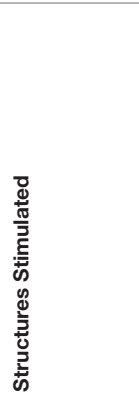 & 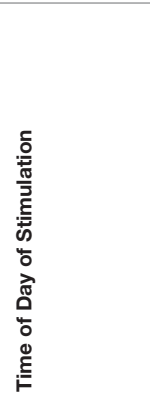 & 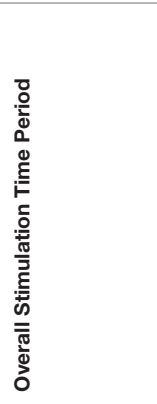 & 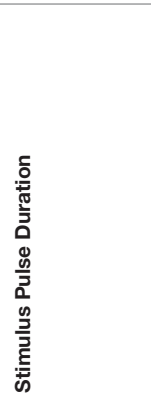 & 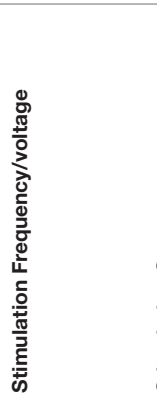 & 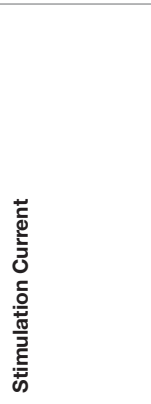 & 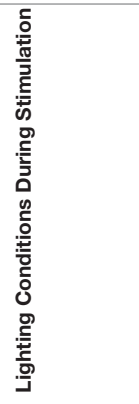 & 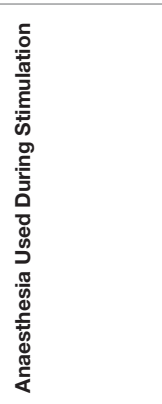 & 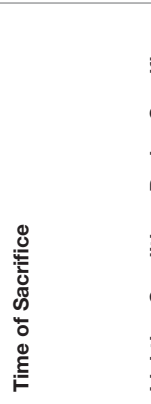 & 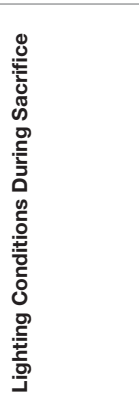 & 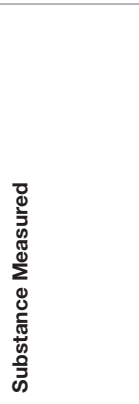 & 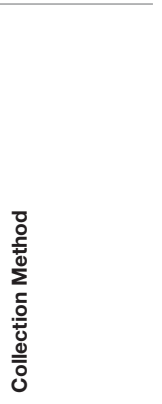 \\
\hline $\begin{array}{l}\text { Bowers and } \\
\text { Zigmond, } \\
1982\end{array}$ & Rat & $\begin{array}{l}\text { Sprague } \\
\text { Dawley }\end{array}$ & $\begin{array}{l}\text { Invasive } \\
\text { electrical }\end{array}$ & Both & $\begin{array}{l}\text { Cervical } \\
\text { sympathetic } \\
\text { trunks }\end{array}$ & $\begin{array}{l}\text { Night }(>4 \mathrm{~h} \\
\text { into } \\
\text { night period) } \\
\text { AND day } \\
\text { ( }>4 \mathrm{~h} \\
\text { into } \\
\text { light } \\
\text { period) }\end{array}$ & $\begin{array}{l}0.5,1,1.5 \text {, and } \\
2 \mathrm{~h}\end{array}$ & $\mathrm{~d} 0.5 \mathrm{~ms}$ & $10 \mathrm{~Hz}$ & $\begin{array}{l}2 x \text { that } \\
\text { require to } \\
\text { produce } \\
\text { maximal } \\
\text { exophthalmos } \\
\text { of the ipsilateral } \\
\text { eye (values } \\
\text { ranged for each } \\
\text { nerve from } \\
100-10004 \text { ) }\end{array}$ & $\begin{array}{l}\text { Not } \\
\text { described }\end{array}$ & $\begin{array}{l}\text { Chloral } \\
\text { hydrate }\end{array}$ & $\begin{array}{l}\text { Immediately } \\
\text { following } \\
\text { stimulation }\end{array}$ & $\begin{array}{l}\text { Not } \\
\text { described }\end{array}$ & AANAT & $\begin{array}{l}\text { Pineal } \\
\text { homogenization }\end{array}$ \\
\hline $\begin{array}{l}\text { Bowers et al., } \\
1984 a\end{array}$ & & $\begin{array}{l}\text { Sprague } \\
\text { Dawley }\end{array}$ & $\begin{array}{l}\text { Invasive } \\
\text { electrical }\end{array}$ & Bilateral & $\begin{array}{l}\text { Cervical } \\
\text { sympathetic } \\
\text { trunks }\end{array}$ & $\begin{array}{l}>4 \mathrm{hinto} \\
\text { light period }\end{array}$ & $3 \mathrm{~h}$ & $\begin{array}{l}\text { Not } \\
\text { described }\end{array}$ & $5 \mathrm{~Hz}$ & $\begin{array}{l}2 x \text { that } \\
\text { required to } \\
\text { produce } \\
\text { maximal } \\
\text { exophthalmos } \\
\text { in the ipsilateral } \\
\text { eye }\end{array}$ & $\begin{array}{l}>4 \mathrm{~h} \text { into } \\
\text { light } \\
\text { period } \\
\text { al }\end{array}$ & $\begin{array}{l}\text { Chloral } \\
\text { hydrate }\end{array}$ & $\begin{array}{l}>7 \mathrm{~h} \text { into light } \\
\text { period }\end{array}$ & Dimred light & AANAT & $\begin{array}{l}\text { Pineal } \\
\text { homogenization }\end{array}$ \\
\hline $\begin{array}{l}\text { Reuss et al, } \\
1985 a\end{array}$ & Rats & $\begin{array}{l}\text { Sprague } \\
\text { Dawley }\end{array}$ & $\begin{array}{l}\text { Invasive } \\
\text { electrical }\end{array}$ & Bilateral & PVN & $\begin{array}{l}\text { Daytime } \\
\text { experiments: } \\
\text { 11:00-13:00; } \\
\text { Night-time } \\
\text { experiments: } \\
\text { 00:00-06:00 }\end{array}$ & $15,30,60$ min & $0.2 \mathrm{~ms}$ & $10 \mathrm{~Hz}$ & $0.1 \mathrm{~mA}$ & $\begin{array}{l}\text { Normal } \\
\text { artificial light }\end{array}$ & Urethane & $\begin{array}{l}\text { Following } \\
\text { stimulation }\end{array}$ & $\begin{array}{l}\text { Normal } \\
\text { artificial light } \\
\text { but animals } \\
\text { blinded for } \\
\text { night-time } \\
\text { experiments }\end{array}$ & $\begin{array}{l}\text { AANAT, } \\
\text { melatonin }\end{array}$ & $\begin{array}{l}\text { Pineal } \\
\text { homogenization }\end{array}$ \\
\hline $\begin{array}{l}\text { Olcese et al., } \\
1987\end{array}$ & Rat & $\begin{array}{l}\text { Sprague } \\
\text { Dawley }\end{array}$ & $\begin{array}{l}\text { Invasive } \\
\text { electrical }\end{array}$ & Bilateral & PVN & $>02: 00$ & $2 \mathrm{~min}$ & $0.2 \mathrm{~ms}$ & $10 \mathrm{~Hz}$ & $0.1 \mathrm{~mA}$ & $\begin{array}{l}\text { Yes, but all } \\
\text { animals } \\
\text { blinded } \\
\text { surgically }\end{array}$ & Urethane & $\begin{array}{l}30 \text { min post- } \\
\text { stimulation }\end{array}$ & $\begin{array}{l}\text { Artificial light } \\
\text { but animals } \\
\text { are blinded }\end{array}$ & Melatonin, NE & $\begin{array}{l}\text { Pineal } \\
\text { homogenization }\end{array}$ \\
\hline $\begin{array}{l}\text { Reuss et al., } \\
1989\end{array}$ & Rat & $\begin{array}{l}\text { Sprague } \\
\text { Dawley }\end{array}$ & $\begin{array}{l}\text { Invasive } \\
\text { electrical }\end{array}$ & Bilateral & SCG & 10:00-14:00 & $\begin{array}{l}\text { (1) } 120 \mathrm{~min} ; \\
\text { (2a + b) } 15 \mathrm{~min}\end{array}$ & $\begin{array}{l}\text { (1) } 0.5 \mathrm{~ms} \text {; (2a) } \\
\text { n } 0.5 \mathrm{~ms} \text {; (2b) } \\
1 \mathrm{~ms}\end{array}$ & $\begin{array}{l}\text { (1) } 10 \mathrm{~Hz} \text {; (2a) } \\
10 \mathrm{~Hz} \text {; (2b) } \\
25 \mathrm{~Hz}\end{array}$ & $\begin{array}{l}\text { (1) } 0.5 \mathrm{~mA} \text {; } \\
(2 \mathrm{a}+\mathrm{b}) \\
0.5 \mathrm{~mA}\end{array}$ & $\begin{array}{l}\text { Not } \\
\text { described }\end{array}$ & Urethane & $\begin{array}{l}\text { (1) } 2 \mathrm{~h} \text { after } \\
\text { stimulation } \\
\text { onset; (2) } \\
\text { immediately } \\
\text { following } \\
\text { stimulation }\end{array}$ & $\begin{array}{l}\text { Not } \\
\text { described }\end{array}$ & AANAT & $\begin{array}{l}\text { Pineal } \\
\text { homogenization } \\
\text { of } 2 / 3 \text { of each } \\
\text { gland }\end{array}$ \\
\hline $\begin{array}{l}\text { Chan et al., } \\
1989\end{array}$ & Rabbit & $\begin{array}{l}\text { New } \\
\text { Zealand }\end{array}$ & $\begin{array}{l}\text { Invasive } \\
\text { electrical }\end{array}$ & Unilateral & $\begin{array}{l}\text { Left } \\
\text { preganglionic } \\
\text { cervical } \\
\text { sympathetic } \\
\text { trunk }\end{array}$ & $\begin{array}{l}\text { Light } \\
\text { phase }\end{array}$ & $\begin{array}{l}24- \\
60 \mathrm{~min}\end{array}$ & $\begin{array}{l}60 \mathrm{~ms} \text { every } \\
2 \mathrm{~s} \text { OR } 7.5 \mathrm{~s} \\
\text { every } 20 \mathrm{~s}\end{array}$ & $300 \mathrm{~Hz}$ & $1-5 \mathrm{~mA}$ & $\begin{array}{l}\text { Not } \\
\text { described }\end{array}$ & Pentobarbital & $\begin{array}{l}\text { Not } \\
\text { described }\end{array}$ & $\begin{array}{l}\text { Not } \\
\text { described }\end{array}$ & Melatonin & $\begin{array}{l}\text { Blood (plasma) } \\
\text { sampling from } \\
\text { the confluens } \\
\text { sinuum }\end{array}$ \\
\hline $\begin{array}{l}\text { Lingappa and } \\
\text { Zigmond, } \\
2013\end{array}$ & & $\begin{array}{l}\text { Sprague } \\
\text { Dawley }\end{array}$ & $\begin{array}{l}\text { Invasive } \\
\text { electrical }\end{array}$ & Bilateral & $\begin{array}{l}\text { Cervical } \\
\text { sympathetic } \\
\text { trunks }\end{array}$ & $\begin{array}{l}4-8 \mathrm{~h} \text { into } \\
\text { daytime }\end{array}$ & $3 \mathrm{~h}$ & $0.5 \mathrm{~ms}$ & $\begin{array}{l}10,5,2.5, \text { and } \\
1 \mathrm{~Hz}(10 \mathrm{~Hz} \\
\text { was considered } \\
\text { optimal for } \\
\text { AANAT } \\
\text { stimulation) }\end{array}$ & d & $\begin{array}{l}\text { Not } \\
\text { described }\end{array}$ & Chloral hydrate & $\begin{array}{l}\text { Immediately } \\
\text { after } \\
\text { stimulation } \\
\text { (stimulation } \\
\text { carried out } \\
4-8 \mathrm{~h} \text { into } \\
\text { daytime) }\end{array}$ & $\begin{array}{l}\text { Not } \\
\text { described }\end{array}$ & AANAT & $\begin{array}{l}\text { Pineal } \\
\text { homogenization }\end{array}$ \\
\hline
\end{tabular}




\begin{tabular}{|c|c|c|c|c|c|c|c|c|c|c|c|c|c|c|c|c|}
\hline $\begin{array}{l}\frac{0}{0} \\
\frac{1}{5} \\
\frac{1}{4}\end{array}$ & 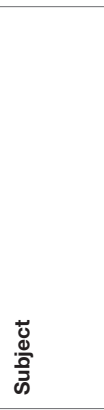 & $\begin{array}{l}\frac{\mathscr{\omega}}{\overline{0}} \\
\frac{\delta}{\omega}\end{array}$ & 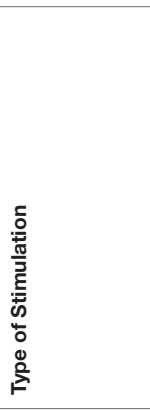 & 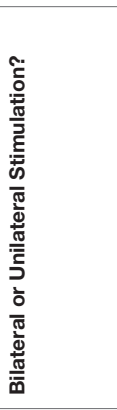 & 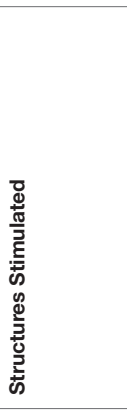 & 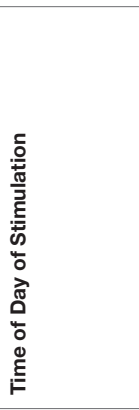 & 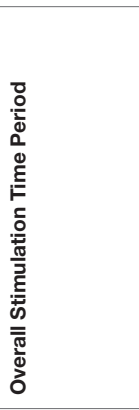 & 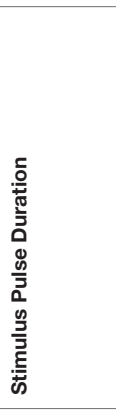 & 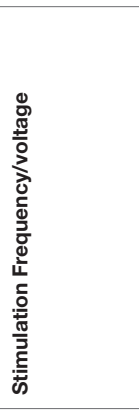 & 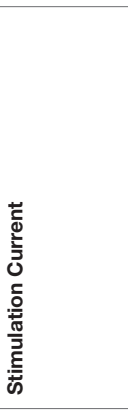 & 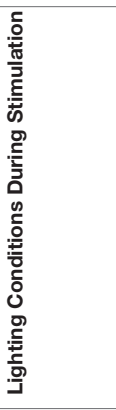 & 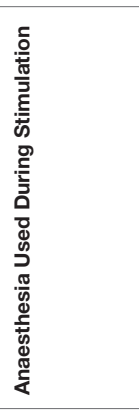 & 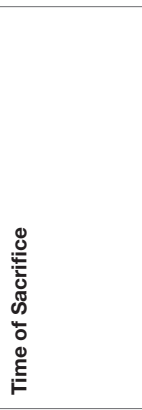 & 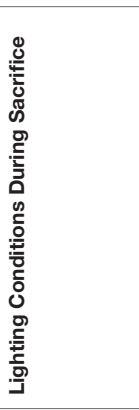 & 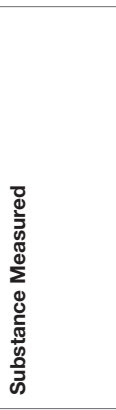 & 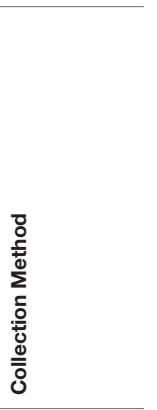 \\
\hline \multicolumn{17}{|c|}{ Non-invasive Stimulation } \\
\hline $\begin{array}{l}\text { Mclntyre and } \\
\text { Oxenkrug, } \\
1984\end{array}$ & Rat & $\begin{array}{l}\text { Sprague } \\
\text { Dawley }\end{array}$ & $\begin{array}{l}\text { Non-invasive } \\
\text { electrical }\end{array}$ & Bilateral & Ears & Before 12:00 & $\begin{array}{l}\text { One shock } \\
\text { every day for } \\
7 \text { days }\end{array}$ & $0.75 \mathrm{~s}$ & $130 \mathrm{~V}$ & $\begin{array}{l}\text { Not } \\
\text { described }\end{array}$ & $\begin{array}{l}\text { Not } \\
\text { described }\end{array}$ & $\begin{array}{l}\text { Not } \\
\text { described }\end{array}$ & $2100 \mathrm{~h}$ & Dim red light & $\begin{array}{l}\text { 5-HT, } \\
\text { melatonin, } \\
\text { NAS }\end{array}$ & $\begin{array}{l}\text { Pineal } \\
\text { homogenization }\end{array}$ \\
\hline $\begin{array}{l}\text { Nowak et al., } \\
1988\end{array}$ & Rat & Wistar & $\begin{array}{l}\text { Non-invasive } \\
\text { electrical }\end{array}$ & Bilateral & Ears & $\begin{array}{l}\text { At the end } \\
\text { of the light } \\
\text { phase with } \\
\text { the very } \\
\text { final } \\
\text { treatment } \\
\text { being given } \\
\text { at 22:00 }\end{array}$ & $\begin{array}{l}\text { A single } \\
\text { electric } \\
\text { sock or } 10 \\
\text { shocks per } \\
\text { day over } 10 \\
\text { consecutive } \\
\text { days }\end{array}$ & $500 \mathrm{~ms}$ & $50 \mathrm{~Hz}$ & $\begin{array}{l}70 \mathrm{~mA} \text { (to } \\
\text { induce } \\
\text { tonic-clonic } \\
\text { seizures) }\end{array}$ & $\begin{array}{l}\text { Not } \\
\text { described }\end{array}$ & $\begin{array}{l}\text { Not } \\
\text { described }\end{array}$ & $\begin{array}{l}1 \text { or } 2 \mathrm{~h} \text { after } \\
\text { the final } \\
\text { stimulation }\end{array}$ & $\begin{array}{l}\text { Light or } \\
\text { dim-red light }\end{array}$ & $\begin{array}{l}\text { AANAT, } \\
\text { HIOMT }\end{array}$ & $\begin{array}{l}\text { Pineal } \\
\text { homogenization }\end{array}$ \\
\hline $\begin{array}{l}\text { Oxenkrug } \\
\text { et al., } 1991\end{array}$ & Rat & $\begin{array}{l}\text { Sprague } \\
\text { Dawley }\end{array}$ & $\begin{array}{l}\text { Non-invasive } \\
\text { electrical }\end{array}$ & Bilateral & Ears & 10:00 & $\begin{array}{l}\text { Not } \\
\text { described }\end{array}$ & $0.75 \mathrm{~s}$ & $130 \mathrm{~V}$ & $\begin{array}{l}\text { Not } \\
\text { described }\end{array}$ & $\begin{array}{l}\text { Not } \\
\text { described }\end{array}$ & $\begin{array}{l}\text { Not } \\
\text { described }\end{array}$ & $\begin{array}{l}90 \text { min after } \\
\text { stimulation }\end{array}$ & $\begin{array}{l}\text { Not } \\
\text { described }\end{array}$ & $\begin{array}{l}\text { Melatonin, } \\
\text { serotonin, } \\
5-\text { HIAA. } \\
\text { NAS }\end{array}$ & $\begin{array}{l}\text { Pineal } \\
\text { homogenization }\end{array}$ \\
\hline $\begin{array}{l}\text { Chao et al., } \\
2001\end{array}$ & Rat & Wistar & $\begin{array}{l}\text { Percutaneous } \\
\text { electrical } \\
\text { nerve } \\
\text { stimulation }\end{array}$ & $\mathrm{N} / \mathrm{A}$ & $\begin{array}{l}\text { Fengfu } \\
\text { DU16 and } \\
\text { Jinsuo DU8 }\end{array}$ & $\begin{array}{l}\text { Not } \\
\text { described }\end{array}$ & 30 or $60 \mathrm{~min}$ & $\begin{array}{l}\text { Not } \\
\text { described }\end{array}$ & $80 \mathrm{~Hz}$ & $1.7-2.5 \mathrm{~mA}$ & $\begin{array}{l}\text { Not } \\
\text { described }\end{array}$ & $\begin{array}{l}\text { Sodium } \\
\text { pentobarbital }\end{array}$ & $\begin{array}{l}\text { After } \\
\text { stimulation }\end{array}$ & $\begin{array}{l}\text { Not } \\
\text { described }\end{array}$ & Melatonin & $\begin{array}{l}\text { Pineal } \\
\text { homogenization }\end{array}$ \\
\hline $\begin{array}{l}\text { Spence et al., } \\
2004\end{array}$ & Humans & $\begin{array}{l}\text { Humans } \\
\text { with anxiety } \\
\text { and } \\
\text { insomnia } \\
\text { (but no } \\
\text { diagnosed } \\
\text { anxiety } \\
\text { disorder) }\end{array}$ & Acupuncture & $\begin{array}{l}\text { Not } \\
\text { described }\end{array}$ & $\begin{array}{l}\text { Not } \\
\text { described }\end{array}$ & $\begin{array}{l}\text { Not } \\
\text { described }\end{array}$ & $\begin{array}{l}2 \times \text { a week } \\
\text { for } 5 \text { weeks }\end{array}$ & $\begin{array}{l}1 \mathrm{hr} \text { per } \\
\text { session }\end{array}$ & N/A & $\mathrm{N} / \mathrm{A}$ & $N / A$ & N/A & N/A & $\mathrm{N} / \mathrm{A}$ & Melatonin & $\begin{array}{l}\text { Measurement } \\
\text { of urinary } \\
\text { melatonin } \\
\text { metabolite } \\
\text { aMT6s }\end{array}$ \\
\hline $\begin{array}{l}\text { Kayumov } \\
\text { et al., } 2003\end{array}$ & Humans & $\begin{array}{l}\text { Humans } \\
\text { with } \\
\text { insomnia } \\
\text { and anxiety }\end{array}$ & Acupuncture & $\begin{array}{l}\text { Not } \\
\text { described }\end{array}$ & $\begin{array}{l}\text { Not } \\
\text { described }\end{array}$ & $\begin{array}{l}\text { Not } \\
\text { described }\end{array}$ & $\begin{array}{l}2 \times \text { a week } \\
\text { for } 5 \text { weeks }\end{array}$ & N/A & N/A & N/A & $\begin{array}{l}\text { Not } \\
\text { described }\end{array}$ & N/A & N/A & N/A & Melatonin & $\begin{array}{l}\text { Measurement } \\
\text { of urinary } \\
\text { melatonin } \\
\text { metabolite } \\
\text { aMT6s }\end{array}$ \\
\hline Li et al., 2014 & Rat & $\begin{array}{l}\text { Zucker } \\
\text { diabetic fatty } \\
\text { and Zucker } \\
\text { lean }\end{array}$ & $\begin{array}{l}\text { Non-invasive } \\
\text { electrical }\end{array}$ & Bilateral & $\begin{array}{l}\text { Right side } \\
\text { auricular } \\
\text { concha } \\
\text { region }\end{array}$ & 14:00-17:00 & $\begin{array}{l}30 \text { min for } \\
34 \\
\text { consecutive } \\
\text { days }\end{array}$ & $\begin{array}{l}\text { Not } \\
\text { described }\end{array}$ & $\begin{array}{l}2 \text { and } 15 \mathrm{~Hz} \\
\text { alternating } \\
\text { every sec }\end{array}$ & $2 \mathrm{~mA}$ & $\begin{array}{l}\text { Not } \\
\text { described }\end{array}$ & Isoflurane & $\begin{array}{l}\text { Not } \\
\text { described }\end{array}$ & $\begin{array}{l}\text { Not } \\
\text { described }\end{array}$ & Melatonin & $\begin{array}{l}\text { Blood } \\
\text { (plasma) } \\
\text { sampling from } \\
\text { the tail vein }\end{array}$ \\
\hline $\begin{array}{l}\text { Wang et al., } \\
2015\end{array}$ & Rat & $\begin{array}{l}\text { Zucker } \\
\text { diabetic fatty }\end{array}$ & $\begin{array}{l}\text { Non-invasive } \\
\text { electrical }\end{array}$ & Bilateral & $\begin{array}{l}\text { Right side } \\
\text { auricular } \\
\text { concha } \\
\text { region }\end{array}$ & Afternoon & $30 \mathrm{~min}$ & $\begin{array}{l}\text { Not } \\
\text { described }\end{array}$ & $\begin{array}{l}2 \text { and } 15 \mathrm{~Hz} \\
\text { switched } \\
\text { every sec }\end{array}$ & $2 \mathrm{~mA}$ & $\begin{array}{l}\text { Not } \\
\text { described }\end{array}$ & Isoflurane & $\begin{array}{l}\text { Not } \\
\text { described }\end{array}$ & $\begin{array}{l}\text { Not } \\
\text { described }\end{array}$ & Melatonin & $\begin{array}{l}\text { Blood } \\
\text { (plasma) } \\
\text { sampling from } \\
\text { the tail vein }\end{array}$ \\
\hline
\end{tabular}


frequency stimulation of various central structures, viscera, and vasculature can exert opposite effects (Ngai et al., 1999; StenerVictorin et al., 2006; Cakmak et al., 2008; Zhao, 2008; Liu et al., 2012; Su et al., 2018). Therefore, it is likely a frequency dependent effect exists in the stimulation of the pineal gland.

Patel and Demaine (1990) report little difference in response rates following either bilateral and unilateral SCG stimulation $(62 \%)$ in the hamster, yet a much lower response rate $(28 \%)$ is alternatively reported in the same species using unilateral stimulation (Stehle et al., 1987). The differences in response rate following unilateral stimulation may be due to the latter authors ligating and separating the confluens sinuum, which exists in close proximity to the path of the nervi conarii that innervates the pineal gland (Kappers, 1960b). Such a practice may have inadvertently disrupted sympathetic innervation to the gland, resulting in a lower response rate than would otherwise be observed with the confluens sinuum intact. The current used to stimulate the SCG could also play a role in the response rate of pinealocytes, with higher currents resulting in hyperpolarisation of postganglionic cell membranes, and therefore, a lessened response from pinealocytes. This could explain why Stehle et al. (1987) report a lower response following bilateral stimulation of the SCG in hamsters compared to Patel and Demaine (1990), as the former used a slightly higher current.

Using unilateral SCG stimulation, the nature of augmentation in pineal cell firing was investigated in rats in the daytime (Pazo and Gonzalez, 1991). In this study, half (56\%) of the responsive pinealocytes showed an inhibitory response, one third of the cells (33\%) exhibited an excitatory response, and a biphasic response was observed in $11 \%$ of cells. In addition, two other studies note similar prevalence of inhibitory and excitatory responses (61.5\% inhibitory and $46.2 \%$ excitatory $^{1}$, Stehle et al., 1987 vs. $42.9 \%$ excitatory and 57.1\% inhibitory, Reuss et al., 1985b) following unilateral stimulation of the SCG in the hamster and rat, respectively. Patel and Demaine (1990) reported an increase in cell firing rate as the most prevalent response $(47 \%)$ followed by a decrease in firing rate $(37 \%)$, and a biphasic response in $16 \%$ of cells following SCG stimulation in the hamster. They also comment on the rapidness of the electrophysiological response to electrical stimulation of the SCG, commencing within $15-50 \mathrm{~ms}$. In contrast, the pineal response to the onset of darkness is much slower, with increases in AANAT and melatonin not being apparent for several hours (Tamarkin et al., 1979, 1980) indicating that there exists a delay between the sympathetic pineal cell response and the intracellular melatonin upregulation. Similar response types and incidences were also reported following unilateral stimulation of the SCN using the same parameters (Pazo and Gonzalez, 1991). This implies that the pineal cellular responses are similar following stimulation of either the SCG or SCN. Contrary to this, unilateral stimulation of the sciatic nerve (Pazo and Gonzalez, 1991) resulted in mainly an inhibitory response, whilst stimulation of the lateral habenular nuclei elicited mainly an excitatory response in the pineal gland of guinea pigs (Semm et al., 1981). One study also found

\footnotetext{
${ }^{1}$ The number of units investigated in this study appears erroneous. This may possibly be due to a rounding error by the authors.
}

approximately one-quarter (26.9\%) of spontaneously discharging cells tested to be unresponsive to SCG stimulation, indicating these cells may respond to innervation from other central or peripheral sources (Reuss et al., 1985b). This supports possible somatosensory and central inputs to the pineal gland, although further investigation to the exact nature of this input remains to be carried out.

Several authors (Reuss et al., 1985b; Reyes-Vazquez et al., 1986; Stehle et al., 1987) also note the presence of "silent units" within the pineal, in which no spontaneous electrical activity was initially observed. However, following stimulation, these silent cells showed discharge patterns similar to the spontaneously active cells. These "silent cells," first described by Brooks et al. (1975) and later by Reyes-Vazquez et al. (1986), have also been observed following stimulation of the habenular nuclei (Ronnekleiv et al., 1980; Reuss et al., 1984). This indicates that these cells likely only fire in response to deliberate input from structures contributing the pineal gland innervation, therefore, exhibiting no spontaneous input of their own. However, the exact function of these cells remains unknown. Therefore, further studies are required in order to uncover the purpose of these silent cells and the nature of their firing.

The experiments by Reuss et al. (1985b) indicate that certain pinealocytes exhibit a preferential response to specific forms of input from the SCG (see Figure 4). Further, the authors note a small number $(7.7 \%)$ of the cells were either inhibited or augmented in their electrical discharge depending on whether stimulation was arising from the right or left ganglion, respectively, with their response following bilateral stimulation being unclear. These experiments show that sympathetic innervation of the pineal gland is not simply a case of recruiting excitation of all pinealocytes to the same degree, and that some pinealocytes serve different roles upon receiving sympathetic innervation. Whilst an excitatory response may facilitate synthesis of melatonin, an inhibitory response may prevent this. This could be mediated via astrocytes in close proximity to the pinealocytes releasing glutamate (Villela et al., 2013) or GABA (Minchin and Iversen, 1974), or even neighbouring pinealocytes releasing GABA, as both neurotransmitters are known to decrease melatonin synthesis (Rosenstein et al., 1989). Indeed, the presence of silent-cells that only respond to input from a specific structure support the notion of input-specific response pinealocytes (Ronnekleiv et al., 1980; Reuss et al., 1984, 1985b; Stehle et al., 1987). With this in mind, perhaps such response preferences of pineal cells exist but impart equal output, thus facilitating no overall difference in the magnitude of the total responses between unilateral and bilateral stimulation. It does appear that simultaneous input from both SCG is not additive (Patel and Demaine, 1990), and therefore the pineal cellular response of bilateral stimulation is not any greater than that of unilateral stimulation. This suggests that the cells recruited to fire following bilateral SCG stimulation are not the same populations recruited for unilateral stimulation, or are recruited to a lesser degree. It is also possible that interspecies differences between the rat and the hamster could be responsible for such inconsistencies in these findings. Finally, variation in stimulation frequencies used could also account for such 
differences. Frequency disparities include: $1 \mathrm{~Hz}$ (Reyes-Vazquez et al., 1986), 10-20 Hz (Patel and Demaine, 1990), whilst one did not describe the frequency used (Pazo and Gonzalez, 1991). Such differences in results may highlight the importance of the stimulation frequency used for such experiments.

The above studies show that modulation of the pineal gland is achievable on a cellular level, however, they also highlight the variability in response rate that can occur with different stimulation paradigms. It appears that significant thought should be given to the surgical approach and site(s) of invasive stimulation as well as the frequency and pulse durations used when attempting to exert a maximal modulatory cellular response in the gland.

\section{Invasive Stimulation: Indoleamine Output and Enzymatic Activity of the Pineal Gland}

Data obtained from single cell recordings in the pineal gland are important. However, knowledge of how stimulation of relevant anatomical structures impacts the indoleamine and enzymatic output of the gland provides more clinically translatable information. Such output of the pineal gland as a result of electrical stimulation of the sympathetic innervation pathway has been researched and will subsequently be summarised below.

The enzymatic activity of the pineal following stimulation of either the pre- or postganglionic neurons of the SCG was investigated (Bowers and Zigmond, 1982). Stimulation was delivered for $1 \mathrm{~h}$ at various frequencies. In both conditions, stimulation from $5-10 \mathrm{~Hz}$ produced maximal upregulation of AANAT activity when differences in length of time animals were in surgery and exposed to light were accounted for. Interestingly, stimulation at $1 \mathrm{~Hz}$ elicited a decrease in AANAT activity following pre-ganglionic stimulation, whereas AANAT activity remained unchanged following postganglionic stimulation at the same frequency. The authors offer that such a difference may be due to failure of synaptic transmission between pre- and postsynaptic neurons in the ganglia at low frequencies. The fact that no differences in AANAT activity was observed between preand postganglionic stimulation conditions at higher frequencies indicates that it is the lower frequency $(1 \mathrm{~Hz})$ enabling this transmission failure.

Volkman and Heller (1971) were amongst the first to demonstrate in vivo changes in rat pineal AANAT levels following direct, invasive stimulation of structures involved in the pineal sympathetic pathway during the day. Unilateral stimulation (10 $\mathrm{Hz}$ for $9 \mathrm{~s}$ every min for $1-3 \mathrm{~h}$ ) of the pre-ganglionic CST was performed, which resulted in a duration-dependent increase in AANAT levels compared to control groups.

Following light-induced reduction of AANAT levels in vivo in rats, stimulation of the CSTs at $5 \mathrm{~Hz}$ during the night increased pineal AANAT levels in a linear fashion greater than what is observed during the night (Bowers and Zigmond, 1980). Volkman and Heller (1971) saw a lesser increase following their stimulation experiments. The latter stimulated the CSTs during the daytime, whereas the former stimulated during the night, which is claimed key to observing an immediate increase in AANAT levels comparable to those reached during the nightly peak (Bowers and Zigmond, 1980). It's postulated that stimulation during the daytime incurs a time delay in the physiological response of the pineal either to darkness, pharmacological stimulation, or electrical stimulation of the darkness signalling pathway (Bowers and Zigmond, 1980). However, it was later demonstrated that it is possible to maximally upregulate AANAT levels via stimulation during the day, disputing this theory (Bowers et al., 1984a). Following cessation of stimulation, Bowers and Zigmond (1980) also observed a rapid decline of pineal AANAT levels with a half-life of approximately $5 \mathrm{~min}$, similar to that observed following exposure to light or administration of propranolol - a beta blockers that dampens sympathetic activity and, therefore, upregulation of AANAT. This indicates that continual stimulation would be required in order to maintain high pineal output for any significant length of time.

Bilateral stimulation of the SCG in rats ex vivo significantly elevated pineal cAMP levels compared to sham-stimulated controls (Heydorn et al., 1981). cAMP is a crucial component of the second messenger system mediating the upregulation of AANAT and is itself upregulated via NE released from postganglionic sympathetic terminals. Therefore, an increase in cAMP will result in an increase in AANAT, which facilitates the synthesis of melatonin. Although various stimulation parameters were investigated, those optimal for increasing cAMP levels were: $10 \mathrm{~Hz}, 20 \mathrm{~V}$ with a pulse duration of $10 \mathrm{~ms}$ for $1 \mathrm{~min}$. The authors also found that this cAMP increase could be potentiated by more than 4 -fold via the prior administration of desmethylimipramine - a tricyclic antidepressant that prevents reuptake of catecholamines including NE into sympathetic nerve terminals. This indicates that a surplus of NE allows for further increases in cAMP levels, however, it is not confirmed that such a surplus translates into greater increases in AANAT or melatonin levels.

Bowers and Zigmond (1982) compared the effects of bilateral stimulation of the rat CSTs in both day-time and night-time conditions at different frequencies. Prior to stimulation, exposure to light was used to reduce AANAT levels to levels encountered during the day. Stimulation for $2 \mathrm{~h}$ during the night-time at frequencies of $5-10 \mathrm{~Hz}$ induced maximal linear increases in AANAT activity. Stimulation at $2.5 \mathrm{~Hz}$ during the night increased AANAT levels linearly up to a point of $30 \mathrm{~min}$ before plateauing for the next $90 \mathrm{~min}$. When elevated AANAT levels were achieved by $1 \mathrm{~h}$ of high frequency stimulation, stimulation at $2.5 \mathrm{~Hz}$ proved insufficient in maintaining these increased AANAT levels. Whilst stimulation frequencies of $2.5 \mathrm{~Hz}$ and above induced an increase in AANAT activity, stimulation at $1 \mathrm{~Hz}$ produced a significant decrease in AANAT activity. Such results support the notion of frequency-dependent modulatory effects, although it is surprising that the point at which this effect switches from one of downregulation to upregulation is as low as $2.5 \mathrm{~Hz}$.

Bowers and Zigmond (1982) stimulated at $10 \mathrm{~Hz}$ during the day, AANAT activity significantly increased during the first hour of stimulation but at a reduced rate compared to stimulation during the night-time. However, the rate of increase was significantly elevated with each passing hour of stimulation. This indicated that longer periods of stimulation facilitate greater increases in AANAT activity. Similar results were later reported 
showing tripling of AANAT activity upon bilateral stimulation of the SCG at $10 \mathrm{~Hz}$ for $2 \mathrm{~h}$ during the day-time (Reuss et al., 1989). This was further confirmed when stimulation of the CSTs during the light period at $10 \mathrm{~Hz}$ for $3 \mathrm{~h}$ produced levels similar to that of peak night-time AANAT levels (Lingappa and Zigmond, 2013). However, Reuss et al. (1985b) did not find any significant change in enzyme levels following day-time stimulation at $10 \mathrm{~Hz}$ or $25 \mathrm{~Hz}$ for $15 \mathrm{~min}$. This shows that stimulation periods must exceed $15 \mathrm{~min}$ in order to accommodate any significant elevation in AANAT activity.

Stimulation frequencies of 2.5 and $5 \mathrm{~Hz}$, but not $1 \mathrm{~Hz}$, were reported as capable of achieving submaximal AANAT levels to that seen during the night-time (Lingappa and Zigmond, 2013). This is in accordance with previously reported findings (Bowers and Zigmond, 1982). Taken together, findings suggest that whilst stimulation of the CSTs or SCG during the day-time at $10 \mathrm{~Hz}$ for $3 \mathrm{~h}$ can elicit significant upregulation of AANAT, stimulation during the night-time at a minimum frequency of $5 \mathrm{~Hz}$ for at least $2 \mathrm{~h}$ is optimal.

The effects of bilateral and unilateral stimulation of the CSTs using $10 \mathrm{~Hz}$ stimulation for $1 \mathrm{~h}$ at night were also examined (Bowers and Zigmond, 1982). Whilst there was no difference in AANAT activity as a result of left vs right CST unilateral stimulation, bilateral stimulation during the nighttime produced a greater increase ( $>3$-fold) in AANAT levels compared to unilateral stimulation. This suggests that there is equal input from both ganglia to the pineal, and bilateral CST stimulation is necessary in order to drive a maximal pineal sympathetic response.

Changes in the enzyme HIOMT in response to stimulation of structures in the pineal sympathetic pathway have not been fully investigated. This is likely because $\mathrm{N}$-acylation of serotonin by the enzyme AANAT is generally considered to be the rate-limiting step in melatonin biosynthesis. Contrarily, it has been hypothesised that HIOMT is the true ratelimiting step in the pathway (Liu and Borjigin, 2005). This is due to high levels of pineal melatonin being apparent during the dark phase in rats despite chronic low levels of AANAT as a result of a point mutation in the Aanat gene. Following stimulation of the pre-ganglionic SCG fibres, one study (Reuss et al., 1989) observed no changes in the activity levels of the enzyme HIOMT when stimulating for $2 \mathrm{~h}$, whereas another previously reported an $18 \%$ decrease in HIOMT when stimulating for $4 \mathrm{~h}$ (Brownstein and Heller, 1968). It is possible that this difference is due to insufficient stimulation time, yet, it has been previously shown that $2 \mathrm{~h}$ of stimulation is sufficient to maximally upregulate AANAT levels (Bowers and Zigmond, 1982). Such differences in results may be due to variations in stimulation paradigms and/or experimental protocols. Further differences pertaining to these two studies include enucleated vs blinded animals, unilateral vs bilateral stimulation, stimulation current, stimulation pulse duration, and type of anaesthetic used (for further details please see Table 2).

Most studies into pineal neuromodulation have been conducted in rats that are nocturnally active creatures. Mice appear to be disfavoured among studies investigating pineal output due to the fact that many commonly used strains of laboratory mice do not produce melatonin (Goto et al., 1989). Unilateral stimulation of the left SCG during the light period in the rabbit produced a significant increase (on average 15-fold) in plasma melatonin levels compared to pre-stimulation levels (Chan et al., 1989). Rabbits were stimulated at an unusually high frequency $(300 \mathrm{~Hz})$ at $5 \mathrm{~mA}$ for $7.5 \mathrm{~s}$ every $20 \mathrm{~s}$ for $24-60 \mathrm{~min}$. Blood samples were collected from the confluens sinuum, where it is generally agreed that pineal melatonin is secreted (Quay, 1973). This means it is reasonable to assume the levels of plasma melatonin detected are not, in fact, secreted from extrapineal sources. As rabbits are crepuscular rather than nocturnal, such a study shows pineal output alteration can be achieved in different species despite their varying sleep/wake rhythms. The authors use of such a high frequency is curious as the other experiments reviewed do not use frequencies any higher than $80 \mathrm{~Hz}$. Moreover, it has been previously shown that repetitive stimulation of the preganglionic SCG fibres at frequencies higher than $35 \mathrm{~Hz}$ in the rabbit results in a decline in successive action potential amplitude (Eccles, 1955). This suggests that although a pineal modulatory response may be elicited with stimulation at $300 \mathrm{~Hz}$, a maximal response may be achieved with a much lower frequency.

The pattern of stimulation was an important determinant in the upregulation of AANAT despite the same average stimulation frequency (Bowers and Zigmond, 1982). 2-s bursts of $10 \mathrm{~Hz}$ bilateral CST stimulation every $20 \mathrm{~s}$ was amongst the most proficient to increase enzyme levels in comparison to constant $1 \mathrm{~Hz}$ stimulation for the same length of time (Bowers and Zigmond, 1982). This agrees with previous findings that documented successive episodes of bilateral stimulation produced a larger overall response from pineal cells compared to a single episode (Reuss et al., 1985b). In addition, it confirms that $1 \mathrm{~Hz}$ is incapable of upregulating pineal indoleamine/enzyme levels. However, it was found that $1 \mathrm{~s}$ bursts of $10 \mathrm{~Hz}$ every $10 \mathrm{~s}$, and $4 \mathrm{~s}$ bursts of $5 \mathrm{~Hz}$ every $20 \mathrm{~s}$ did not significantly increase enzyme levels. Together, these findings indicate that whilst repeated episodes of bilateral stimulation at a frequency greater than $5 \mathrm{~Hz}$ are most effective for increasing sympathetic pineal output, the specific pattern of stimulation is important in order to exert an effect.

Fibres projecting from the SCN to the PVN are thought to be gamma-aminobutyric acid (GABA)-ergic in their output, ultimately resulting in inhibition of pineal melatonin synthesis (Teclemariam-Mesbah et al., 1999; Kalsbeek et al., 2000; Buijs et al., 2003). This aligns with the identity of the SCN as the master pacemaker, which exerts control over slave oscillators such as the pineal gland. The functional output of fibres arising from PVN is less clear. It has been previously speculated that the PVN provides inhibitory input to the pineal sympathetic pathway and experimental data at the time seemed to support this hypothesis (Gilbey et al., 1982a,b). However, recently the $\mathrm{PVN}$ is thought to be responsible for communicating excitatory glutamatergic signals to the IML (Yanovski et al., 1987; Kannan et al., 1989). Therefore, stimulation of the PVN is expected to result in an increase in pineal metabolic output. Variation of bilateral stimulation duration $(10 \mathrm{~Hz}$ at $0.1 \mathrm{~mA}$ for $0.2 \mathrm{~ms})$ of 
the PVN resulted in a significant reduction in pineal AANAT levels following $60 \mathrm{~min}$ of PVN stimulation in rats during the day-time (Reuss et al., 1985a). During the night-time, a minimum of 30 min stimulation was necessary in order to significantly reduce AANAT levels, however, neither AANAT nor melatonin levels were reduced to that of day-time levels, but perhaps longer lengths of stimulation are necessary in order to elicit such results. Likewise, it was demonstrated that brief ( $2 \mathrm{~min}$ ) invasive bilateral electrical PVN stimulation $(10 \mathrm{~Hz})$ is capable of inducing significant decreases in pineal melatonin, AANAT and NE in rats, compared to control levels - similar to the effect achieved with exposure to light during the night-time (Olcese et al., 1987). Given that the PVN is thought to provide excitatory input to the IML, this decrease in AANAT levels is somewhat unexpected. However, it has been previously noted that urethane anaesthesia can trigger a decrease in sympathetic output of the PVN (Kannan et al., 1987, 1989; Yamashita et al., 1987). Both Reuss et al. (1985a,b) and Olcese et al. (1987) used urethane as anaesthetic during their stimulation experiments, therefore it is possible that such use inflicted a confounding effect on their results, masking an otherwise excitatory sympathetic outflow of the PVN.

Placing potential confounding influences aside, Olcese et al. (1987) deduced that the observable changes in AANAT, melatonin and NE levels after such short stimulation periods were due to waiting at least $30 \mathrm{~min}$ before sacrifice. Previous experiments that euthanized animals immediately following cessation of the stimulation period reported significant changes in pineal AANAT, melatonin and NE levels only after much longer periods of stimulation (e.g., Reuss et al., 1985b). Therefore, they inferred that there exists a time delay between application of a stimulus and an effect on pineal melatonin and AANAT levels (Olcese et al., 1987). Whilst this may ring true for the mechanisms involved in decreasing pineal AANAT and melatonin levels, other experiments (Volkman and Heller, 1971) indicate this is not the case for inducing increasing pineal AANAT levels. In fact, it was found that AANAT levels were lower when waiting an hour poststimulation prior to sacrificing as opposed to levels encountered upon immediate sacrifice.

\section{Non-invasive Stimulation}

Alongside experiments that directly stimulate structures in the pineal sympathetic innervation pathway, non-invasive stimulation methods have also been explored. The effects of unilateral transcutaneous auricular vagus nerve stimulation on pineal melatonin levels in Zucker lean and Zucker diabetic obese (ZDO) rats was investigated (Li et al., 2014). Stimulation was applied once during the day-time to the right auricular concha region via opposing magnetic electrodes. Stimulation frequency switched every second between 2 and $15 \mathrm{~Hz}$ at a current of $2 \mathrm{~mA}$. Following 34 consecutive days of stimulation, plasma melatonin levels in stimulated ZDO rats was significantly higher compared to non-stimulated controls. Furthermore, this elevated concentration was detectable for over $20 \mathrm{~h}$ after the final stimulation session. Notably, the experimenters investigated the effect of transauricular vagus nerve stimulation (taVNS) on pinealectomised ZDO rats and were still able to observe acute increases in plasma melatonin levels similar to that seen in intact ZDO rats. Similar results were also achieved 1 year later using bilateral taVNS (Wang et al., 2015). As increases in melatonin were still observed despite removal of the pineal gland, this indicates that vagus nerve stimulation prompts secretion of melatonin from extrapineal sites rather than from the gland itself. However, since usual pinealectomy removes only the superficial portion of the gland, leaving the deep pineal intact, one cannot discount the possibility that circulating melatonin levels may be due to contribution from the deep portion of the gland. Yet, atrophy of the deep pineal is apparent following superficial pinealectomy in rats (Heidbuchel and Vollrath, 1983), which most likely results in impaired function, although this has not been confirmed. This is not surprising since the sympathetic fibres that innervate the gland first supply the superficial pineal before coursing down the stalk and supplying innervation to the deep pineal. This suggests that removal of the superficial pineal disrupts the sympathetic input to the deep pineal, which would account for the observed atrophy. If sympathetic input is disrupted, then one may speculate that the likelihood of the deep pineal contributing to systemic melatonin levels is low.

Studies into the antidepressant activity of electroconvulsive shock (ECS) therapy and its relation to pineal hormone levels are a less specific form of non-invasive pineal neuromodulation. No significant changes in pineal melatonin nor NAS levels following such stimulation were observed in rats (McIntyre and Oxenkrug, 1984). However, a significant decrease (40\%) in serotonin levels in the pineal gland following seven days of single electroconvulsive stimulation at $130 \mathrm{~V}$ for $0.75 \mathrm{~s}$ was shown. Contrasting findings have been reported. Delivery of an ECS (130 V administered for $0.75 \mathrm{~s}$ ) also via ear clip electrodes to rats during the day-time in order to induce tonic-clonic seizures lasting between $20-25$ s resulted in pineal melatonin and serotonin levels had doubling, and 5-HIAA levels increased by $80 \%$ (Oxenkrug et al., 1991). However, NAS levels were below the detection limits in both groups, a finding that authors speculate could be due to all available NAS being rapidly converted into melatonin. Interestingly, serotonin levels were not significantly changed following stimulation, which contrasts with the previous findings (McIntyre and Oxenkrug, 1984). Given that both studies utilised the same stimulation parameters, the reasons underlying these differences remain unclear. It is possible that variation in experimental procedures are accountable. For example, Oxenkrug et al. (1991) extracted pineals for analysis $90 \mathrm{~min}$ following the cessation of stimulation whereas McIntyre and Oxenkrug (1984) performed extraction at $2100 \mathrm{~h}$ despite stimulating in the morning. This prolonged delay between stimulation and pineal extraction may have caused any pineal indoleamine increase to have dissipated. Consistent with this, invasive studies discussed in the above sections reinforce the notion that pineal modulatory effects are short-lived following cessation of the stimulus (Volkman and Heller, 1971; Bowers and Zigmond, 1980).

Nowak et al. (1988) examined AANAT levels following administration of trains of 10 ECS via ear clip electrodes to rats. Each train lasted $500 \mathrm{~ms}$ at a frequency of $50 \mathrm{~Hz}$, and was delivered daily for ten consecutive days. No significant differences 
were found in pineal AANAT levels in the experimental group following the course of stimulation compared to the control group. The authors also investigated the effect of ECS coupled with the $\beta$-adrenoreceptor agonist, isoproterenol. It was shown that treatment with this agonist in conjunction with a single ECS during the day-time produced a significant increase in AANAT compared to isoproterenol treatment alone. This increase was only apparent when tissue was collected $4 \mathrm{~h}$ after treatment onset, whereas no increase was observed when tissue was collected after $2 \mathrm{~h}$. This is in contrast to findings that suggest a minimal delay between stimulation and analysis of the gland's contents is optimal for detection of pineal substances of interest (Volkman and Heller, 1971; Bowers and Zigmond, 1980; McIntyre and Oxenkrug, 1984; Oxenkrug et al., 1991). Interestingly, when isoproterenol was coupled with trains of ten ECSs over 10 days, no significant change in AANAT levels were observed compared to the treatment with the agonist alone, regardless of euthanasia time. This indicates that a single stimulation period is capable of upregulating enzyme levels but the mechanism by which this occurs takes over $2 \mathrm{~h}$ to yield a significant effect. It also suggests repeated sessions are not capable of modulating the enzymatic activity of the pineal gland. This may be due to saturation of the enzyme induction mechanism responsible for the upregulation of AANAT leading to enzyme depletion, desensitisation of the adrenergic receptors, or perhaps some form of negative feedback circuit that prevents excessive Aanat gene expression such as receptor downregulation. Such an explanation could provide clarification as to why McIntyre and Oxenkrug (1984) also found no significant modulation of melatonin nor NAS levels with repeated stimulation, whereas Oxenkrug et al. (1991), using single instances of stimulation, did.

The studies utilising ear clip electrodes may be stimulating the vagus nerve rather than the pineal sympathetic innervation pathway. This is because an auricular branch of the vagus nerve exists in the ear and stimulation of this part of the anatomy has been shown to successfully stimulate the vagus nerve (Lewy and Newsome, 1983; Kreuzer et al., 2012, 2014; Capone et al., 2015). Two studies explored this possibility by investigating plasma melatonin levels in both intact and pinealectomised rats (Li et al., 2014; Wang et al., 2015). It was found in both cases that increases in the hormone were still evident in the pinealectomised groups indicating melatonin release is facilitated from extrapineal sources. Nowak et al. (1988) did not investigate the effects in pinealectomised rats, however, AANAT levels were quantified following homogenisation of the pineal gland, confirming increased levels of the enzyme in the gland.

The release of melatonin has been suggested to be an endogenous, anticonvulsant (for review see Munoz-Hoyos et al., 1998). Indeed, studies have investigated this line of research in addition to exploring therapeutic interventions that aim to increase endogenous melatonin. Chao et al. (2001) induced seizures via injection of benzylpenicillin into the hippocampus of rats followed by administration of electroacupuncture to the acusites Fengfu (DU16) and Jinsuo (DU8). These acusites are located on the midline at the nape of the neck and between the ninth and tenth thoracic vertebrae, respectively. According to a dermatome mapping study, the nape of the neck is related with spinal nerves $\mathrm{C} 2$ and $\mathrm{C} 3$ in the rat (Takahashi and Nakajima, 1996). Following electroacupuncture, both central and peripheral melatonin levels were shown to be significantly elevated. At the level of C2 and C3, sympathetic input is supplied by the SCG, which is implicated in the pineal sympathetic innervation pathway (Netter, 1999; von Lanz and Wachsmuth, 2003; Lingford- Hughes and Kalk, 2012). Therefore, it is possible that electroacupuncture at this site stimulated this pathway directly to increase pineal metabolic output. Acupuncture increased nocturnal levels of the urinary melatonin metabolite 6-sulfatoxymelatonin (a6MTs) following ten bi-weekly acupuncture sessions in humans (Spence et al., 2004). In addition, increased nocturnal levels of the same urinary metabolite were found following a 5 -week intervention consisting of two acupuncture treatments per week (Kayumov et al., 2003). Both studies do not note the acusites employed during the experiments, therefore no deduction can be made on which dermatomes were stimulated in order to facilitate increase in a6MT levels. Moreover, there is a possibility the myotomes or osteotomes were stimulated rather than dermatomes, which could exert different effects than those intended. Also, it is possible that the increase in the a6MT metabolite may be due to release of melatonin from extrapineal sites. In disagreement with this, a decrease in metabolite levels occurs in both humans (Neuwelt and Lewy, 1983) and rats (Lewy et al., 1980) following pinealectomy, supporting the notion of the pineal gland as the major source of circulating melatonin. Although these results support the idea that neuromodulation of pineal gland metabolic output may be achieved by less invasive stimulatory methods, further studies are required - perhaps utilising pinealectomy - in order to clarify whether increased melatonin is due to stimulation of the pineal gland and not other structures.

\section{CLINICAL ASPECTS}

\section{Melatonin}

Melatonin is a circadian rhythm synchroniser (Pfeffer et al., 2018) and disruptions in melatonin levels are linked to sleep disorders and chronic sleep deprivation (Zisapel, 2018). Sleep deprivation is a pervasive problem throughout society. A wide variety of reasons contribute to this problem such as: stress, prevalence of shift work, and prolonged working hours due to the advent of artificial light. In the short-term, sleep deprivation is associated with negative effects on memory, psychomotor skills, attention, and hyperalgesia (Zeitlhofer et al., 2000; Hoevenaar-Blom et al., 2011; Krause et al., 2019). Sleep deprivation is associated with a number of negative health repercussions, including suppression of the immune system (Dinges et al., 1995), acceleration of atherosclerosis (McAlpine et al., 2019), increased risk of developing obesity (Cappuccio et al., 2008), and development of certain cancers (Blask, 2009) (e.g., breast, Hansen, 2001; Mirick et al., 2001; Schernhammer et al., 2001, 2006), prostate (Kubo et al., 2006), endometrial (Viswanathan et al., 2007), and colorectal cancer (Schernhammer et al., 2003). In addition, one night of acute sleep deprivation is sufficient to increase DNA damage in otherwise healthy young adults (Cheung 
et al., 2018). DNA damage, particularly double strand breaks, are especially hazardous to the genome. Disrepair upon replication leads to cell death, whilst misrepair can result in inappropriate end-joining events, which commonly underlies tumour development. As melatonin is a potent free-radical scavenger and powerful combatant against oxidative stressinduced damage, maintenance of optimal melatonin levels may be protective against DNA damage. This may be achieved through one or several of melatonin's known protective pathways such as: inhibiting pro-oxidative enzymes, activating antioxidant enzymes, and/or promoting DNA repair mechanisms.

Melatonin's antioxidant, anti-inflammatory, and free-radical scavenging properties may also be harnessed to promote tissue regeneration. When delivered via biomaterials that control its release, melatonin accelerates wound repair (Murali et al., 2016), promotes metabolic activity and proliferative capacity of mesenchymal stem cells (Çetin Altındal et al., 2019; Hu and Li, 2019), and enhances peripheral nerve regeneration (Qian et al., 2018). These actions are exerted through melatonin's regulation of the microenvironment and its antioxidant and anti-inflammatory properties. Melatonin and NAS also act as neuroprotective agents via several mechanisms (Luo et al., 2019) including: combating oxidative stress (Wang et al., 2009; Chern et al., 2012; Li et al., 2014; Wong et al., 2014), inhibiting cell death processes (Savaskan et al., 2007; Wang et al., 2009; Zhang et al., 2013; Zhou et al., 2014; Hoshino et al., 2017), and promotion of anti-inflammatory pathways (Wang et al., 2009, 2013; Carloni et al., 2016; Lin et al., 2016). Such findings indicate melatonin as a promising focus in regenerative and therapeutic medicine with potential benefits for sufferers of stroke, traumatic brain injury, and neurodegenerative diseases.

Sleep deprivation is also linked to an increased risk of developing neurodegenerative diseases such as Alzheimer's disease (AD) (for review see: Kumar and Chanana, 2014). It has been demonstrated via sampling CSF, that merely one night of sleep deprivation is sufficient to interfere with the normal physiological drop in $\beta$-amyloid $(\mathrm{A} \beta)$ protein levels (Ooms et al., 2014) and that disruption to slow-wave sleep is key to this effect (Zempel et al., 2017). This suggests that chronic slow-wave sleep deprivation may result in continuous elevation and accumulation of $\mathrm{A} \beta$, which is theorised as the starting point for $\mathrm{AD}$ pathogenesis according the amyloid cascade hypothesis (Hardy and Higgins, 1992). Moreover, animal studies indicate that disruption to the sleep-wake cycle promotes excessive hyperphosphorylation of the tau protein in the brain (Rothman et al., 2013; Di Meco et al., 2014) leading to the formation of neurofibrillary tangles (NFTs), which are considered a neurological hallmark of AD. Interestingly, levels of melatonin in the CSF of AD patients progressively diminish as the disease progresses (Liu et al., 1999). Given that melatonin is a prominent regulator of the sleep-wake cycle, it has been suggested that this reduction in melatonin may at least partially drive the development of the disease. In this context, upregulation of endogenous melatonin may help slow the progression of AD. Indeed, melatonin has been shown to have beneficial effects both pre- and post- $\mathrm{A} \beta$ formation through transcriptional regulation of A $\beta$ synthesis (Peng et al., 2013; Panmanee et al., 2015;
Shukla et al., 2015; Mukda et al., 2016), acting as an antioxidant and free-radical scavenger to combat oxidative stress associated with $\mathrm{A} \beta$-induced neurotoxicity (Rodriguez et al., 2004; Reiter et al., 2013; Zhang and Zhang, 2014) and, inhibition of $\mathrm{A} \beta$ fibrillogenesis (Pappolla et al., 1998; Bazoti et al., 2005) (for review see: Vincent, 2018).

Some sleep disorders are considered prodromal markers for such neurodegenerative diseases. For example, REM sleep behavior disorder (RBD) is a parasomnia that is characterised by an absence of muscle atonia during REM sleep. This results in sufferers acting out their dreams in a vigorous and often violent manner. RBD is strongly linked with $\alpha$-synucleinopathies, and is considered a prodromal marker for dementia with Lewy Bodies, multiple system atrophy, and Parkinson's disease (Boeve et al., 2013). Melatonin is known to influence REM sleep latency and length (Cajochen et al., 1997; Kunz et al., 2004), and exogenous melatonin is currently used as a symptomatic treatment for RBD with clinical and neurophysiological benefits still observed up to 3 years following cessation of treatment (Kunz and Bes, 1999; Boeve et al., 2003; Kunz and Mahlberg, 2010; Schaefer et al., 2017). Further, as endogenous levels of melatonin decrease with age (Waldhauser et al., 1988; Garfinkel et al., 1995), which coincides with an increase in neurodegenerative disorders, it is possible that the age-associated decline in melatonin levels is causally linked to the development of neurodegenerative diseases (Reiter et al., 1994). Therefore, stimulating an increase in endogenous levels of melatonin may have beneficial protective, antioxidant and free-radical scavenging effects to dampen the progression of neurodegenerative diseases.

\section{NAS}

$\mathrm{N}$-acetylserotonin, being an immediate precursor to melatonin and also showing circadian rhythmicity (Chattoraj et al., 2009), has naturally been investigated for its potential effects on sleep regulation. However, recent research is examining the role of NAS on neurogenesis, mood regulation and neuroprotection. Neurogenesis occurs throughout adulthood in humans and rats in the dentate gyrus of the hippocampus as well as along the rostral migratory stream (Eriksson et al., 1998; Gould et al., 1999; Curtis et al., 2007). Studies have shown voluntary exercise and environmental enrichment can increase neurogenesis, whereas both aging and sleep deprivation impair the neurogenic response (Ming and Song, 2005; Lledo et al., 2006; Zhao et al., 2008; Ma et al., 2009). It is possible that exogenous administration of NAS could play a role in stimulating an increase in neurogenesis, and therefore, infer a positive influence on aspects of memory, mood control, and mitigate the development of neurological conditions.

Early studies have found NAS and its associated enzyme, AANAT, present in areas of the CNS such as the hippocampus, olfactory bulb, spinal cord and cerebellum despite the absence of melatonin (Paul et al., 1974; Psarakis et al., 1982; Gaudet et al., 1991; Chae et al., 1999). Further, approximately 15\% of synthesised melatonin is converted back into NAS (Leone and Silman, 1984; Young et al., 1985). NAS also shows a much higher affinity for the MT3 receptor than melatonin itself Nosjean et al. (2000) leading to some researchers pondering 
whether the MT3 receptor should actually be reclassified as a NAS receptor (Jang et al., 2010; Oxenkrug and Ratner, 2012). Further, unlike melatonin, NAS is able to activate the tropomyosin receptor kinase $\mathrm{B}$ ( TrkB) receptor, which is involved in mediating the effects of the neurotrophic factor, BDNF (brain derived neurotrophic factor) that plays a role in regulating neuronal activity and normal day-to-day function (Jang et al., 2010). BDNF-TrkB signaling is known to regulate a wide range of functions including: cell survival, neuronal differentiation and migration, neurite outgrowth, and facilitation of longterm potentiation and plasticity. Both BDNF and its high affinity receptor, TrkB, are widely produced throughout the CNS with high expression being observed in areas such as the neocortex, hypothalamus, and amygdala. Similar to melatonin, the expression of both BDNF and TrkB decrease with age, indicating neurons and glia yield a limited trophic ability to combat natural and pathological neurodegeneration (Berretta et al., 2014). In the absence of BDNF, NAS may act as an agonist for the TrkB receptor and enable circadian rhythmicity and also stimulate neurogenesis (Jang et al., 2010). Further, it seems that NAS is able to protect against sleep-deprivationinduced suppression of neurogenesis (Sompol et al., 2011). Whilst melatonin has previously been implicated in hippocampal neurogenesis (Ramirez-Rodriguez et al., 2009, 2011; Rennie et al., 2009; Manda and Reiter, 2010), further research indicates it does not increase the number of neuroblasts (Jang et al., 2010; Sompol et al., 2011), but may instead assist in neuroblast survival (Ramirez-Rodriguez et al., 2011). Taken together, these findings strongly indicate a melatonin-independent neuroregenerative role for NAS. Moreover, they suggest NAS is sufficient to induce hippocampal neurogenesis and mediate the negative effects chronic sleep deprivation imposes on hippocampal neurogenesis. Therefore, upregulation of pineal metabolic output may infer therapeutically beneficial effects via increasing systemic NAS levels in addition to melatonin levels. Given the interaction of NAS with TrkB, it remains to be determined if NAS-TrkB signalling has anti-brain aging and enhanced brain plasticity effects, similar to BDNF-TrkB signaling. The physiological and pathophysiological effects of NAS still remain poorly understood and much further work is required to investigate the role NAS plays in normal brain function.

\section{EVALUATION OF STIMULATION PARAMETERS}

\section{Frequency}

The frequency of stimulation appears to be particularly important when attempting to maximally influence pineal gland activity. A $10 \mathrm{~Hz}$ frequency appears capable of eliciting temporal facilitation whilst much lower frequencies are not. This suggests higher frequency stimulation exerts a greater effect on pineal sympathetic activity compared to lower frequency stimulation. Indeed, this finding from evoked potentials appears to concur with findings investigating pineal metabolic output. Stimulation at $5-10 \mathrm{~Hz}$ elicits the greatest increases in AANAT activity, whilst $1-2.5 \mathrm{~Hz}$ stimulation elicit subdued responses (Bowers and Zigmond, 1982; Lingappa and Zigmond, 2013). Frequencies below $5 \mathrm{~Hz}$ seem to produce submaximal upregulation of pineal metabolism, with $1 \mathrm{~Hz}$ capable of downregulating AANAT activity. This downregulation may occur due to failure in synaptic transmission and only occur following stimulation of preganglionic fibres. A few studies investigated frequencies higher than $10 \mathrm{~Hz}$, however the frequency effects are unclear as the majority of the literature only specifies frequency range (i.e., 10-20 Hz). One study (Brooks et al., 1975) used a stimulation frequency of $30 \mathrm{~Hz}$ and successfully recorded evoked potentials in the pineal gland. This suggests higher frequencies are capable of influencing the gland electrophysiologically, however, it is unclear how this affects melatonin metabolism. Therefore, further research is required in order to examine the effects of high frequency invasive stimulation on pineal gland metabolic output.

From the reviewed studies, stimulation at $10 \mathrm{~Hz}$ appears to be the most efficacious frequency for invasive stimulation. Higher frequencies are more commonly used in non-invasive stimulation literature. However, detailed stimulation parameter reporting in these studies is generally poor with only half reporting the frequency of stimulation used. This means it is difficult to ascertain the effects of different stimulation paradigms in the context of non-invasive stimulation. Two studies that do report the stimulation frequency used offer conflicting results, with one indicating upregulation of melatonin levels, and the other only reporting upregulation of such levels in the presence of a $\beta$-adrenergic agonist. This could be due to differences in current used, or other experimental variables that are again, poorly defined. The evidence presented suggests non-invasive stimulation is capable of modulating pineal gland metabolic output but further studies are necessary before definite conclusions can be made on the optimal stimulation parameters to be used.

\section{Unilateral vs. Bilateral Stimulation}

It appears that whilst unilateral stimulation is capable of evoking changes in pineal gland output, bilateral stimulation produces a more pronounced effect. This is particularly evident in the study conducted by Bowers and Zigmond (1982) that describes unilateral stimulation producing less than half the increase in pineal AANAT activity compared to bilateral stimulation. This study provides a useful comparison of the effects between these two variables due to the conservation of experimental and reporting methods between experiments. It proves difficult to compare the effects of other studies as many utilise different measurement units for quantifying the levels of AANAT or melatonin as the weight of pineal glands are not often detailed, making it impossible to accurately compare such levels. Moreover, even when units between studies align, other parameters such as frequency, or stimulation length are not aligned, so direct comparisons of pineal levels cannot be made. Interestingly, there are no differences in evoked potentials from pineal cells between unilateral and bilateral stimulation (Patel and Demaine, 1990). This indicates that the observed pineal cell firing does not equate with the enzymatic upregulation in the grand in response to sympathetic input. 


\section{Duration of Stimulation}

The duration for which stimulation is administered appears to be critical. Stimulation for 15 min does not significantly increase AANAT levels, whereas stimulation for $2 \mathrm{~h}$ does (Reuss et al., 1989). This suggests that in order for stimulation at $5-10 \mathrm{~Hz}$ to produce optimal increases in AANAT activity, stimulation must be maintained for a sufficient length of time. Further, one study noted a decline in AANAT levels following cessation of $1 \mathrm{~h}$ at $5 \mathrm{~Hz}$ stimulation (Bowers and Zigmond, 1980). Another study reported that significant increases in AANAT activity persist following $2 \mathrm{~h}$ of stimulation at $10 \mathrm{~Hz}$ despite not sacrificing until $1 \mathrm{~h}$ following stimulation cessation (Volkman and Heller, 1971). This indicates that longer stimulation periods are likely to result in persisting pineal modulatory effects, whereas shorter periods of stimulation are not. Perhaps longer stimulation periods are required to alter AANAT levels due to transcription and translation of the Aanat gene being necessary before changes in the enzyme become apparent. Indeed, Nowak et al. (1988) reported that significant increases in AANAT levels were only apparent when sacrifice occurred $4 \mathrm{~h}$ following stimulation onset supporting the notion that such changes require some time before becoming apparent. In contrast, rapid changes in cAMP may be detected due to its relatively upstream position in the pineal sympathetic pathway. Rapid changes in pineal melatonin levels following PVN stimulation may be mediated via its theorised central input pathway to the gland, independent of the pathway incorporating the SCG.

\section{Repetition, Pulse Duration, and Pattern of Stimulation}

Successive bilateral SCG stimulation recruits a greater number of pinealocytes to fire, yet this is not the case for unilateral stimulation (Reuss et al., 1985b). This suggests a potential metamodulatory effect, but only when input is sourced from both ganglia. This again supports the notion of pinealocyte response heterogeneity that is dependent upon the source of input. However, as only a subset of cells within the pineal have been investigated thus far, it is perhaps not wise to extrapolate the results of such small cell populations to the response of the gland as a whole.

Pulse duration of the stimulus delivered to the CSTs appears to affect the current threshold necessary to generate an action potential. A strength-duration relationship is evident, with lower pulse durations requiring a greater current to be used in order to elicit an evoked response in the postganglionic fibres. The data suggest a minimum pulse duration of $3 \mathrm{~ms}$ is necessary in order to accommodate a lower current and prevent potential hyperpolarisation of postganglionic cell membranes.

The pattern of the stimulation pulse also appears important in the optimal upregulation of pineal AANAT (Bowers and Zigmond, 1982). Strangely, patterns that correspond to similar relative durations of "on" stimulation and "off" stimulation appear to have significantly different upregulatory powers. It is unclear why certain patterns are more capable of exerting an upregulatory influence than others. Perhaps a minimum pulse duration of $2 \mathrm{~s}$ is necessary and perhaps these optimal patterns reflect the endogenous stimulation patterns delivered to the pineal. However, a limited number of stimulation patterns have been investigated, therefore, a stimulation pattern of greater upregulatory effect may exist. Further studies are necessary in order to clarify the effects of various stimulation patterns on the response of the pineal gland.

\section{Light During Stimulation and Sacrifice}

Although pineal modulation appears to be possible at any time of the day, stimulation of the CSTs during the night elicits the most rapid response. Night-time stimulation linearly increases pineal AANAT activity - reaching peak night-time levels within $2 \mathrm{~h}$. In contrast, day-time stimulation has a variable rate of increase in AANAT activity, accelerating with passing time (Bowers and Zigmond, 1982). Stimulation of the PVN is equally effective in reducing AANAT levels when conducted during the day or night (Reuss et al., 1985a). However, such decreases during night-time stimulation are only significant when performed during the late portion of the dark phase (i.e., 0400-0600 h). This reduced effect of PVN stimulation does not seem to apply to stimulation of the CSTs, which proves capable of increasing AANAT activity during similar time periods (Bowers and Zigmond, 1980; Reuss et al., 1985b). It has been posited that the PVN provides inhibitory input to the pineal sympathetic pathway (Gilbey et al., 1982a,b). An explanation for this reduced response following PVN stimulation may be that inhibitory responses are themselves, in some way inhibited or dampened during the early and middle portions of the dark phase. Such dampening effects may help to maintain increased pineal indoleamine and enzyme levels during this time. However, more recently, the PVN is thought to be responsible for communicating excitatory glutamatergic rather than inhibitory GABA-ergic signals to the IML (Yanovski et al., 1987; Kannan et al., 1989). If this were true, one would expect PVN stimulation to increase AANAT levels. It has been previously noted that urethane anaesthesia can affect the sympathetic output of the PVN resulting in decreased sympathetic outflow (Kannan et al., 1987, 1989; Yamashita et al., 1987). Reuss et al. (1985a) used urethane anaesthesia during stimulation, therefore it is possible that such use inflicted a confounding effect on their results, masking an otherwise excitatory sympathetic outflow of the PVN.

Brief exposure to light during the dark phase is sufficient to rapidly decrease night-time AANAT levels to that of the day-time levels (Klein and Weller, 1972). It is therefore crucial that lighting conditions during night-time stimulations up to the point of sacrifice are carefully controlled. In the majority of the reviewed literature, the lighting conditions during such stimulation are not described. Lighting conditions during sacrifice are more often detailed as being carried out under dim-red light. Up until recently, dim-red "safe-light" with wavelengths above $600 \mathrm{~nm}$ was thought suitable for pineal-centred experiments due to the assumption that longer wavelengths of light exerted no influence on circadian rhythms. However, research in recent years strongly indicates that this is not the case. Dauchy et al. (2015) provided 
compelling evidence that plasma melatonin levels of rodents housed with such "safe-lights" during the night were significantly lower $(p<0.001)$ than those of rodents housed in complete darkness during the night. Such findings undermine the validity of the results of studies conducted using dim-red light. It is possible that levels of any pineal substances measured are not in fact the true maximal levels achievable either during the nightly peak or via stimulation. One way to circumvent such confounding factors may be to use complete darkness during the subjective night-time of the animals in future experiments. However, this may not prove practicable as manipulations often need to be carried out during the dark period and use of complete darkness would prove dangerous for both the researcher and animal due to increased risk of injury. Another approach is to blind the animals via optic transection or enucleation as some of the authors in this review have opted to perform. This would ensure the animals are not exposed to any light stimulus and allows the researcher to perform manipulations unimpeded. However, consideration must be given as to whether possible melatonin desynchrony, as occurs following blindness in humans (Lewy and Newsome, 1983), is allowable. Researchers may consider the use of infrared goggles to be able to see in the dark and not disrupt animals' melatonin levels. Some of these goggles do emit light, therefore care must be taken to perform manipulations quickly in order to ensure minimal disruption to melatonin rhythms.

\section{CONCLUSION}

The pineal neuromodulatory response is thought to be mediated via upregulation of the enzyme AANAT, which in turn facilitates the increase of melatonin via its aforementioned biosynthetic pathway. Previously, the majority of studies investigated the potential benefits of upregulating pineal melatonin levels. Melatonin is undoubtedly a hormone with many potential therapeutic benefits. Its reported influences on physiology are vast and well documented including: its actions as a potent antioxidant and free-radical scavenger (for review see, Reiter et al., 2016), antiaging and antiinflammatory properties (for reviews see: Hardeland, 2013; Mauriz et al., 2013), influence on reproductive behavior (for review see, Reiter et al., 2009) as well as sleep (for review see, Dawson and Encel, 1993). However, more recently, it is NAS that has been the subject of much investigation in other research areas.

Melatonin has been identified as a promising avenue of treatment for neurodegenerative disorders such as Parkinson's Disease (for review see, Mack et al., 2016). Comparatively, the potential therapeutic role of NAS has clearly been grossly underestimated in the past and therefore, much investigation in pineal stimulation studies is warranted. This may be considered a limitation of the research pertaining to the therapeutic effects of increasing pineal output discussed in this review, as most of the studies in this area of research have neglected NAS as a significant output of the gland.
The discussed findings in this review illustrate that invasive neuromodulation of the pineal is clearly possible. This is demonstrated through modulation pineal enzymatic activity and indolamine levels. There also exist encouraging results regarding the capability of non-invasive stimulation to produce similar effects. Unfortunately, many of the studies reviewed are lacking in detail regarding experimental protocol and stimulation paradigms. This makes it exceedingly difficult to draw accurate comparisons between the data. Where fair comparisons can be made are generally between data within the same study. It is therefore fortunate that a number of the reviewed pieces of literature were extensive and contain numerous experiments using the same methods. However, caution should be taken when generalising the findings from a handful of studies. Further, there exists little data correlating the electrical activity of pinealocytes with the regulation of melatonin synthesis. This is therefore a limitation of this review as assumptions must be made in order to connect the findings of the evoked cell potential with the indoleamine output and enzymatic activity research. However, the assumptions made have been previously postulated in previous literature and this review makes no attempt to offer novel assumptions in this regard. It is clear that further research is required in order to confirm the findings of previous research, but future studies should take care to conduct experiments using optimal stimulation parameters and be meticulous in detailing experimental variables when reporting results. According to the outcomes of this review, future studies should stimulate the SCG bilaterally at $10 \mathrm{~Hz}$ with a minimum pulse duration of $2 \mathrm{~s}$ and a minimum overall stimulation period of $2 \mathrm{~h}$ during the nighttime in total darkness in order to exert optimal increases in pineal indoleamine levels and enzymatic activity. Further investigation is necessary in this field to uncover whether increases in NAS may result in physiological beneficial outcomes rather than the assumed melatonin, or at least partially.

\section{AUTHOR CONTRIBUTIONS}

YC developed the concept. SL performed the literature review and wrote the draft. SL, AC, and YC analyzed the literature and wrote and edited the main manuscript. SL and YC carried out the diagrams.

\section{ACKNOWLEDGMENTS}

Figure 3 was created with biorender.com. Figure 2 was created by Ella Maru Studio, Inc., United States.

\section{SUPPLEMENTARY MATERIAL}

The Supplementary Material for this article can be found online at: https://www.frontiersin.org/articles/10.3389/fnins. 2020.00264/full\#supplementary-material 


\section{REFERENCES}

Aldhous, M., Franey, C., Wright, J., and Arendt, J. (1985). Plasma concentrations of melatonin in man following oral absorption of different preparations. Br. J. Clin. Pharmacol. 19, 517-521. doi: 10.1111/j.1365-2125.1985.tb02679.x

Al-Hussain, S. M. (2006). The pinealocytes of the human pineal gland: a light and electron microscopic study. Folia Morphol. (Warsz) 65, 181-187.

Bargmann, W. (1943). "Die epiphysis cerebri," in Handbuch der mikroskopischen Anatomie des Menschen, ed. W. von Möllendorff, (Berlin: Springer).

Bazoti, F. M., Tsarbopoulos, A., Markides, K. E., and Bergquist, J. (2005). Study of the non-covalent interaction between amyloid- $\beta$-peptide and melatonin using electrospray ionization mass spectrometry. J. Mass Spectrom. 40, 182-192. doi: 10.1002/jms.738

Berretta, A., Tzeng, Y. C., and Clarkson, A. N. (2014). Post-stroke recovery: the role of activity-dependent release of brain-derived neurotrophic factor. Expert Rev. Neurother. 14, 1335-1344. doi: 10.1586/14737175.2014.969242

Blask, D. E. (2009). Melatonin, sleep disturbance and cancer risk. Sleep Med. Rev. 13, 257-264. doi: 10.1016/j.smrv.2008.07.007

Boeve, B. F., Silber, M. H., and Ferman, T. J. (2003). Melatonin for treatment of REM sleep behavior disorder in neurologic disorders: results in 14 patients. Sleep Med. 4, 281-284. doi: 10.1016/s1389-9457(03)00072-8

Boeve, B. F., Silber, M. H., Ferman, T. J., Lin, S. C., Benarroch, E. E., Schmeichel, A. M., et al. (2013). Clinicopathologic correlations in 172 cases of rapid eye movement sleep behavior disorder with or without a coexisting neurologic disorder. Sleep Med. 14, 754-762. doi: 10.1016/j.sleep.2012.10.015

Bowers, C. W., Baldwin, C., and Zigmond, R. E. (1984a). Sympathetic reinnervation of the pineal-gland after postganglionic nerve lesion does not restore normal pineal function. J. Neurosci. 4, 2010-2015. doi: 10.1523/ jneurosci.04-08-02010.1984

Bowers, C. W., Dahm, L. M., and Zigmond, R. E. (1984b). The number and distribution of sympathetic neurons that innervate the rat pineal-gland. Neuroscience 13, 87-96. doi: 10.1016/0306-4522(84)90261-6

Bowers, C. W., and Zigmond, R. E. (1980). Electrical stimulation of the cervical sympathetic trunks mimics the effects of darkness on the activity of serotonin: $\mathrm{N}$-acetyltransferase in the rat pineal. Brain Res. 185, 435-440. doi: 10.1016/ 0006-8993(80)91082-3

Bowers, C. W., and Zigmond, R. E. (1982). The influence of the frequency and pattern of sympathetic nerve activity on serotonin N-acetyltransferase in the rat pineal gland. J. Physiol. 330, 279-296. doi: 10.1113/jphysiol.1982.sp014341

Brooks, C. M., Ishikawa, T., and Koizumi, K. (1975). Automic system control of the pineal gland and the role of this complex in the integration of body function. Brain Res. 87, 181-190. doi: 10.1016/0006-8993(75)90414-X

Brownstein, M. J., and Heller, A. (1968). Hydroxyindole-O-methyl-transferase activity: effect of sympathetic nerve stimulation. Science 162, 367-368. doi: 10.1126/science.162.3851.367

Buijs, R. M., van Eden, C. G., Goncharuk, V. D., and Kalsbeek, A. (2003). The biological clock tunes the organs of the body: timing by hormones and the autonomic nervous system. J. Endocrinol. 177, 17-26. doi: 10.1677/joe.0. 1770017

Cajochen, C., Krauchi, K., Mori, D., Graw, P., and Wirz-Justice, A. (1997). Melatonin and S-20098 increase REM sleep and wake-up propensity without modifying NREM sleep homeostasis. Am. J. Physiol. 272(4 Pt 2), R1189-R1196. doi: 10.1152/ajpregu.1997.272.4.R1189

Cakmak, Y. O., Akpinar, I. N., Ekinci, G., and Bekiroglu, N. (2008). Pointand frequency-specific response of the testicular artery to abdominal electroacupuncture in humans. Fertil. Steril 90, 1732-1738. doi: 10.1016/j. fertnstert.2007.08.013

Calvo, J., and Boya, J. (1984). Ultrastructure of the pineal gland in the adult rat. J. Anat. 138(Pt 3), 405-409.

Capone, F., Assenza, G., Di Pino, G., Musumeci, G., Ranieri, F., Florio, L., et al. (2015). The effect of transcutaneous vagus nerve stimulation on cortical excitability. J. Neural Trans. 122, 679-685. doi: 10.1007/s00702-0141299-7

Cappuccio, F. P., Taggart, F. M., Kandala, N. B., Currie, A., Peile, E., Stranges, S., et al. (2008). Meta-analysis of short sleep duration and obesity in children and adults. Sleep 31, 619-626. doi: 10.1093/sleep/31.5.619

Carloni, S., Favrais, G., Saliba, E., Albertini, M. C., Chalon, S., Longini, M., et al. (2016). Melatonin modulates neonatal brain inflammation through endoplasmic reticulum stress, autophagy, and miR-34a/silent information regulator 1 pathway. J. Pineal Res. 61, 370-380. doi: 10.1111/jpi.12354

Celnik, P., Hummel, F., Harris-Love, M., Wolk, R., and Cohen, L. G. (2007) Somatosensory stimulation enhances the effects of training functional hand tasks in patients with chronic stroke. Arch. Phys. Med. Rehabil. 88, 1369-1376. doi: 10.1016/j.apmr.2007.08.001

Celnik, P., Paik, N. J., Vandermeeren, Y., Dimyan, M., and Cohen, L. G. (2009). Effects of combined peripheral nerve stimulation and brain polarization on performance of a motor sequence task after chronic stroke. Stroke 40, 17641771. doi: 10.1161/strokeaha.108.540500

Cena, V., Halperin, J. I., Yeandle, S., and Klein, D. C. (1991). Norepinephrine stimulates potassium efflux from pinealocytes: evidence for involvement of biochemical "AND" gate operated by calcium and adenosine 3,5' monophosphate. Endocrinology 128, 559-569. doi: 10.1210/endo-128-1-559

Çetin Altındal, D., James, E. N., Kaplan, D. L., and Gümüşderelioğlu, M. (2019). Melatonin-induced osteogenesis with methanol-annealed silk materials. J. Bioact. Compatible Polym. 34, 291-305. doi: 10.1177/0883911519847489

Chae, H. D., Park, T. J., Lee, Y. K., Lee, T. G., and Kim, K. T. (1999). Rapid and simple measurement of serotonin $\mathrm{N}$-acetyltransferase activity by liquid biphasic diffusion assay. Neurochem. Int. 35, 447-451. doi: 10.1016/s01970186(99)00086-8

Chan, Y. S., Cheung, Y. M., and Pang, S. F. (1989). Elevation of pineal melatonin secretion by electrical-stimulation of the cervical sympathetic trunk in rabbits. Neurosci. Lett. 105, 107-112. doi: 10.1016/0304-3940(89)90020-7

Chao, D. M., Chen, G., and Cheng, J. S. (2001). Melatonin might be one possible medium of electroacupuncture anti-seizures. Acupunct. Electrother. Res. 26, 39-48. doi: 10.3727/036012901816356027

Chattoraj, A., Liu, T., Zhang, L. S., Huang, Z., and Borjigin, J. (2009). Melatonin formation in mammals: in vivo perspectives. Rev. Endocr. Metab. Disord. 10, 237-243. doi: 10.1007/s11154-009-9125-5

Chern, C.-M., Liao, J.-F., Wang, Y.-H., and Shen, Y.-C. (2012). Melatonin ameliorates neural function by promoting endogenous neurogenesis through the MT2 melatonin receptor in ischemic-stroke mice. Free Radic. Biol. Med. 52, 1634-1647. doi: 10.1016/j.freeradbiomed.2012.01.030

Cheung, V., Yuen, V. M., Wong, G. T. C., and Choi, S. W. (2018). The effect of sleep deprivation and disruption on DNA damage and health of doctors. Anaesthesia 74, 434-440. doi: 10.1111/anae.14533

Crupi, R., Mazzon, E., Marino, A., La Spada, G., Bramanti, P., Spina, E., et al. (2011). Melatonin's stimulatory effect on adult hippocampal neurogenesis in mice persists after ovariectomy. J. Pineal Res. 51, 353-360. doi: 10.1111/j.1600079X.2011.00897.x

Curtis, M. A., Kam, M., Nannmark, U., Anderson, M. F., Axell, M. Z., Wikkelso, C., et al. (2007). Human neuroblasts migrate to the olfactory bulb via a lateral ventricular extension. Science 315, 1243-1249. doi: 10.1126/science.1136281

Dauchy, R. T., Wren, M. A., Dauchy, E. M., Hoffman, A. E., Hanifin, J. P., Warfield B., et al. (2015). The influence of red light exposure at night on circadian metabolism and physiology in sprague-dawley rats. J. Am. Assoc. Labor. Anim. Sci. 54, 40-50.

Dawson, D., and Encel, N. (1993). Melatonin and sleep in humans. J. Pineal Res. 15, 1-12. doi: 10.1111/j.1600-079X.1993.tb00503.x

De Ridder, D., and Vanneste, S. (2012). EEG Driven tDCS versus bifrontal tDCS for tinnitus. Front. Psychiatry 3:84. doi: 10.3389/fpsyt.2012.00084

Di Meco, A., Joshi, Y. B., and Pratico, D. (2014). Sleep deprivation impairs memory, tau metabolism, and synaptic integrity of a mouse model of Alzheimer's disease with plaques and tangles. Neurobiol. Aging 35, 1813-1820. doi: 10.1016/j. neurobiolaging.2014.02.011

Dinges, D. F., Douglas, S. D., Hamarman, S., Zaugg, L., and Kapoor, S. (1995). Sleep deprivation and human immune function. Adv. Neuroimmunol. 5, 97-110. doi: 10.1016/0960-5428(95)00002-J

Eccles, R. M. (1955). Intracellular potentials recorded from a mammalian sympathetic ganglion. J. Physiol. 130, 572-584. doi: 10.1113/jphysiol.1955. sp005428

Eriksson, P. S., Perfilieva, E., Bjork-Eriksson, T., Alborn, A. M., Nordborg, C. Peterson, D. A., et al. (1998). Neurogenesis in the adult human hippocampus. Nat. Med. 4, 1313-1317. doi: 10.1038/3305

Faber, M., Vanneste, S., Fregni, F., and De Ridder, D. (2012). Top down prefrontal affective modulation of tinnitus with multiple sessions of tDCS of dorsolateral prefrontal cortex. Brain Stimul. 5, 492-498. doi: 10.1016/j.brs.2011.09.003 
Fernandes, P. A., Cecon, E., Markus, R. P., and Ferreira, Z. S. (2006). Effect of TNF-alpha on the melatonin synthetic pathway in the rat pineal gland: basis for a 'feedback' of the immune response on circadian timing. J. Pineal Res. 41, 344-350. doi: 10.1111/j.1600-079X.2006.00373.x

Fink-Jensen, A., and Møller, M. (1990). Direct projections from the anterior and tuberal regions of the lateral hypothalamus to the rostral part of the pineal complex of the rat. An anterograde neuron-tracing study by usingPhaseolus vulgaris leucoagglutinin. Brain Res. 522, 337-341. doi: 10.1016/0006-8993(90) 91480-5

Frank, E., Schecklmann, M., Landgrebe, M., Burger, J., Kreuzer, P., Poeppl, T. B., et al. (2012). Treatment of chronic tinnitus with repeated sessions of prefrontal transcranial direct current stimulation: outcomes from an open-label pilot study. J. Neurol. 259, 327-333. doi: 10.1007/s00415-011-6189-4

Fregni, F., Boggio, P. S., Lima, M. C., Ferreira, M. J., Wagner, T., Rigonatti, S. P., et al. (2006a). A sham-controlled, phase II trial of transcranial direct current stimulation for the treatment of central pain in traumatic spinal cord injury. Pain 122, 197-209. doi: 10.1016/j.pain.2006.02.023

Fregni, F., Gimenes, R., Valle, A. C., Ferreira, M. J., Rocha, R. R., Natalle, L., et al. (2006b). A randomized, sham-controlled, proof of principle study of transcranial direct current stimulation for the treatment of pain in fibromyalgia. Arthritis Rheum 54, 3988-3998. doi: 10.1002/art.22195

Fregni, F., Boggio, P. S., Mansur, C. G., Wagner, T., Ferreira, M. J. L., Lima, M. C., et al. (2005). Transcranial direct current stimulation of the unaffected hemisphere in stroke patients. Neuroreport 16, 1551-1555. doi: 10.1097/01.wnr. $0000177010.44602 .5 \mathrm{e}$

Freschi, J. E., and Parfitt, A. G. (1986). Intracellular recordings from pineal cells in tissue culture: membrane properties and response to norepinephrine. Brain Res. 368, 366-370. doi: 10.1016/0006-8993(86)90583-4

Garfinkel, D., Laudon, M., Nof, D., and Zisapel, N. (1995). Improvement of sleep quality in elderly people by controlled-release melatonin. Lancet 346, 541-544. doi: 10.1016/s0140-6736(95)91382-3

Gaudet, S., Palkovits, M., and Namboodiri, M. A. (1991). Regional distribution of arylamine and arylalkylamine $\mathrm{N}$-acetyltransferase activities in the rat brain. Brain Res. 539, 355-357. doi: 10.1016/0006-8993(91)91645-h

Gilbey, M. P., Coote, J. H., Fleetwood-Walker, S., and Peterson, D. F. (1982a). The influence of the paraventriculo-spinal pathway, and oxytocin and vasopressin on sympathetic preganglionic neurones. Brain Res. 251, 283-290. doi: 10.1016/ 0006-8993(82)90745-4

Gilbey, M. P., Peterson, D. F., and Coote, J. H. (1982b). Some characteristics of sympathetic preganglionic neurones in the rat. Brain Res. 241, 43-48. doi: 10.1016/0006-8993(82)91226-4

Goto, M., Oshima, I., Tomita, T., and Ebihara, S. (1989). Melatonin content of the pineal gland in different mouse strains. J. Pineal Res. 7, 195-204. doi: 10.1111/j.1600-079x.1989.tb00667.x

Gould, E., Reeves, A. J., Fallah, M., Tanapat, P., Gross, C. G., and Fuchs, E. (1999). Hippocampal neurogenesis in adult Old World primates. Proc. Natl. Acad. Sci. U.S.A. 96, 5263-5267. doi: 10.1073/pnas.96.9.5263

Haimov, I., Laudon, M., Zisapel, N., Souroujon, M., Nof, D., Shlitner, A., et al. (1994). Sleep disorders and melatonin rhythms in elderly people. Br. Med. J. 309, 167-167. doi: 10.1136/bmj.309.6948.167

Hansen, J. (2001). Increased breast cancer risk among women who work predominantly at night. Epidemiology 12, 74-77. doi: 10.1097/00001648200101000-00013

Hardeland, R. (2013). Melatonin and the theories of aging: a critical appraisal of melatonin's role in antiaging mechanisms. J. Pineal Res. 55, 325-356. doi: 10.1111/jpi.12090

Hardy, J. A., and Higgins, G. A. (1992). Alzheimer's disease: the amyloid cascade hypothesis. Science 256, 184-185.

Hassinger, T. D., Atkinson, P. B., Strecker, G. J., Whalen, L. R., Dudek, F. E., Kossel, A. H., et al. (1995). Evidence for glutamate-mediated activation of hippocampal neurons by glial calcium waves. J. Neurobiol. 28, 159-170. doi: 10.1002/neu. 480280204

Heidbuchel, U., and Vollrath, L. (1983). Pineal complex of rats: effects of superficial pinealectomy on the deep pineal. Acta Anat. (Basel) 117, 165-169. doi: 10.1159/ 000145782

Hendrickson, A. E., Wagoner, N., and Cowan, W. M. (1972). Autoradiogrpahic and electron-microscopic study of retino-hypothalamic connections. Z. Zellforschung Mikroskopische Anat. 135:1. doi: 10.1007/bf00307084
Heydorn, W. E., Frazer, A., and Weiss, B. (1981). Electrical stimulation of sympathetic nerves increases the concentration of cyclic AMP in rat pineal gland. Proc. Natl. Acad. Sci. U.S.A. 78, 7176-7179. doi: 10.1073/pnas.78.11.7176

Hoevenaar-Blom, M. P., Spijkerman, A. M., Kromhout, D., van den Berg, J. F., and Verschuren, W. M. (2011). Sleep duration and sleep quality in relation to 12 -year cardiovascular disease incidence: the MORGEN study. Sleep 34, 1487-1492. doi: 10.5665/sleep.1382

Hoshino, M., Kaneko, K., Miyamoto, Y., Yoshimura, K., Suzuki, D., Akaike, T., et al. (2017). 8-Nitro-cGMP promotes bone growth through expansion of growth plate cartilage. Free Radic. Biol. Med. 110, 63-71. doi: 10.1016/j. freeradbiomed.2017.05.022

Hu, C. X., and Li, L. J. (2019). Melatonin plays critical role in mesenchymal stem cell-based regenerative medicine in vitro and in vivo. Stem Cell Res. Ther. 10:11.

Huang, H. T., and Lin, H. S. (1984). Synaptic junctions between the adrenergic axon varicosity and the pinealocyte in the rat. J. Pineal Res. 1, 281-291. doi: 10.1111/j.1600-079X.1984.tb00219.x

Huether, G. (1993). The contribution of extrapineal sites of melatonin synthesis to circulating melatonin levels in higher vertebrates. Experientia 49, 665-670. doi: 10.1007/bf01923948

Hughes, R. J., Sack, R. L., and Lewy, A. J. (1998). The role of melatonin and circadian phase in age-related sleep-maintenance insomnia: assessment in a clinical trial of melatonin replacement. Sleep 21, 52-68.

Hummel, F., Celnik, P., Giraux, P., Floel, A., Wu, W. H., Gerloff, C., et al. (2005). Effects of non-invasive cortical stimulation on skilled motor function in chronic stroke. Brain 128, 490-499. doi: 10.1093/brain/awh369

Hummel, F. C., Voller, B., Celnik, P., Floel, A., Giraux, P., Gerloff, C., et al. (2006). Effects of brain polarization on reaction times and pinch force in chronic stroke. BMC Neurosci. 7:73. doi: 10.1186/1471-2202-7-73

International Neuromodulation Society (2018). Welcome to the International Neuromodulation Society. Available: https://www.neuromodulation.com/ neuromodulation-defined [Accessed 14 November 2018 2018].

Ito, M., Kadekaro, M., and Sokoloff, L. (1988). Local glucose-utilization of the brain and pineal-gland during stimulation of the cervical sympathetic trunk. J. Pineal Res. 5, 51-62. doi: 10.1111/j.1600-079X.1988.tb00768.x

Jang, S. W., Liu, X., Pradoldej, S., Tosini, G., Chang, Q., Iuvone, P. M., et al. (2010). $\mathrm{N}$-acetylserotonin activates TrkB receptor in a circadian rhythm. Proc. Natl. Acad. Sci. U.S.A. 107, 3876-3881. doi: 10.1073/pnas.0912531107

Joos, K., De Ridder, D., Van de Heyning, P., and Vanneste, S. (2014). Polarity specific suppression effects of transcranial direct current stimulation for tinnitus. Neural Plast. 2014:930860. doi: 10.1155/2014/930860

Kalsbeek, A., Garidou, M. L., Palm, I. F., van der Vliet, J., Simonneaux, V., Pevet, P., et al. (2000). Melatonin sees the light: blocking GABA-ergic transmission in the paraventricular nucleus induces daytime secretion of melatonin. European Journal of Neuroscience 12, 3146-3154. doi: 10.1046/j.1460-9568.2000.00202.x

Kannan, H., Hayashida, Y., and Yamashita, H. (1989). Increase in sympathetic outflow by paraventricular nucleus stimulation in awake rats. Am. J. Physiol. 256(6 Pt 2), R1325-R1330. doi: 10.1152/ajpregu.1989.256.6.R1325

Kannan, H., Niijima, A., and Yamashita, H. (1987). Inhibition of renal sympathetic nerve activity by electrical stimulation of the hypothalamic paraventricular nucleus in anesthetized rats. J. Auton. Nerv. Syst. 21, 83-86. doi: 10.1016/01651838(87)90094-4

Kappers, J. A. (1960a). Innervation of the epiphysis cerebri in the albino rat. Anatom. Rec. 136, 220-221.

Kappers, J. A. (1960b). The development, topographical relations and innervation of the epiphysis cerebri in the albino rat. Z. Zellforschung Mikroskop. Anat. 52, 163-215. doi: $10.1007 / \mathrm{bf} 00338980$

Kappers, J. A. (1965). Survey of the innervation of the epiphysis cerebri and the accessory pineal organs of vertebrates. Prog. Brain Res. 10, 87-153. doi: 10.1016/ s0079-6123(08)63448-2

Kayumov, L., Spence, W., Lowe, A., Hawa, R., Koszorowska, M., Jain, U., et al. (2003). Acupuncture reduces depressive and insomnia symptoms, and increases nocturnal melatonin secretion. Sleep 26, A291-A292.

Kenny, G. C. (1967). Innervation of the mammalian pineal body. Anat. Rec. 157:269.

Khedr, E. M., Ahmed, M. A., Fathy, N., and Rothwell, J. C. (2005). Therapeutic trial of repetitive transcranial magnetic stimulation after acute ischemic stroke. Neurology 65, 466-468. doi: 10.1212/01.wnl.0000173067.84247.36 
Kim, M. J., Kim, H. K., Kim, B. S., and Yim, S. V. (2004). Melatonin increases cell proliferation in the dentate gyrus of maternally separated rats. J. Pineal Res. 37, 193-197. doi: 10.1111/j.1600-079X.2004.00157.x

Kim, Y. H., You, S. H., Ko, M. H., Park, J. W., Lee, K. H., Jang, S. H., et al. (2006). Repetitive transcranial magnetic stimulation-induced corticomotor excitability and associated motor skill acquisition in chronic stroke. Stroke 37, 1471-1476. doi: 10.1161/01.Str.0000221233.55497.51

Klein, D. C., and Weller, J. L. (1972). Rapid light-induced decrease in pineal serotonin $\mathrm{N}$-acetyltransferase activity. Science 177, 532-533. doi: 10.1126/ science.177.4048.532

Korf, H. W., and Wagner, U. (1980). Evidence for a nervous connection between the brain and the pineal organ in the guinea-pig. Cell Tissue Res. 209, 505-510.

Krause, A. J., Prather, A. A., Wager, T. D., Lindquist, M. A., and Walker, M. P. (2019). The pain of sleep loss: a brain characterization in humans. J. Neurosci. 39, 2291. doi: 10.1523/JNEUROSCI.2408-18.2018

Kreuzer, P. M., Landgrebe, M., Husser, O., Resch, M., Schecklmann, M., and Geisreiter, F. (2012). Transcutaneous vagus nerve stimulation: retrospective assessment of cardiac safety in a pilot study. Front. Psychiatry 3:70. doi: 10.3389/ fpsyt.2012.00070

Kreuzer, P. M., Landgrebe, M., Resch, M., Husser, O., Schecklmann, M., Geisreiter, F., et al. (2014). Feasibility, safety and efficacy of transcutaneous vagus nerve stimulation in chronic tinnitus: an open pilot study. Brain Stimul. 7, 740-747. doi: 10.1016/j.brs.2014.05.003

Kubo, T., Ozasa, K., Mikami, K., Wakai, K., Fujino, Y., Watanabe, Y., et al. (2006). Prospective cohort study of the risk of prostate cancer among rotating-shift workers: findings from the Japan collaborative cohort study. Am. J. Epidemiol. 164, 549-555. doi: 10.1093/aje/kwj232

Kumar, A., and Chanana, P. (2014). Sleep reduction: a link to other neurobiological diseases. Sleep Biol. Rhythms 12, 150-161. doi: 10.1111/sbr.12066

Kunz, D., and Bes, F. (1999). Melatonin as a therapy in REM sleep behavior disorder patients: an open-labeled pilot study on the possible influence of melatonin on REM-sleep regulation. Mov. Disord. 14, 507-511. doi: 10.1002/ 1531-8257(199905)14:3<507::aid-mds1021>3.0.co;2-8

Kunz, D., and Mahlberg, R. (2010). A two-part, double-blind, placebo-controlled trial of exogenous melatonin in REM sleep behaviour disorder. J. Sleep Res. 19, 591-596. doi: 10.1111/j.1365-2869.2010.00848.x

Kunz, D., Mahlberg, R., Muller, C., Tilmann, A., and Bes, F. (2004). Melatonin in patients with reduced REM sleep duration: two randomized controlled trials. J. Clin. Endocrinol. Metab. 89, 128-134. doi: 10.1210/jc.2002-021057

Larsen, P. J., Møller, M., and Mikkelsen, J. D. (1991). Efferent projections from the periventricular and medial parvicellular subnuclei of the hypothalamic paraventricular nucleus to circumventricular organs of the rat: a Phaseolus vulgaris-leucoagglutinin (PHA-L) tracing study. J. Compar. Neurol. 306, 462 479. doi: 10.1002/cne.903060310

le Gros Clark, W. E. (1940). The nervous and vascular relations of the pineal gland. J. Anat. 74(Pt 4), 471-492.

Leander, P., Vrang, N., and Moller, M. (1998). Neuronal projections from the mesencephalic raphe nuclear complex to the suprachiasmatic nucleus and the deep pineal gland of the golden hamster (Mesocricetus auratus). J. Comp. Neurol. 399, 73-93. doi: 10.1002/(sici)1096-9861(19980914)399:1<73:: aid-cne6 $>3.0 . \operatorname{co} ; 2-7$

Lefaucheur, J. P., Drouot, X., Keravel, Y., and Nguyen, J. P. (2001). Pain relief induced by repetitive transcranial magnetic stimulation of precentral cortex. Neuroreport 12, 2963-2965. doi: 10.1097/00001756-200109170-00041

Lemoine, P., and Zisapel, N. (2012). Prolonged-release formulation of melatonin (Circadin) for the treatment of insomnia. Expert Opin. Pharmacother. 13, 895-905. doi: 10.1517/14656566.2012.667076

Leone, R. M., and Silman, R. E. (1984). Melatonin can be differentially metabolized in the rat to produce $\mathrm{N}$-acetylserotonin in addition to 6-hydroxy-melatonin. Endocrinology 114, 1825-1832. doi: 10.1210/endo-114-5-1825

Lewy, A. J., and Newsome, D. A. (1983). Different types of melatonin circadian secretory rhythms in some blind subjects. J. Clin. Endocrinol. Metab. 56, 1103-1107. doi: 10.1210/jcem-56-6-1103

Lewy, A. J., Tetsuo, M., Markey, S. P., Goodwin, F. K., and Kopin, I. J. (1980). Pinealectomy abolishes plasma melatonin in the rat. J. Clin. Endocrinol. Metab. 50, 204-205. doi: 10.1210/jcem-50-1-204

Li, S., Zhai, X., Rong, P., McCabe, M. F., Zhao, J., Ben, H., et al. (2014). Transcutaneous auricular vagus nerve stimulation triggers melatonin secretion and is antidepressive in zucker diabetic fatty rats. PLOS ONE 9:e111100. doi: 10.1371/journal.pone.0111100

Lin, C., Chao, H., Li, Z., Xu, X., Liu, Y., Hou, L., et al. (2016). Melatonin attenuates traumatic brain injury-induced inflammation: a possible role for mitophagy. J. Pineal Res. 61, 177-186. doi: 10.1111/jpi.12337

Lingappa, J. R., and Zigmond, R. E. (1987). A histochemical study of the adrenergic innervation of the rat pineal gland: evidence for overlap of the innervation from the two superior cervical ganglia and for sprouting following unilateral denervation. Neuroscience 21, 893-902. doi: 10.1016/0306-4522(87)90045-5

Lingappa, J. R., and Zigmond, R. E. (2013). Limited recovery of pineal function after regeneration of preganglionic sympathetic axons: evidence for loss of ganglionic synaptic specificity. J. Neurosci. 33, 4867-4874. doi: 10.1523/ jneurosci.3829-12.2013

Lingford- Hughes, A., and Kalk, N. (2012). Core Psychiatry, 3rd Edn. London: Elsevier.

Liu, L. D., Prescott, I. A., Dostrovsky, J. O., Hodaie, M., Lozano, A. M., and Hutchison, W. D. (2012). Frequency-dependent effects of electrical stimulation in the globus pallidus of dystonia patients. J. Neurophysiol. 108, 5-17. doi: 10.1152/jn.00527.2011

Liu, R. Y., Zhou, J. N., van Heerikhuize, J., Hofman, M. A., and Swaab, D. F. (1999). Decreased melatonin levels in postmortem cerebrospinal fluid in relation to aging, Alzheimer's disease, and apolipoprotein E-epsilon 4/4 genotype. J. Clin. Endocrinol. Metab. 84, 323-327. doi: 10.1210/jc.84. 1.323

Liu, T., and Borjigin, J. (2005). N-acetyltransferase is not the rate-limiting enzyme of melatonin synthesis at night. J. Pineal Res. 39, 91-96. doi: 10.1111/j.1600079X.2005.00223.x

Lledo, P. M., Alonso, M., and Grubb, M. S. (2006). Adult neurogenesis and functional plasticity in neuronal circuits. Nat. Rev. Neurosci. 7, 179-193. doi: 10.1038/nrn1867

Luo, C., Yang, Q., Liu, Y., Zhou, S., Jiang, J., Reiter, R. J., et al. (2019). The multiple protective roles and molecular mechanisms of melatonin and its precursor $\mathrm{N}$-acetylserotonin in targeting brain injury and liver damage and in maintaining bone health. Free Radic. Biol. Med. 130, 215-233. doi: 10.1016/j. freeradbiomed.2018.10.402

Ma, D. K., Kim, W. R., Ming, G. L., and Song, H. (2009). Activity-dependent extrinsic regulation of adult olfactory bulb and hippocampal neurogenesis. Ann. N. Y. Acad. Sci. 1170, 664-673. doi: 10.1111/j.1749-6632.2009.04373.x

Mack, J. M., Schamne, M. G., Sampaio, T. B., Pértile, R. A. N., Fernandes, P. A. C. M., Markus, R. P., et al. (2016). Melatoninergic system in Parkinson's disease: from neuroprotection to the management of motor and nonmotor symptoms. Oxidat. Med. Cell. Longevity 2016, 3472032-3472032. doi: 10.1155/ 2016/3472032

Manda, K., and Reiter, R. J. (2010). Melatonin maintains adult hippocampal neurogenesis and cognitive functions after irradiation. Prog. Neurobiol. 90, 60-68. doi: 10.1016/j.pneurobio.2009.10.019

Mansur, C. G., Fregni, F., Boggio, P. S., Riberto, M., Gallucci-Neto, J., Santos, C. M., et al. (2005). A sham stimulation-controlled trial of rTMS of the unaffected hemisphere in stroke patients. Neurology 64, 1802-1804. doi: 10.1212/01.Wnl. 0000161839.38079 .92

Matsushima, S., Sakai, Y., Hira, Y., Oomori, Y., and Daikoku, S. (1994). Immunohistochemical studies on sympathetic and non-sympathetic nerve fibers and neuronal cell bodies in the pineal gland of cotton rats, Sigmodon hispidus. Arch. Histol. Cytol. 57, 47-58. doi: 10.1679/aohc. 57.47

Matsuura, T., Kumamoto, K., and Ebara, S. (1994). Nerve-fibers originating from the brain in the rat pineal complex. J. Electron. Microsc. 43, 255-263.

Mauriz, J. L., Collado, P. S., Veneroso, C., Reiter, R. J., and Gonzalez-Gallego, J. (2013). A review of the molecular aspects of melatonin's anti-inflammatory actions: recent insights and new perspectives. J. Pineal Res. 54, 1-14. doi: 10. 1111/j.1600-079X.2012.01014.x

Mays, J. C., Kelly, M. C., Coon, S. L., Holtzclaw, L., Rath, M. F., Kelley, M. W., et al. (2018). Single-cell RNA sequencing of the mammalian pineal gland identifies two pinealocyte subtypes and cell type-specific daily patterns of gene expression. PLoS ONE 13:e0205883. doi: 10.1371/journal.pone.0205883

McAlpine, C. S., Kiss, M. G., Rattik, S., He, S., Vassalli, A., Valet, C., et al. (2019). Sleep modulates haematopoiesis and protects against atherosclerosis. Nature 566, 383-387. doi: 10.1038/s41586-019-0948-2 
McIntyre, I. M., and Oxenkrug, G. F. (1984). Electroconvulsive shock: effect on pineal and hypothalamic indoles. J. Pineal Res. 1, 273-279. doi: 10.1111/j.1600079x.1984.tb00218.x

Mikkelsen, J. D., and Moller, M. (1990). A direct neural projection from the intergeniculate leaflet of the lateral geniculate nucleus to the deep pineal gland of the rat, demonstrated with Phaseolus vulgaris leucoagglutinin. Brain Res. 520, 342-346. doi: 10.1016/0006-8993(90)91727-x

Minchin, M. C., and Iversen, L. L. (1974). Release of (3H)gamma-aminobutyric acid from glial cells in rat dorsal root ganglia. J. Neurochem. 23, 533-540. doi: $10.1111 / j .1471-4159.1974 . t b 06056 . x$

Ming, G. L., and Song, H. (2005). Adult neurogenesis in the mammalian central nervous system. Annu. Rev. Neurosci. 28, 223-250. doi: 10.1146/annurev.neuro. 28.051804.101459

Mirick, D. K., Stevens, R. G., and Davis, S. (2001). Night shift work, light at night, and risk of breast cancer. J. Natl. Cancer Inst. 93, 1557-1562. doi: 10.1093/jnci/ 93.20.1557

Moller, M. (1978). Presence of a pineal nerve (nervus pinealis) in the human fetus: a light and electron microscopical study of the innervation of the pineal gland. Brain Res. 154, 1-12. doi: 10.1016/0006-8993(78)91046-6

Moller, M., and Hay-Schmidt, A. (1998). Direct neuronal projection from the dorsal raphe nucleus to the pineal complex of the rat: a Phaseolus vulgarisleucoagglutinin in vivo neuronal tracing study. J. Pineal Res. 25, 19-23. doi: 10.1111/j.1600-079x.1998.tb00381.x

Moller, M., and Korf, H. W. (1983). Central innervation of the pineal organ of the Mongolian gerbil. A histochemical and lesion study. Cell Tissue Res. 230, 259-272.

Moller, M., Phansuwan-Pujito, P., Govitrapong, P., and Schmidt, P. (1993). Indications for a central innervation of the bovine pineal gland with substance P-immunoreactive nerve fibers. Brain Res. 611, 347-351. doi: 10.1016/00068993(93)90525-r

Moore, R. Y. (1973). Retinohypothalamic projection in mammals - comparative study. Brain Res. 49, 403-409. doi: 10.1016/0006-8993(73)90431-9

Moore, R. Y., and Lenn, N. J. (1972). Retinohypothalamic projection in rat. J. Compar. Neurol. 146, 1-14. doi: 10.1002/cne.901460102

Mukda, S., Panmanee, J., Boontem, P., and Govitrapong, P. (2016). Melatonin administration reverses the alteration of amyloid precursor protein-cleaving secretases expression in aged mouse hippocampus. Neurosci. Lett. 621, 39-46. doi: 10.1016/j.neulet.2016.04.013

Munch, I. C., Moller, M., Larsen, P. J., and Vrang, N. (2002). Light-induced c-fos expression in suprachiasmatic nuclei neurons targeting the paraventricular nucleus of the hamster hypothalamus: phase dependence and immunochemical identification. J. Compar. Neurol. 442, 48-62. doi: 10.1002/cne.1421

Munoz-Hoyos, A., Sanchez-Forte, M., Molina-Carballo, A., Escames, G., MartinMedina, E., Reiter, R. J., et al. (1998). Melatonin's role as an anticonvulsant and neuronal protector: experimental and clinical evidence. J. Child Neurol. 13, 501-509. doi: 10.1177/088307389801301007

Murali, R., Thanikaivelan, P., and Cheirmadurai, K. (2016). Melatonin in functionalized biomimetic constructs promotes rapid tissue regeneration in Wistar albino rats. J. Mater. Chem. B 4, 5850-5862. doi: 10.1039/c6tb01221c

Netter, F. H. (1999). The Netter Collection of Medical Illustrations: Nervous System Anatomy and Physiology. Amsterdam: Elsevier.

Neuwelt, E. A., and Lewy, A. J. (1983). Disappearance of plasma melatonin after removal of a neoplastic pineal gland. N. Engl. J. Med. 308, 1132-1135. doi: 10.1056/nejm198305123081905

Ngai, A. C., Jolley, M. A., D’Ambrosio, R., Meno, J. R., and Winn, H. R. (1999). Frequency-dependent changes in cerebral blood flow and evoked potentials during somatosensory stimulation in the rat. Brain Res. 837, 221-228. doi: 10.1016/s0006-8993(99)01649-2

Nosjean, O., Ferro, M., Coge, F., Beauverger, P., Henlin, J. M., Lefoulon, F., et al. (2000). Identification of the melatonin-binding site MT3 as the quinone reductase 2. J. Biol. Chem. 275, 31311-31317. doi: 10.1074/jbc.M005141200

Nowak, J. Z., Przybysz, M., and Zurawska, E. (1988). The melatonin generating system in the rat retina and pineal gland: effect of single and repeated electroconvulsive shock (ECS). Pol. J. Pharmacol. Pharm. 40, 573-584.

Olcese, J., Reuss, S., and Steinlechner, S. (1987). Electrical stimulation of the hypothalamic nucleus paraventricularis mimics the effects of light on pineal melatonin synthesis. Life Sci. 40, 455-459. doi: 10.1016/0024-3205(87) 90110-x
Ooms, S., Overeem, S., Besse, K., Rikkert, M. O., Verbeek, M., and Claassen, J. A. (2014). Effect of 1 night of total sleep deprivation on cerebrospinal fluid betaamyloid 42 in healthy middle-aged men: a randomized clinical trial. JAMA Neurol. 71, 971-977. doi: 10.1001/jamaneurol.2014.1173

Oxenkrug, G., and Ratner, R. (2012). N-acetylserotonin and aging-associated cognitive impairment and depression. Aging Dis. 3, 330-338.

Oxenkrug, G. F., Requintina, P. J., McIntyre, I. M., and Davis, R. (1991). "Stimulation of rat pineal melatonin synthesis by a single electroconvulsive shock - chronobiological effect of antidepressant therapy," in 5Hydroxytryptamine in Psychiatry: a Spectrum of Ideas, eds M. Sandler, A. Coppen, and S. Harnett, (Oxford: Oxford University Press), 110-115. doi: 10.1093/acprof:oso/9780192620118.003.0009

Panmanee, J., Nopparat, C., Chavanich, N., Shukla, M., Mukda, S., Song, W., et al. (2015). Melatonin regulates the transcription of $\beta A P P$-cleaving secretases mediated through melatonin receptors in human neuroblastoma SH-SY5Y cells. J. Pineal Res. 59, 308-320. doi: 10.1111/jpi.12260

Pappolla, M., Bozner, P., Soto, C., Shao, H., Robakis, N. K., Zagorski, M., et al. (1998). Inhibition of Alzheimer beta-fibrillogenesis by melatonin. J. Biol. Chem. 273, 7185-7188. doi: 10.1074/jbc.273.13.7185

Parfitt, A., Weller, J. L., Klein, D. C., Sakai, K. K., and Marks, B. H. (1975). Blockade by ouabain or elevated potassium ion concentration of the adrenergic and adenosine cyclic 3',5'-monophosphate-induced stimulation of pineal serotonin $\mathrm{N}$-acetyltransferase activity. Mol. Pharmacol. 11, 241-255.

Parpura, V., Basarsky, T. A., Liu, F., Jeftinija, K., Jeftinija, S., and Haydon, P. G. (1994). Glutamate-mediated astrocyte-neuron signalling. Nature 369, 744-747. doi: $10.1038 / 369744 \mathrm{a} 0$

Patel, S., and Demaine, C. (1990). Effects of stimulation of the superior cervical Ganglia and local application of noradrenaline on electrical activity of the Syrian hamster pineal gland. J. Neuroendocrinol. 2, 493-499. doi: 10.1111/j. 1365-2826.1990.tb00438.x

Paul, S. M., Hsu, L. L., and Mandell, A. J. (1974). Extrapineal N-acetyltransferase activity in rat brain. Life Sci. 15, 2135-2143. doi: 10.1016/0024-3205(74)90030-7

Pazo, J. H. (1981). Electrophysiological study of evoked electrical activity in the pineal gland. J. Neural Transm. 52, 137-148. doi: 10.1007/bf01253105

Pazo, J. H., and Gonzalez, M. (1991). Effects of central and peripheral inputs on single pineal cell-activity in the rat. Neuroscience 43, 231-235. doi: 10.1016/ 0306-4522(91)90430-v

Peng, C.-X., Hu, J., Liu, D., Hong, X.-P., Wu, Y.-Y., Zhu, L.-Q., et al. (2013). Disease-modified glycogen synthase kinase- $3 \beta$ intervention by melatonin arrests the pathology and memory deficits in an Alzheimer's animal model. Neurobiol. Aging 34, 1555-1563. doi: 10.1016/j.neurobiolaging.2012.12.010

Pfeffer, M., Korf, H.-W., and Wicht, H. (2018). Synchronizing effects of melatonin on diurnal and circadian rhythms. Gen. Compar. Endocrinol. 258, 215-221. doi: 10.1016/j.ygcen.2017.05.013

Psarakis, S., Pulido, O. M., Brown, G. M., Grota, L. J., and Smith, G. K. (1982). Identification and quantification of $\mathrm{n}$-acetylserotonin (NAS) in the developing hippocampus of the rat. Prog. Neuropsychopharmacol. Biol. Psychiatry 6, 439442. doi: 10.1016/s0278-5846(82)80124-3

Qian, Y., Han, Q., Zhao, X., Song, J., Cheng, Y., Fang, Z., et al. (2018). 3D melatonin nerve scaffold reduces oxidative stress and inflammation and increases autophagy in peripheral nerve regeneration. J. Pineal Res. 65:e12516. doi: 10.1111/jpi.12516

Quay, W. B. (1973). Retrograde perfusions of the pineal region and the question of pineal vascular routes to brain and choroid plexuses. Am. J. Anat. 137, 387-401. doi: 10.1002/aja.1001370403

Ramirez-Rodriguez, G., Klempin, F., Babu, H., Benitez-King, G., and Kempermann, G. (2009). Melatonin modulates cell survival of new neurons in the hippocampus of adult mice. Neuropsychopharmacology 34, 2180-2191. doi: $10.1038 /$ npp.2009.46

Ramirez-Rodriguez, G., Ortiz-Lopez, L., Dominguez-Alonso, A., Benitez-King, G. A., and Kempermann, G. (2011). Chronic treatment with melatonin stimulates dendrite maturation and complexity in adult hippocampal neurogenesis of mice. J. Pineal Res. 50, 29-37. doi: 10.1111/j.1600-079X.2010. 00802.x

Rath, M. F., Coon, S. L., Amaral, F. G., Weller, J. L., Moller, M., and Klein, D. C. (2016). Melatonin synthesis: acetylserotonin o-methyltransferase (ASMT) is strongly expressed in a subpopulation of pinealocytes in the male rat pineal gland. Endocrinology 157, 2028-2040. doi: 10.1210/en.2015-1888 
Reiter, R. J. (1990). Biological Rhythms, Mood Disorders, Light Therapy, and the Pineal Gland. Washington, DC: American Psychiatric Press.

Reiter, R. J., Mayo, J. C., Tan, D. X., Sainz, R. M., Alatorre-Jimenez, M., and Qin, L. L. (2016). Melatonin as an antioxidant: under promises but over delivers. J. Pineal Res. 61, 253-278. doi: 10.1111/jpi.12360

Reiter, R. J., Tan, D. X., Manchester, L. C., Paredes, S. D., Mayo, J. C., and Sainz, R. M. (2009). Melatonin and reproduction revisited. Biol. Reprod. 81, 445-456. doi: 10.1095/biolreprod.108.075655

Reiter, R. J., Tan, D. X., Poeggeler, B., Menendez-Pelaez, A., Chen, L. D., and Saarela, S. (1994). Melatonin as a free radical scavenger: implications for aging and age-related diseasesa. Ann. N. Y. Acad. Sci. 719, 1-12. doi: 10.1111/j.17496632.1994.tb56817.x

Reiter, R. J., Tan, D. X., Rosales-Corral, S., and Manchester, L. C. (2013). The universal nature, unequal distribution and antioxidant functions of melatonin and its derivatives. Mini Rev. Med. Chem. 13, 373-384. doi: 10.2174/ 1389557511313030006

Rennie, K., De Butte, M., and Pappas, B. A. (2009). Melatonin promotes neurogenesis in dentate gyrus in the pinealectomized rat. J. Pineal Res. 47, 313-317. doi: 10.1111/j.1600-079X.2009.00716.x

Reuss, S. (1986). Effects of chemical and surgical ganglionectomy on electrical activity of the pineal gland of male rats. J. Pineal Res. 3, 87-94. doi: 10.1111/ j.1600-079x.1986.tb00729.x

Reuss, S. (1999). Trigeminal innervation of the mammalian pineal gland. Microsc. Res. Tech. 46, 305-309. doi: 10.1002/(sici)1097-0029(19990815/01)46:4/5<305:: aid-jemt7>3.0.co;2-\#

Reuss, S., and Moller, M. (1986). Direct projections to the rat pineal gland via the stria medullaris thalami. An anterograde tracing study by use of horseradish peroxidase. Cell Tissue Res. 244, 691-694.

Reuss, S., Concemius, W., Stehle, J., Seidel, A., Schröder, H., and Vollrath, L. (1989). Effects of electrical stimulation of the superior cervical ganglia on the number of "synaptic" ribbons and the activity of melatonin-forming enzymes in the rat pineal gland. Anat. Embryol. 179, 341-345. doi: 10.1007/bf00305060

Reuss, S., Olcer, S., and Vollrath, L. (1985a). Electrical stimulation of the hypothalamic paraventricular nuclei inhibits pineal melatonin synthesis in male rats. Neuroendocrinology 41, 192-196. doi: 10.1159/000124177

Reuss, S., Semm, P., and Vollrath, L. (1985b). Changes in the electrical activity of the rat pineal gland following stimulation of the cervical sympathetic ganglia. J. Auton. Nerv. Syst. 12, 281-288. doi: 10.1016/0165-1838(85)90043-8

Reuss, S., Semm, P., and Vollrath, L. (1984). Electrophysiological investigations on the central innervation of the rat and guinea-pig pineal gland. J. Neural Trans. 60, 31-43. doi: 10.1007/bf01254763

Reyes-Vazquez, C., Prieto-Gomez, B., Aldes, L. D., and Dafny, N. (1986). Rat pineal exhibits two electrophysiological patterns of response to microiontophoretic norepinephrine application. J. Pineal Res. 3, 213-222. doi: 10.1111/j.1600-079x. 1986.tb00744.x

Rodriguez, C., Mayo, J. C., Sainz, R. M., Antolín, I., Herrera, F., Martín, V., et al. (2004). Regulation of antioxidant enzymes: a significant role for melatonin. J. Pineal Res. 36, 1-9. doi: 10.1046/j.1600-079X.2003.00092.x

Rodriguez-Perez, A. (1962). Contribución al conocimiento de la inervación de las glándulas endocrinas. IV. Primeros resultados experimentales en torno a la inervación de la epífisis. Trab. Inst. Cajal Invest. Biol. 54, 1-8.

Romijn, H. J. (1975). Structure and innervation of the pineal gland of the rabbit, oryctolagus cuniculus. Cell Tissue Res. 157, 25-51. doi: 10.1007/bf00223229

Ronnekleiv, O. K., Kelly, M. J., and Wuttke, W. (1980). Single unit recordings in the rat pineal gland: evidence for habenulo-pineal neural connections. Exp. Brain Res. 39, 187-192.

Rosenstein, R. E., Chuluyan, H. E., Pereyra, E. N., and Cardinali, D. P. (1989). Release and effect of gamma-aminobutyric acid (GABA) on rat pineal melatonin production in vitro. Cell Mol. Neurobiol. 9, 207-219. doi: 10.1007/ bf00713029

Rossini, P. M., Burke, D., Chen, R., Cohen, L. G., Daskalakis, Z., Di Iorio, R., et al. (2015). Non-invasive electrical and magnetic stimulation of the brain, spinal cord, roots and peripheral nerves: basic principles and procedures for routine clinical and research application. An updated report from an IFCN Committee. Clin. Neurophysiol. 126, 1071-1107. doi: 10.1016/j.clinph.2015.02.001

Rothman, S. M., Herdener, N., Frankola, K. A., Mughal, M. R., and Mattson, M. P. (2013). Chronic mild sleep restriction accentuates contextual memory impairments, and accumulations of cortical $\mathrm{A} \beta$ and pTau in a mouse model of Alzheimer's disease. Brain Res. 1529, 200-208. doi: 10.1016/j.brainres.2013. 07.010

Sakai, K. K., and Marks, B. H. (1972). Adrenergic effects on pineal cell membrane potential. Life Sci. I 11, 285-291. doi: 10.1016/0024-3205(72)90231-7

Savaskan, E., Jockers, R., Ayoub, M., Angeloni, D., Fraschini, F., Flammer, J., et al. (2007). The MT2 melatonin receptor subtype is present in human retina and decreases in Alzheimer's disease. Curr. Alzheimer Res. 4, 47-51. doi: 10.2174/ 156720507779939823

Schaefer, C., Kunz, D., and Bes, F. (2017). Melatonin effects in REM sleep behavior disorder associated with obstructive sleep apnea syndrome: a case series. Curr. Alzheimer Res. 14, 1084-1089. doi: 10.2174/1567205014666170523094938

Schapiro, S., and Salas, M. (1971). Effects of age, light and sympathetic innervation on electrical activity of rat pineal gland. Brain Res. 28, 47-55. doi: 10.1016/00068993(71)90523-3

Schenda, J., and Vollrath, L. (1997). Nitric oxide inhibits electrically active units in the rat pineal gland. J. Neural Transm. (Vienna) 104, 53-58. doi: 10.1007/ bf01271293

Schenda, J., and Vollrath, L. (1999). An intrinsic neuronal-like network in the rat pineal gland. Brain Res. 823, 231-233. doi: 10.1016/s0006-8993(99)01199-3

Schernhammer, E. S., Kroenke, C. H., Laden, F., and Hankinson, S. E. (2006). Night work and risk of breast cancer. Epidemiology 17, 108-111.

Schernhammer, E. S., Laden, F., Speizer, F. E., Willett, W. C., Hunter, D. J., Kawachi, I., et al. (2001). Rotating night shifts and risk of breast cancer in women participating in the nurses' health study. J. Natl. Cancer Inst. 93, 1563-1568. doi: $10.1093 /$ jnci/93.20.1563

Schernhammer, E. S., Laden, F., Speizer, F. E., Willett, W. C., Hunter, D. J., Kawachi, I., et al. (2003). Night-shift work and risk of colorectal cancer in the nurses' health study. J. Natl. Cancer Inst. 95, 825-828. doi: 10.1093/jnci/95.11.825

Semm, P., Schneider, T., and Vollrath, L. (1981). Morphological and electrophysiological evidence for habenular influence on the guinea-pig pineal gland. J. Neural Transm. 50, 247-266. doi: 10.1007/bf01249146

Shiotani, Y., Yamano, M., Shiosaka, S., Emson, P. C., Hillyard, C. J., Girgis, S., et al. (1986). Distribution and origins of substance-p (sp)-containing, calcitonin gene-related peptide (cgrp)-containing, vasoactive intestinal polypeptide (vip)containing and neuropeptide-y (npy)-containing nerve-fibers in the pinealgland of gerbils. Neurosci. Lett. 70, 187-192. doi: 10.1016/0304-3940(86) 90461-1

Shukla, M., Htoo, H. H., Wintachai, P., Hernandez, J.-F., Dubois, C., Postina, R., et al. (2015). Melatonin stimulates the nonamyloidogenic processing of $\beta$ APP through the positive transcriptional regulation of ADAM10 and ADAM17. J. Pineal Res. 58, 151-165. doi: 10.1111/jpi.12200

Sompol, P., Liu, X., Baba, K., Paul, K. N., Tosini, G., Iuvone, P. M., et al. (2011). $\mathrm{N}$-acetylserotonin promotes hippocampal neuroprogenitor cell proliferation in sleep-deprived mice. Proc. Natl. Acad. Sci. U.S.A. 108, 8844-8849. doi: 10.1073/ pnas. 1105114108

Sotthibundhu, A., Phansuwan-Pujito, P., and Govitrapong, P. (2010). Melatonin increases proliferation of cultured neural stem cells obtained from adult mouse subventricular zone. J. Pineal Res. 49, 291-300. doi: 10.1111/j.1600-079X.2010. 00794.x

Spence, D. W., Kayumov, L., Chen, A., Lowe, A., Jain, U., Katzman, M. A., et al. (2004). Acupuncture increases nocturnal melatonin secretion and reduces insomnia and anxiety: a preliminary report. J. Neuropsychiatry Clin. Neurosci. 16, 19-28. doi: 10.1176/jnp.16.1.19

Stehle, J., Reuss, S., and Vollrath, L. (1987). Electrophysiological characterization of the pineal gland of golden hamsters. Exp. Brain Res. 67, 27-32. doi: 10.1007/ bf00269449

Stener-Victorin, E., Fujisawa, S., and Kurosawa, M. (2006). Ovarian blood flow responses to electroacupuncture stimulation depend on estrous cycle and on site and frequency of stimulation in anesthetized rats. J. Appl. Physiol. (1985) 101, 84-91. doi: 10.1152/japplphysiol.01593.2005

Su, D., Chen, H., Hu, W., Liu, Y., Wang, Z., Wang, X., et al. (2018). Frequencydependent effects of subthalamic deep brain stimulation on motor symptoms in Parkinson's disease: a meta-analysis of controlled trials. Sci. Rep. 8:14456.

Takahashi, Y., and Nakajima, Y. (1996). Dermatomes in the rat limbs as determined by antidromic stimulation of sensory C-fibers in spinal nerves. Pain 67, 197202. doi: 10.1016/0304-3959(96)03116-8

Takeuchi, N., Chuma, T., Matsuo, Y., Watanabe, I., and Ikoma, K. (2005). Repetitive transcranial magnetic stimulation of contralesional primary motor 
cortex improves hand function after stroke. Stroke 36, 2681-2686. doi: 10.1161/ 01.Str.0000189658.51972.34

Tamarkin, L., Reppert, S. M., and Klein, D. C. (1979). Regulation of pineal melatonin in the Syrian hamster. Endocrinology 104, 385-389. doi: 10.1210/ endo-104-2-385

Tamarkin, L., Reppert, S. M., Klein, D. C., Pratt, B., and Goldman, B. D. (1980), Studies on the daily pattern of pineal melatonin in the Syrian hamster. Endocrinology 107, 1525-1529. doi: 10.1210/endo-107-5-1525

Tan, D. X., Xu, B., Zhou, X., and Reiter, R. J. (2018). Pineal calcification, melatonin production, aging, associated health consequences and rejuvenation of the pineal gland. Molecules 23:E301. doi: 10.3390/molecules23020301

Teclemariam-Mesbah, R., Ter Horst, G. J., Postema, F., Wortel, J., and Buijs, R. M. (1999). Anatomical demonstration of the suprachiasmatic nucleuspineal pathway. J. Compar. Neurol. 406, 171-182. doi: 10.1002/(sici)10969861(19990405)406:2<171::aid-cne3>3.0.co;2-u

Tsai, S. Y., O’Brien, T. E., and McNulty, J. A. (2001). Microglia play a role in mediating the effects of cytokines on the structure and function of the rat pineal gland. Cell Tissue Res. 303, 423-431. doi: 10.1007/s004410000330

Vanneste, S., and De Ridder, D. (2011). Bifrontal transcranial direct current stimulation modulates tinnitus intensity and tinnitus-distress-related brain activity. Eur. J. Neurosci. 34, 605-614. doi: 10.1111/j.1460-9568.2011.07778.x

Vanneste, S., Plazier, M., Ost, J., van der Loo, E., Van de Heyning, P., and De Ridder, D. (2010). Bilateral dorsolateral prefrontal cortex modulation for tinnitus by transcranial direct current stimulation: a preliminary clinical study. Exp. Brain Res. 202, 779-785. doi: 10.1007/s00221-010-2183-9

Vanneste, S., Walsh, V., Van De Heyning, P., and De Ridder, D. (2013). Comparing immediate transient tinnitus suppression using tACS and tDCS: a placebo-controlled study. Exp. Brain Res. 226, 25-31. doi: 10.1007/s00221-0133406-7

Villela, D., Atherino, V. F., Lima, L. D. S., Moutinho, A. A., do Amaral, F. G., Peres, R., et al. (2013). Modulation of pineal melatonin synthesis by glutamate involves paracrine interactions between pinealocytes and astrocytes through NF- $\mathrm{BB}$ activation. BioMed Res. Int. 2013, 618432-618432. doi: 10.1155/2013/618432

Vincent, B. (2018). Protective roles of melatonin against the amyloid-dependent development of Alzheimer's disease: a critical review. Pharmacol. Res. 134, 223-237. doi: 10.1016/j.phrs.2018.06.011

Viswanathan, A. N., Hankinson, S. E., and Schernhammer, E. S. (2007). Night shift work and the risk of endometrial cancer. Cancer Res. 67, 10618-10622. doi: 10.1158/0008-5472.can-07-2485

Volkman, P. H., and Heller, A. (1971). Pineal N-acetyltransferase activity: effect of sympathetic stimulation. Science 173, 839-840. doi: 10.1126/science.173. 3999.839

von Lanz, T., and Wachsmuth, W. (2003). Lanz/Wachsmuth Praktische Anatomie. Hals: Ein Lehr- und Hilfsbuch der anatomischen Grundlagen ärztlichen Handelns. Berlin: Springer.

Vrang, N., Larsen, P. J., Moller, M., and Mikkelsen, J. D. (1995). Topographical organization of the rat suprachiasmatic-paraventricular projection. J. Compar. Neurol. 353, 585-603. doi: 10.1002/cne.903530409

Wade, A. G., Ford, I., Crawford, G., McConnachie, A., Nir, T., Laudon, M., et al. (2010). Nightly treatment of primary insomnia with prolonged release melatonin for 6 months: a randomized placebo controlled trial on age and endogenous melatonin as predictors of efficacy and safety. BMC Med. 8:51. doi: 10.1186/1741-7015-8-51

Waldhauser, F., Waldhauser, M., Lieberman, H. R., Deng, M. H., Lynch, H. J., and Wurtman, R. J. (1984). Bioavailability of oral melatonin in humans. Neuroendocrinology 39, 307-313. doi: 10.1159/000123997

Waldhauser, F., Weiszenbacher, G., Tatzer, E., Gisinger, B., Waldhauser, M., Schemper, M., et al. (1988). Alterations in nocturnal serum melatonin levels in humans with growth and aging. J. Clin. Endocrinol. Metab. 66, 648-652. doi: 10.1210/jcem-66-3-648

Wang, S. X., Zhai, X., Li, S. Y., McCabe, M. F., Wang, X., and Rong, P. J. (2015). Transcutaneous vagus nerve stimulation induces tidal melatonin secretion and has an antidiabetic effect in zucker fatty rats. PLOS ONE 10:12. doi: 10.1371/ journal.pone.0124195

Wang, X., Figueroa Bryan, E., Stavrovskaya Irina, G., Zhang, Y., Sirianni Ana, C., Zhu, S., et al. (2009). Methazolamide and melatonin inhibit mitochondrial cytochrome C Release and are neuroprotective in experimental models of ischemic injury. Stroke 40, 1877-1885. doi: 10.1161/STROKEAHA.108.540765
Wang, Z., Wu, L., You, W., Ji, C., and Chen, G. (2013). Melatonin alleviates secondary brain damage and neurobehavioral dysfunction after experimental subarachnoid hemorrhage: possible involvement of TLR4mediated inflammatory pathway. J. Pineal Res. 55, 399-408. doi: 10.1111/jpi. 12087

Wetterberg, L. (1979). Clinical importance of melatonin. Prog. Brain Res. 52, 539-547. doi: 10.1016/s0079-6123(08)62962-3

Wong, C. S., Jow, G. M., Kaizaki, A., Fan, L. W., and Tien, L. T. (2014). Melatonin ameliorates brain injury induced by systemic lipopolysaccharide in neonatal rats. Neuroscience 267, 147-156. doi: 10.1016/j.neuroscience.2014. 02.032

Wurtman, R. J., Axelrod, J., and Phillips, L. S. (1963). Melatonin synthesis in the pineal gland: control by light. Science 142, 1071-1073.

Yamada, H., Yamamoto, A., Takahashi, M., Michibata, H., Kumon, H., and Moriyama, Y. (1996a). The L-type Ca2+ channel is involved in microvesiclemediated glutamate exocytosis from rat pinealocytes. J. Pineal Res. 21, 165-174. doi: 10.1111/j.1600-079x.1996.tb00284.x

Yamada, H., Yamamoto, A., Yodozawa, S., Kozaki, S., Takahashi, M., Morita, M., et al. (1996b). Microvesicle-mediated exocytosis of glutamate is a novel paracrine-like chemical transduction mechanism and inhibits melatonin secretion in rat pinealocytes. J. Pineal Res. 21, 175-191. doi: 10.1111/j.1600079x.1996.tb00285.x

Yamada, H., Yatsushiro, S., Ishio, S., Hayashi, M., Nishi, T., Yamamoto, A., et al. (1998). Metabotropic glutamate receptors negatively regulate melatonin synthesis in rat pinealocytes. J. Neurosci. 18, 2056-2062. doi: 10.1523/jneurosci. 18-06-02056.1998

Yamamoto, K., Matsuyama, T., Shiosaka, S., Inagaki, S., Senba, E., Shimizu, Y., et al. (1983). Overall distribution of substance-P containing nerves in the wall of the cerebral-arteries of the guinea-pig and its origins. J. Compar. Neurol. 215, 421-426. doi: 10.1002/cne.902150406

Yamashita, H., Kannan, H., Kasai, M., and Osaka, T. (1987). Decrease in blood pressure by stimulation of the rat hypothalamic paraventricular nucleus with L-glutamate or weak current. J. Auton. Nerv. Syst. 19, 229-234. doi: 10.1016/ 0165-1838(87)90069-5

Yanovski, J., Witcher, J., Adler, N., Markey, S. P., and Klein, D. C. (1987). Stimulation of the paraventricular nucleus area of the hypothalamus elevates urinary 6-hydroxymelatonin during daytime. Brain Res. Bull. 19, 129-133. doi: 10.1016/0361-9230(87)90175-4

Yatsushiro, S., Yamada, H., Hayashi, M., Yamamoto, A., and Moriyama, Y. (2000). Ionotropic glutamate receptors trigger microvesicle-mediated exocytosis of L-glutamate in rat pinealocytes. J. Neurochem. 75, 288-297. doi: 10.1046/j.14714159.2000.0750288.x

Young, I. M., Leone, R. M., Francis, P., Stovell, P., and Silman, R. E. (1985). Melatonin is metabolized to $\mathrm{N}$-acetyl serotonin and 6-hydroxymelatonin in man. J. Clin. Endocrinol. Metab. 60, 114-119. doi: 10.1210/jcem-60$1-114$

Zeitlhofer, J., Schmeiser-Rieder, A., Tribl, G., Rosenberger, A., Bolitschek, J., Kapfhammer, G., et al. (2000). Sleep and quality of life in the Austrian population. Acta Neurol. Scand. 102, 249-257.

Zemkova, H., Stojilkovic, S. S., and Klein, D. C. (2011). Norepinephrine causes a biphasic change in mammalian pinealocye membrane potential: role of alpha1B-adrenoreceptors, phospholipase C, and Ca2+. Endocrinology 152, 3842-3851. doi: 10.1210/en.2011-1180

Zempel, J. M., Zangrilli, M. A., Sutphen, C., Jerome, G., Macauley, S. L., Ju, Y.-E. S., et al. (2017). Slow wave sleep disruption increases cerebrospinal fluid amyloid- $\beta$ levels. Brain 140, 2104-2111. doi: 10.1093/brain/awx148

Zhang, H.-M., and Zhang, Y. (2014). Melatonin: a well-documented antioxidant with conditional pro-oxidant actions. J. Pineal Res. 57, 131-146. doi: 10.1111/ jpi.12162

Zhang, Y., Cook, A., Kim, J., Baranov, S. V., Jiang, J., Smith, K., et al. (2013). Melatonin inhibits the caspase-1/cytochrome c/caspase- 3 cell death pathway, inhibits MT1 receptor loss and delays disease progression in a mouse model of amyotrophic lateral sclerosis. Neurobiol. Dis. 55, 26-35. doi: 10.1016/j.nbd. 2013.03.008

Zhao, N., Zhong, C., Wang, Y., Zhao, Y., Gong, N., Zhou, G., et al. (2008). Impaired hippocampal neurogenesis is involved in cognitive dysfunction induced by thiamine deficiency at early pre-pathological lesion stage. Neurobiol. Dis. 29, 176-185. doi: 10.1016/j.nbd.2007.08.014 
Zhao, Z. Q. (2008). Neural mechanism underlying acupuncture analgesia. Prog. Neurobiol. 85, 355-375. doi: 10.1016/j.pneurobio.2008.05.004

Zhou, H., Wang, J., Jiang, J., Stavrovskaya, I. G., Li, M., Li, W., et al. (2014). $\mathrm{N}$-acetyl-serotonin offers neuroprotection through inhibiting mitochondrial death pathways and autophagic activation in experimental models of ischemic injury. J. Neurosci. 34, 2967-2978. doi: 10.1523/jneurosci.1948-13. 2014

Zisapel, N. (2018). New perspectives on the role of melatonin in human sleep, circadian rhythms and their regulation. Br. J. Pharmacol. 175, 3190-3199. doi: 10.1111/bph.14116
Conflict of Interest: The authors declare that the research was conducted in the absence of any commercial or financial relationships that could be construed as a potential conflict of interest.

Copyright $(\odot 2020$ Lumsden, Clarkson and Cakmak. This is an open-access article distributed under the terms of the Creative Commons Attribution License (CC BY). The use, distribution or reproduction in other forums is permitted, provided the original author(s) and the copyright owner(s) are credited and that the original publication in this journal is cited, in accordance with accepted academic practice. No use, distribution or reproduction is permitted which does not comply with these terms. 